\title{
Higgs-confinement phase transitions with fundamental representation matter
}

\author{
Aleksey Cherman, ${ }^{1, *}$ Theodore Jacobson ${ }^{1, \dagger}$ Srimoyee Sen, ${ }^{2, \$}$ and Laurence G. Yaffe $\oplus^{3, \S}$ \\ ${ }^{1}$ School of Physics and Astronomy, University of Minnesota, Minneapolis, Minnesota 55455, USA \\ ${ }^{2}$ Department of Physics and Astronomy, Iowa State University, Ames, Iowa 50011, USA \\ ${ }^{3}$ Department of Physics, University of Washington, Seattle, Washington 98195-1560, USA
}

(Received 23 July 2020; accepted 5 November 2020; published 24 November 2020)

\begin{abstract}
We discuss the conditions under which Higgs and confining regimes in gauge theories with fundamental representation matter fields can be sharply distinguished. It is widely believed that these regimes are smoothly connected unless they are distinguished by the realization of global symmetries. However, we show that when a $U(1)$ global symmetry is spontaneously broken in both the confining and Higgs regimes, the two phases can be separated by a phase boundary. The phase transition between the two regimes may be detected by a novel topological vortex order parameter. We first illustrate these ideas by explicit calculations in gauge theories in three spacetime dimensions. Then we show how our analysis generalizes to four dimensions, where it implies that nuclear matter and quark matter are sharply distinct phases of QCD with an approximate $S U(3)$ flavor symmetry.
\end{abstract}

DOI: 10.1103/PhysRevD.102.105021

\section{INTRODUCTION}

In gauge theories with fundamental representation matter fields, one can often dial parameters in a manner which smoothly interpolates between a Higgs regime and a confining regime without undergoing any change in the realization of global symmetries [1-4]. In the Higgs regime gauge fields become massive via the usual Higgs phenomenon, while in the confining regime gauge fields also become gapped (or acquire a finite correlation length) due to the nonperturbative physics of confinement, with an approximately linear potential appearing between heavy fundamental test charges over a finite range of length scales which is limited by the lightest meson mass. In this paper we examine situations in which the Higgs and confining regimes of such theories can be sharply distinguished.

This is, of course, an old and much-studied issue. In specific examples, when both regimes have identical realizations of global symmetries, it has been shown that confining and Higgs regimes can be smoothly connected

\footnotetext{
*acherman@umn.edu

jaco2585@umn.edu

*srimoyee08@gmail.com

§yaffe@phys.washington.edu
}

Published by the American Physical Society under the terms of the Creative Commons Attribution 4.0 International license. Further distribution of this work must maintain attribution to the author(s) and the published article's title, journal citation, and DOI. Funded by SCOAP. with no intervening phase transitions $[3,4]$. These examples, which we will refer to as the "Fradkin-ShenkerBanks-Rabinovici theorem," have inspired a widely held expectation that there can be no useful gauge-invariant order parameter distinguishing Higgs and confining phases in any gauge theory with fundamental representation matter fields. ${ }^{1}$ But there are physically interesting situations in which the Fradkin-Shenker-Banks-Rabinovici theorem does not apply. We are interested in systems where no local order parameter can distinguish Higgs and confining regimes, and yet the conventional wisdom just described is incorrect. We will analyze model theories, motivated by the physics of dense QCD, where Higgs and confining regimes cannot be distinguished by the realization of global symmetries, and yet these are sharply distinct phases necessarily separated by a quantum phase transition in the parameter space of the theory.

We will consider a class of gauge theories with two key features. The first is that they have fundamental representation scalar fields which are charged under a $U(1)$ global symmetry. Second, this $U(1)$ global symmetry is spontaneously broken in both the Higgs and confining regimes of interest. In this class of gauge theories, we argue that one can define a natural nonlocal order parameter which does distinguish the Higgs and confinement regimes. This order

\footnotetext{
${ }^{1}$ By a useful order parameter we mean an expectation value of a physical observable whose nonanalytic change also indicates nonanalytic behavior in thermodynamic observables and correlation functions of local operators. For a rather different take on these issues, see Refs. [5-8].
} 
parameter is essentially the phase of the expectation value of the holonomy (Wilson loop) of the gauge field around $U(1)$ global vortices; its precise definition is discussed below. We will find that this vortex holonomy phase acts as a topological observable; it is constant within each regime but has differing quantized values in the two regimes. ${ }^{2}$ We present a general argument-verifying it by explicit calculation where possible- that implies that nonanalyticity in our vortex holonomy observable signals a genuine phase transition separating the $U(1)$-broken Higgs and $U(1)$-broken confining regimes.

The Higgs-confinement transition we discuss in this paper does not map cleanly onto the classification of topological orders which is much discussed in modern condensed matter physics [9-12]. The basic reason is that the topological order classification is designed for gapped phases of matter, while here we focus on gapless phases. Some generalizations of topological order to gapless systems have been considered in the condensed matter literature (see e.g., Refs. [13-15]), but these examples differ in essential ways from the class of models we consider here. Our arguments also do not cleanly map onto the related idea of classifying phases based on realizations of higher-form global symmetries [16-22], because the models we consider do not have any obvious higher-form symmetries. But there is no reason to think that existing classification ideas can detect all possible phase transitions. We argue that our vortex order parameter provides a new and useful way to detect certain phase transitions which are not amenable to standard methods.

Let us pause to explain in a bit more detail why the Fradkin-Shenker-Banks-Rabinovici theorem does not apply to theories of the sort we consider. The FradkinShenker-Banks-Rabinovici theorem presupposes that Higgs fields are uncharged under any global symmetry. This assumption may seem innocuous. After all, if Higgs fields are charged under a global symmetry, it is tempting to think that this global symmetry will be spontaneously broken when the Higgs fields develop an expectation value, implying a phase transition associated with a change in symmetry realization and detectable with a local order parameter. In other words, a typical case lying within the Landau paradigm of phase transitions.

But such a connection between Higgs-confinement transitions and a change in global symmetry realization is model dependent. In the theories we consider in this paper, as well as in dense QCD, these two phenomena are unrelated. Our scalar fields will carry a global $U(1)$ charge, but crucially, the realization of all global symmetries will be the same in the confining and Higgs regimes of interest.

\footnotetext{
${ }^{2}$ This statement assumes a certain global flavor symmetry. In the absence of such a symmetry, the phase of the vortex holonomy is constant in the $U(1)$-broken confining regime and changes nonanalytically at the onset of the Higgs regime.
}

Consequently, the Fradkin-Shenker-Banks-Rabinovici theorem does not apply to these models and yet the confining and Higgs regimes are not distinguishable within the Landau classification of phases. Nevertheless, we will see that they are distinct.

The basic ideas motivating this paper were introduced by three of us in an earlier study of cold dense QCD matter [23]. We return to this motivation at the end of this paper in Sec. IV, where we generalize our analysis to cover nonAbelian gauge theories in four spacetime dimensions and explain why it provides compelling evidence against the Schäfer-Wilczek conjecture of quark-hadron continuity in dense QCD [24]. The bulk of our discussion is focused on a simpler set of model theories which will prove useful to refine our understanding of Higgs-confinement phase transitions.

We begin, in Sec. II, by introducing a simple Abelian gauge theory in three spacetime dimensions in which Higgs and confinement physics can be studied very explicitly. In Sec. III we introduce our vortex order parameter and use it to infer the existence of a Higgs-confinement phase transition. Section IV discusses the application of our ideas to four-dimensional gauge theories such as QCD, while Sec. V contains some concluding remarks. Finally, in Appendixes $\mathrm{A}-\mathrm{C}$ we collect some technical results on vortices, discuss embedding our Abelian model within a non-Abelian theory, and consider the consequences of gauging of our $U(1)$ global symmetry to produce a $U(1) \times$ $U(1)$ gauge theory.

\section{THE MODEL}

We consider compact $U(1)$ gauge theory in three Euclidean spacetime dimensions. Let $A_{\mu}$ denote the (real) gauge field. Our analysis assumes $S O(3)$ Euclidean rotation symmetry, together with a parity (or time-reversal) symmetry. Parity symmetry precludes a Chern-Simons term, so the gauge part of the action is just a photon kinetic term,

$$
S_{\gamma}=\int d^{3} x \frac{1}{4 e^{2}} F_{\mu \nu} F^{\mu \nu}
$$

The statement that the gauge group is compact (in this continuum description) amounts to saying that the Abelian description (2.1) is valid below some scale $\Lambda_{\mathrm{UV}}$, and that the UV completion of the theory above this scale allows finite action monopole-instanton field configurations whose total magnetic flux is quantized [25]. Specifically, we demand that the flux through any 2 -sphere is an integer,

$$
\int_{S^{2}} F=2 \pi k, \quad k \in \mathbb{Z}
$$

where $F \equiv \frac{1}{2} F_{\mu \nu} d x^{\mu} \wedge d x^{\nu}$ is the 2-form field strength. Condition (2.2) implies charge quantization and removes 
the freedom to perform arbitrary field rescalings of the form $A \rightarrow A^{\prime} \equiv\left(q^{\prime} / q\right) A$. As shown by Polyakov, the presence of monopole-instantons, regardless of how dilute, leads to confinement on sufficiently large distance scales [25].

\section{A. Action and symmetries}

We choose the matter sector of our model to be comprised of two oppositely-charged scalar fields, $\phi_{+}$ and $\phi_{-}$, plus one neutral scalar $\phi_{0}$. We assign unit gauge charges $q= \pm 1$ to the charged fields, making them analogous to fundamental representation matter fields in a non-Abelian gauge theory. ${ }^{3}$ We require the theory to have a single zero-form global $U(1)$ symmetry ${ }^{4}$ under which the fields $\phi_{ \pm}$both have charge assignments of -1 while $\phi_{0}$ has a charge assignment of +2 . These charge assignments, summarized here

\begin{tabular}{l|ccc} 
& $\phi_{+}$ & $\phi_{-}$ & $\phi_{0}$ \\
\hline$U(1)_{\text {gauge }}$ & +1 & -1 & 0 \\
$U(1)_{\text {global }}$ & -1 & -1 & +2
\end{tabular}

are chosen in a manner which will allow independent control of the Higgsing of the $U(1)$ gauge symmetry (or lack thereof) and the realization of the $U(1)$ global symmetry by adjusting suitable mass parameters. This is the essential structure needed to examine the issues motivating this paper in the context of a model Abelian theory.

The complete action of our model consists of the gauge action (2.1), standard scalar kinetic terms, plus a scalar potential containing interactions consistent with the above symmetries,

$$
\begin{aligned}
S= & \int d^{3} x\left[\frac{1}{4 e^{2}} F_{\mu \nu}^{2}+\left|D_{\mu} \phi_{+}\right|^{2}+\left|D_{\mu} \phi_{-}\right|^{2}\right. \\
& +m_{c}^{2}\left(\left|\phi_{+}\right|^{2}+\left|\phi_{-}\right|^{2}\right)+\left|\partial_{\mu} \phi_{0}\right|^{2}+m_{0}^{2}\left|\phi_{0}\right|^{2} \\
& -\epsilon\left(\phi_{+} \phi_{-} \phi_{0}+\text { H.c. }\right)+\lambda_{c}\left(\left|\phi_{+}\right|^{4}+\left|\phi_{-}\right|^{4}\right)+\lambda_{0}\left|\phi_{0}\right|^{4} \\
& \left.+g_{c}\left(\left|\phi_{+}\right|^{6}+\left|\phi_{-}\right|^{6}\right)+g_{0}\left|\phi_{0}\right|^{6}+\cdots+V_{\mathrm{m}}(\sigma)\right] .
\end{aligned}
$$

The mass dimensions of the various couplings are $\left[e^{2}\right]=\left[\lambda_{c}\right]=\left[\lambda_{0}\right]=1, \quad[\epsilon]=3 / 2$, and $\left[g_{c}\right]=\left[g_{0}\right]=0$.

\footnotetext{
${ }^{3}$ The fact that our charged matter fields have minimal charges of \pm 1 is an essential difference from a similar model studied by Sachdev and Park [13] in a condensed matter context (see also [15]). The model of Ref. [13] has a $U(1)$ global symmetry and fields with charges -1 and +2 under an emergent $U(1)$ gauge symmetry. The existence of nonminimally charged matter fields allowed Sachdev and Park to use topological order ideas to delineate distinct phases. That approach does not work in our model.

${ }^{4} \mathrm{~A}$ zero-form global symmetry is just an ordinary global symmetry which acts on local operators.
}

The ellipsis $(\cdots)$ represents possible further scalar selfinteractions, consistent with the imposed symmetries, arising via renormalization. The term $V_{\mathrm{m}}(\sigma)$ describes the effects of monopole instantons and is given explicitly below.

The cubic term $\epsilon \phi_{+} \phi_{-} \phi_{0}$ ensures that the model has a single $U(1)$ global symmetry, not multiple independent phase rotation symmetries. From here onward, we will denote the $U(1)$ global symmetry by $U(1)_{\mathrm{G}}$. The simplest local order parameter for the $U(1)_{\mathrm{G}}$ symmetry is just the neutral field expectation value $\left\langle\phi_{0}\right\rangle$. This order parameter has a charge assignment (2.3) of +2 under the $U(1)_{\mathrm{G}}$ symmetry; there are no gauge invariant local order parameters with odd $U(1)_{\mathrm{G}}$ charge assignments.

In addition to the $U(1)$ gauge redundancy and the $U(1)_{\mathrm{G}}$ global symmetry, this model has two internal $\mathbb{Z}_{2}$ discrete symmetries. One is a conventional (particle $\leftrightarrow$ antiparticle) charge conjugation symmetry,

$$
\left(\mathbb{Z}_{2}\right)_{\mathrm{C}}: \phi_{ \pm} \rightarrow \phi_{ \pm}^{*}, \quad \phi_{0} \rightarrow \phi_{0}^{*}, \quad A_{\mu} \rightarrow-A_{\mu}
$$

The other is a charged field permutation symmetry,

$$
\left(\mathbb{Z}_{2}\right)_{\mathrm{F}}: \phi_{+} \leftrightarrow \phi_{-}, \quad A_{\mu} \rightarrow-A_{\mu} .
$$

A conserved current $j_{\text {mag }}^{\mu} \equiv \epsilon^{\mu \nu \lambda} F_{\nu \lambda}$ associated with a $U(1)$ magnetic global symmetry is also present if monopole-instanton effects are neglected. But for our compact Abelian theory this symmetry is not present. The functional integral representation of the theory includes a sum over finite-action magnetic monopole-instanton configurations with all integer values of total magnetic charge. These induce corrections to the effective potential (below the scale $\Lambda_{\mathrm{UV}}$ ) of the form [25]

$$
V_{\mathrm{m}}(\sigma)=-\mu_{\mathrm{UV}}^{3} e^{-S_{\mathrm{I}}} \cos (\sigma) .
$$

Here $S_{\mathrm{I}}$ is the minimal action of a monopole instanton, and $\sigma$ is the dual photon field, related to the original gauge field by the Abelian duality relation ${ }^{5}$

$$
F_{\mu \nu}=\frac{i e^{2}}{2 \pi} \epsilon_{\mu \nu \lambda} \partial^{\lambda} \sigma
$$

With this normalization the dual photon field is a periodic scalar, $\sigma \equiv \sigma+2 \pi$, with the Maxwell action becoming the kinetic term $\frac{1}{2}\left(\frac{e}{2 \pi}\right)^{2}(\partial \sigma)^{2}$. The parameter $\mu_{\mathrm{UV}}$ is a shortdistance scale associated with the inverse core size of

\footnotetext{
${ }^{5}$ Expression (2.7) relies on a dilute gas approximation, valid when the instanton action is large, $S_{\mathrm{I}} \gg 1$. The duality relation (2.8) appears when one imposes the Bianchi identity for $F_{\mu \nu}$ by adding a Lagrange multiplier term $i \int d^{3} x \frac{\sigma}{4 \pi} \epsilon^{\mu \nu \lambda} \partial_{\mu} F_{\nu \lambda}$ to the Euclidean action. Relation (2.8) is the resulting equation of motion for $F_{\mu \nu}$, and integrating out $F_{\mu \nu}$ gives the Abelian dual representation of Maxwell theory.
} 
monopole-instantons. The $U(1)$ magnetic transformations act as arbitrary shifts on the dual photon field, $\sigma \rightarrow \sigma+c$. Such shifts are clearly not a symmetry, except for integer multiples of $2 \pi$. Consequently, the $U(1)_{\mathrm{G}}$ phase rotation symmetry is the only continuous global symmetry in our model.

In summary, the faithfully acting internal global symmetry group of our model is

$$
G_{\text {internal }}=\frac{\left[U(1)_{\mathrm{G}} \rtimes\left(\mathbb{Z}_{2}\right)_{\mathrm{C}}\right] \times\left(\mathbb{Z}_{2}\right)_{\mathrm{F}}}{\mathbb{Z}_{2}} .
$$

The quotient by $\mathbb{Z}_{2} \subset U(1)_{\mathrm{G}}: \phi_{ \pm} \rightarrow-\phi_{ \pm}$is necessary because it also lies in the gauge group $U(1)$.

When the charged scalar mass squared, $m_{c}^{2}$, is sufficiently negative, this theory has a Higgs regime in which the charged scalar fields are "condensed." In this regime gauge field fluctuations are suppressed since the photon acquires a mass term,

$$
\left(\left|\left\langle\phi_{+}\right\rangle\right|^{2}+\left|\left\langle\phi_{-}\right\rangle\right|^{2}\right) A_{\mu} A^{\mu} \equiv \frac{m_{A}^{2}}{2 e^{2}} A_{\mu} A^{\mu}
$$

(to lowest order in unitary gauge). ${ }^{6}$ Monopole-instantonantimonopole-instanton pairs become bound by flux tubes with a positive action per unit length $T_{\mathrm{mag}}{ }^{7}$.

In contrast, for sufficiently positive $m_{c}^{2}$ our model should be regarded as a confining gauge theory. Recall that in the context of QCD, the confining regime is characterized by a static test quark-antiquark potential which rises linearly with separation, $V_{q \bar{q}} \sim \sigma r$, for separations large compared to the strong scale, $r \gg \Lambda_{\mathrm{QCD}}^{-1}$. But such a linear potential is only present for separations where the confining string cannot break, which requires that $\sigma r<2 m_{q}$, with $m_{q}$ the mass of dynamical quarks. So confinement is only a sharply defined criterion in the heavy quark limit, $m_{q} \gg$ $\sigma / \Lambda_{\mathrm{QCD}}=\mathcal{O}\left(\Lambda_{\mathrm{QCD}}\right)$. Nevertheless, it is conventional to speak of QCD as a confining theory even with light quarks, as this is a qualitatively useful picture of the relevant dynamics. This summary applies verbatim to our compact $U(1)$ three-dimensional (3D) gauge theory with massive unit-charge matter, with $\Lambda_{\mathrm{QCD}}$ replaced by an appropriate nonperturbative scale which depends (exponentially) on the monopole-instanton action $S_{\text {I }}$ [25].

\footnotetext{
${ }^{6}$ Our charged scalar fields may be viewed as analogs of the electron pair condensate in a Ginsburg-Landau treatment of superconductivity, in which case $m_{A}$ is the Meissner mass whose inverse gives the penetration length of magnetic fields.

${ }^{7}$ On sufficiently long length scales when the flux tube length $L \gtrsim 2 S_{\mathrm{I}} / T_{\mathrm{mag}}$, these magnetic flux tubes can break due to production of monopole-instanton-antimonopole-instanton pairs. This is completely analogous to the situation in the confining regime, discussed next, where electric flux tubes exist over a limited range of scales controlled by the mass of fundamental dynamical charges.
}

Finally, we note that in the absence of monopoleinstanton effects, oppositely charged static test particles in 3D Abelian gauge theory would experience logarithmic Coulomb interactions which grow without bound with increasing separation. Such a phase could be termed "confined," but for our purposes this terminology is not helpful. We find it more appropriate to reserve the term "confinement" for situations where the potential between test charges is linear over a significant range of distance scales. With this terminology, 3D compact $U(1)$ gauge theory with finite-action monopole instantons and very heavy charged matter is confining, while the noncompact version of the theory, which does not have a regime with a linear potential between test charges, is not confining.

\section{B. Analogy to dense QCD}

Our 3D Abelian model is designed to mimic many features of real 4D QCD at nonzero density. Explicitly,

(1) Both theories contain fundamental representation matter fields and are confining in the sense described above. Of course, the gauge groups are completely different: $S U(N)$ versus $U(1)$.

(2) QCD with massive quarks of equal mass has a vectorlike $U\left(N_{f}\right) / \mathbb{Z}_{N}$ internal global symmetry. The quotient arises because $\mathbb{Z}_{N}$ transformations are part of the $S U(N)$ gauge symmetry. In our model, the corresponding global symmetry is $\left[\left(\mathbb{Z}_{2}\right)_{\mathrm{F}} \times U(1)_{\mathrm{G}}\right] / \mathbb{Z}_{2}$. The $\left(\mathbb{Z}_{2}\right)_{\mathrm{F}} \times U(1)_{\mathrm{G}}$ symmetry is analogous to $U\left(N_{f}\right)$, while the discrete quotient arises for the same reason as in QCD.

(3) The scalar fields in the 3D Abelian model may be regarded as playing the role of color antifundamental diquark operators which acquire nonzero vacuum expectation values in high density QCD (see Ref. [26] for a review). The symmetry group $U(1)_{\mathrm{G}}$ is analogous to quark number $U(1) \subset$ $U\left(N_{f}\right)$, while $U(1)_{\mathrm{G}} / \mathbb{Z}_{2}$ is analogous to baryon number $U(1)_{B}$. Note one distinction in the transformation properties of the scalar fields in our Abelian model and the diquark condensates in QCD; the former have charge 1 under our $U(1)_{\mathrm{G}}$ group, whereas the latter have charge 2 under the quark number. The $\left(\mathbb{Z}_{2}\right)_{\mathrm{F}}$ permutation symmetry of our 3D Abelian model is analogous to the $\mathbb{Z}_{N_{f}} \subset$ $U\left(N_{f}\right)$ cyclic flavor permutation symmetry of 4D QCD.

(4) Since the charged scalars $\phi_{ \pm}$are analogous to antifundamental diquarks in three-color $\mathrm{QCD}$, $\phi_{+}^{\dagger} \phi_{-}^{\dagger}$ is akin to a dibaryon. This means that $\phi_{0}$ can also be interpreted as a dibaryon interpolating operator, and the condensation of $\phi_{0}$ in our model is directly analogous to the dibaryon condensation which occurs in dense QCD.

(5) In QCD, the Vafa-Witten theorem [27] implies that phases with spontaneously broken $U(1)_{B}$ symmetry 


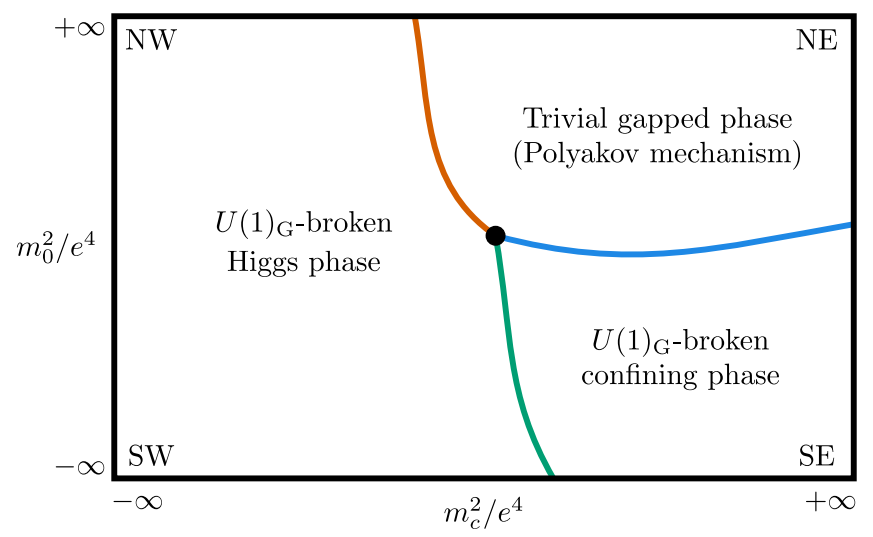

FIG. 1. A sketch of the simplest consistent phase diagram of our model as a function of the charged and neutral scalar mass parameters $m_{c}^{2}$ and $m_{0}^{2}$. The four corners correspond to weakly coupled regimes in parameter space; curves in the interior of the figure represent phase transitions. These phase transition curves are robust: they cannot be evaded by varying any parameters of the model which are consistent with its symmetries.

can only appear at nonzero baryon density, while in our Abelian model $U(1)_{\mathrm{G}}$-broken phases can appear at zero density. This difference reflects the fact that QCD contains only fermionic matter fields, while our Abelian model has fundamental scalar fields.

\section{Symmetry constraints on the phase structure}

We begin analyzing the phase structure of the model (2.4) using the Landau paradigm based on realizations of symmetries with local order parameters. We will consider the phase diagram as a function of the charged and neutral scalar masses, $m_{c}^{2}$ and $m_{0}^{2}$. We focus on the regime where quartic and sextic scalar self-couplings are positive; the cubic, quartic, and gauge couplings are comparable; $\epsilon / e^{3}$, $\left|\lambda_{c}\right| / e^{2}$, and $\left|\lambda_{0}\right| / e^{2}$ are all $\mathcal{O}(1)$; and the dimensionless sextic couplings are small, $g_{c}, g_{0} \ll 1$. The simplest phase diagram consistent with our analysis is sketched in Fig. 1.

Interpreting Fig. 1 as if it were a map, let us refer to the four weakly coupled corners of parameter space by their compass directions:

$\mathrm{NW}:\left\{-m_{c}^{2} \gg e^{4}, m_{0}^{2} \gg e^{4}\right\}, \quad \mathrm{NE}:\left\{m_{c}^{2} \gg e^{4}, m_{0}^{2} \gg e^{4}\right\}$,

$\mathrm{SW}:\left\{-m_{c}^{2} \gg e^{4},-m_{0}^{2} \gg e^{4}\right\}, \quad \mathrm{SE}:\left\{m_{c}^{2} \gg e^{4},-m_{0}^{2} \gg e^{4}\right\}$,

each of which we discuss in turn. In this section we explain the origin of the phase transition curve (orange) separating the NE region from the $\mathrm{W}$ side of Fig. 1, as well as the (blue) curve separating the NE and SE regions. The bulk of the paper is dedicated to understanding the origin of the phase transition curve (green) separating the SE region from the W side of Fig. 1.

First, consider region NE where $m_{c}^{2}, m_{0}^{2} \gg e^{4}$. In this regime our model has a unique gapped vacuum state and no broken symmetry. To see this, one may integrate out all the matter fields and observe that the resulting tree-level effective action is

$$
S_{\mathrm{eff}}=\int d^{3} x\left[\frac{1}{4 e^{2}} F_{\mu \nu}^{2}+V_{\mathrm{m}}(\sigma)\right]
$$

The monopole potential $V_{\mathrm{m}}(\sigma)$ has a unique minimum for the dual photon $\sigma$ and induces a nonzero photon mass,

$$
m_{\gamma}^{2}=4 \pi^{2}\left(\mu_{\mathrm{UV}}^{3} / e^{2}\right) e^{-S_{\mathrm{I}}}
$$

Hence, the vacuum is gapped and unique. Both the continuous $U(1)_{\mathrm{G}}$ and the discrete $\left(\mathbb{Z}_{2}\right)_{\mathrm{C}}$ and $\left(\mathbb{Z}_{2}\right)_{\mathrm{F}}$ global symmetries are unbroken, and hence region NE may be termed "confining and unbroken."

Now consider the entire E side where $m_{c}^{2} \gg e^{4}$ while the neutral mass $m_{0}^{2}$ is arbitrary. Then one may integrate out the charged fields and the effective action becomes

$$
\begin{aligned}
S_{\mathrm{eff}}= & \int d^{3} x\left[\frac{1}{4 e^{2}} F_{\mu \nu}^{2}+V_{\mathrm{m}}(\sigma)+\left|\partial_{\mu} \phi_{0}\right|^{2}+m_{0}^{2}\left|\phi_{0}\right|^{2}\right. \\
& \left.+\lambda_{0}\left|\phi_{0}\right|^{4}+g_{0}|\phi|^{6}+\cdots\right] .
\end{aligned}
$$

This is a 3D $X Y$ model plus a decoupled compact $U(1)$ gauge theory. The photon is still gapped by the Polyakov mechanism. If we take $m_{0}^{2} \gg\left|\lambda_{0}\right|^{2}$, then we come back to the discussion of the previous paragraph. If we take $-m_{0}^{2} \gg\left|\lambda_{0}\right|^{2}$, then $\phi_{0}$ develops a nonvanishing expectation value, the $U(1)_{\mathrm{G}} / \mathbb{Z}_{2}$ symmetry is spontaneously broken, and there is a single massless Nambu-Goldstone boson. So region SE is "confining and $U(1)_{\mathrm{G}}$ symmetry broken." The discrete $\left(\mathbb{Z}_{2}\right)_{\mathrm{F}}$ symmetry is unbroken in this region, as is a redefined $\left(\mathbb{Z}_{2}\right)_{\mathrm{C}}$ symmetry which combines the basic $\left(\mathbb{Z}_{2}\right)_{\mathrm{C}}$ transformation (2.5) with a $U(1)_{\mathrm{G}}$ transformation that compensates for the arbitrary phase of the condensate $\left\langle\phi_{0}\right\rangle$. This symmetry-broken regime must be separated from the symmetry-unbroken regime by a phase transition depending on the value of $m_{0}^{2} / \lambda_{0}^{2}$. If we take our quartic and sextic couplings to be positive, this is just the well-known $X Y$ model phase transition, which is second order in three spacetime dimensions.

Next, consider what happens on the $\mathrm{W}$ side where $-m_{c}^{2} \gg e^{4}$ while $m_{0}^{2}$ is arbitrary. In this case the charged scalar fields $\phi_{ \pm}$will acquire nonzero expectation 
values (using gauge-variant language), with $v_{c} \equiv\left|\left\langle\phi_{ \pm}\right\rangle\right|=$ $\mathcal{O}\left(\left|m_{c} \lambda_{c}^{-1 / 2}\right|\right){ }^{8}$

This has several effects. First, since these fields transform nontrivially under the $U(1)_{\mathrm{G}}$ symmetry, this global symmetry is spontaneously broken leading to a massless Nambu-Goldstone excitation. Second, the $U(1)$ gauge field becomes Higgsed, as discussed above, with the photon acquiring a mass $m_{A}$. Writing $\phi_{ \pm}=\left(v_{c}+H_{ \pm} / \sqrt{2}\right) e^{-i \chi}$, up to an arbitrary $U(1)$ gauge transformation, the resulting effective action has the form

$$
\begin{aligned}
S= & \int d^{3} x\left[\frac{1}{4 e^{2}} F_{\mu \nu}^{2}+\frac{1}{2} m_{A}^{2} A_{\mu} A^{\mu}+\left|\partial_{\mu} \phi_{0}\right|^{2}\right. \\
& +m_{0}^{2}\left|\phi_{0}\right|^{2}+\lambda_{0}\left|\phi_{0}\right|^{4}+2 v_{c}^{2}\left(\partial_{\mu} \chi\right)^{2}-2 \epsilon v_{c}^{2} \operatorname{Re}\left(e^{-2 i \chi} \phi_{0}\right) \\
& \left.+\sum_{i= \pm}\left[\frac{1}{2}\left(\partial_{\mu} H_{i}\right)^{2}+\frac{1}{2} m_{H}^{2} H_{i}^{2}\right]+\cdots\right],
\end{aligned}
$$

where $\chi$ is the $U(1)_{\mathrm{G}}$ Nambu-Goldstone boson and $H_{ \pm}$are real Higgs modes with mass $m_{H}$. This regime, extending inward from the $\mathrm{W}$ boundary of the phase diagram, may be termed "Higgsed and $U(1)_{\mathrm{G}}$ symmetry broken." The discrete symmetries remain unbroken in the same manner as in region SE. Regardless of the sign of $m_{0}^{2}$, the neutral scalar $\phi_{0}$ acquires a nonzero vacuum expectation value whose phase, $2 \chi$, is set by the phase of the Higgs condensate. As $m_{0}^{2}$ is varied from large positive to large negative values, the magnitude $\left|\left\langle\phi_{0}\right\rangle\right|$ varies from a small $\mathcal{O}\left(\epsilon v_{c}^{2} m_{0}^{-2}\right)$ value to a large $\mathcal{O}\left(m_{0} \lambda_{0}^{-1 / 2}\right)$ value, while always remaining nonzero. Throughout this Higgs regime monopole-instanton-antimonopole-instanton pairs become linearly confined by magnetic flux tubes as noted earlier.

The fact that the $U(1)_{\mathrm{G}}$ symmetry is spontaneously broken in this Higgs regime means that the entire $\mathrm{W}$ region of parameter space with $-m_{c}^{2} \gg e^{4}$ must be separated by a phase transition from the trivially gapped region NE where $m_{c}^{2}$ and $m_{0}^{2}$ are large and positive. But the pattern of global symmetry breaking throughout the $\mathrm{W}$ side Higgs regime of $-m_{c}^{2} \gg e^{4}$ is identical to that in region SE where $m_{c}^{2} \gg e^{4}$ and $-m_{0}^{2} \gg \lambda_{0}^{2}$. This raises the central question in this paper:

Are the Higgs and confining $U(1)_{\mathrm{G}}$-breaking regimes smoothly connected, or are they distinct phases?

As summarized in the Introduction and sketched in Fig. 1, we will find that the Higgs and confining $U(1)_{\mathrm{G}^{-}}$ breaking regimes must be distinct phases, separated by at least one phase transition, even though there are no distinguishing local order parameters.

Before leaving this section, we pause to consider two further issues: the realization of the $\left(\mathbb{Z}_{2}\right)_{\mathrm{F}}$ symmetry and the nature of the $\epsilon \rightarrow 0$ limit. In our discussion below we

\footnotetext{
${ }^{8}$ In this and subsequent parametric estimates, we neglect the cubic coupling and sextic couplings. For the sextic couplings this is justified by our assumption that they are small. We have dropped $\epsilon$ dependence purely for simplicity: taking it into account is straightforward but results in much more cumbersome expressions.
}

will assume that the $\left(\mathbb{Z}_{2}\right)_{\mathrm{F}}$ symmetry is not spontaneously broken. It is possible to tune the scalar potential to break $\left(\mathbb{Z}_{2}\right)_{\mathrm{F}}$ spontaneously, but this results in a Higgs phase which is separated by an obvious phase boundary from both the $U(1)_{\mathrm{G}}$-broken confining phase and the $\left(\mathbb{Z}_{2}\right)_{\mathrm{F}^{-}}$ invariant Higgs phase. This makes the $\left(\mathbb{Z}_{2}\right)_{\mathrm{F}}$-broken regime uninteresting for the purposes of this paper.

Next, one should observe that $\epsilon \rightarrow 0$ is a nongeneric limit of the model. An additional global symmetry which purely phase rotates the charged fields, $\phi_{ \pm} \rightarrow e^{i \alpha} \phi_{ \pm}$, is present when $\epsilon=0$; we denote this symmetry as $U(1)_{\text {extra }}$. The $\epsilon=$ 0 theory has four distinct phases distinguished by realizations of the $U(1)_{\mathrm{G}}$ and $U(1)_{\text {extra }}$ symmetries. There is a phase where only the $U(1)_{\text {extra }}$ symmetry is spontaneously broken, with one Nambu-Goldstone boson. This phase is not present at nonzero $\epsilon$. At $\epsilon=0$, the $U(1)_{\mathrm{G}}$-broken Higgs phase in Fig. 1 becomes a phase with two spontaneously broken continuous global symmetries, $U(1)_{\mathrm{G}}$ and $U(1)_{\text {extra }}$, and has two Nambu-Goldstone bosons. This is a distinct symmetry realization from the $U(1)_{\mathrm{G}}$-broken confining regime with only a single Nambu-Goldstone boson implying, by the usual Landau paradigm reasoning, at least one intervening separating phase transition.

When $\epsilon$ is nonzero but very small compared to all other scales, there is a parametrically light pseudo-NambuGoldstone boson with a mass $m_{\mathrm{pNGB}} \propto \sqrt{\epsilon}$ in the Higgs regime. Determining whether the $U(1)_{\mathrm{G}}$-broken Higgs and confining regimes remain distinct for nonzero values of $\epsilon$ is the goal of our next section in which we examine the longdistance behavior of holonomies around vortices. In this analysis, it will be important that the holonomy contour radius be large compared to microscopic length scaleswhich include the Compton wavelength of the pseudoGoldstone boson, $m_{\mathrm{pNGB}}^{-1}$. There is nonuniformity between the large distance limit of the holonomy and the $\epsilon \rightarrow 0$ limit, and consequently the physics of interest must be studied directly in the theory with $\epsilon \neq 0$.

\section{VORTICES AND HOLONOMIES}

\section{A. The order parameter $O_{\Omega}$}

Consider the portion of the phase diagram in which the $U(1)_{\mathrm{G}}$ symmetry is spontaneously broken. Then the field $\phi_{0}$ has a nonvanishing expectation value and the spectrum contains a Nambu-Goldstone boson. The Goldstone manifold has a nontrivial first homotopy group, $\pi_{1}\left(U(1)_{\mathrm{G}}\right)=\mathbb{Z}$. This implies that there are stable global vortex excitations, which are particlelike excitations in two spatial dimensions.

Vortex excitations may be labeled by an integer winding number $w$ indicating the number of times the phase of $\left\langle\phi_{0}\right\rangle$ wraps the unit circle as one encircles a vortex. More explicitly, one may write the winding number as a contour integral of the gradient of the phase,

$$
w=\frac{1}{2 \pi} \oint_{C} d x^{\mu} u_{\mu}
$$


where $u_{\mu} \equiv-i \partial_{\mu}\left(\left\langle\phi_{0}\right\rangle /\left|\left\langle\phi_{0}\right\rangle\right|\right)$. Using the language of a superfluid, $u_{\mu}$ is the superfluid flow velocity, and the winding number $w$ is the quantized circulation around a vortex.

As with vortices in superfluid films, vortex excitations have logarithmic long range interactions, with a $1 / r$ force between vortices separated by distance $r$. A single vortex in infinite space has a logarithmically divergent long distance contribution to its self-energy. Nevertheless, vortices are important collective excitations and, in any sufficiently large volume, a nonzero spatial density of vortices and antivortices will be present due to quantum and/or thermal fluctuations. From a spacetime perspective, vortex/antivortex worldlines, as they appear and annihilate, form a collection of closed loops, with an action scaling as $L \log L$ for loops with characteristic size $L .{ }^{9}$

Consider the gauge field holonomy, $\Omega \equiv e^{i \oint_{C} A}$, evaluated on some large circular contour $C$ surrounding a vortex of nonzero winding number $k$, illustrated in Fig. 2, which we denote by $\langle\Omega(C)\rangle_{k}$. Let $r$ denote the radius of the contour $C$ encircling the vortex. We are interested in the phase of the holonomy, but as the size of the contour $C$ grows, short distance quantum fluctuations will cause the magnitude of the expectation $\langle\Omega(C)\rangle_{k}$ to decrease (with at least exponential perimeter-law decrease). To compensate, we consider the large distance limit of a ratio of the holonomy expectation values which do, or do not, encircle a vortex of minimal nonzero winding number,

$$
O_{\Omega} \equiv \lim _{r \rightarrow \infty} \frac{\langle\Omega(C)\rangle_{1}}{\langle\Omega(C)\rangle}
$$

Here, the numerator should be understood as an expectation value defined by a constrained functional integral in which there is a prescribed vortex loop of characteristic size $r$ and winding number 1 linked with the holonomy loop of size $r$, with both sizes, and the minimal separation between the two loops, scaling together as $r$ increases. The denominator is the ordinary unconstrained vacuum expectation value.

The quantity $O_{\Omega}$ measures the phase acquired by a particle with unit gauge charge when it encircles a minimal global vortex. Or equivalently, it is the phase acquired by a minimal global vortex when it is dragged around a particle with unit gauge charge.

Our analysis below will demonstrate that $O_{\Omega}$ cannot be a real-analytic function of the charged scalar mass parameter $m_{c}^{2} / e^{4}$. We will also argue that nonanalyticities in the topological order parameter $O_{\Omega}$ are associated with genuine thermodynamic phase transitions.

\footnotetext{
${ }^{9}$ This is only a logarithmic enhancement over the linear scaling of a vortex loop action in superconductors (or simple Abelian Higgs models).
}

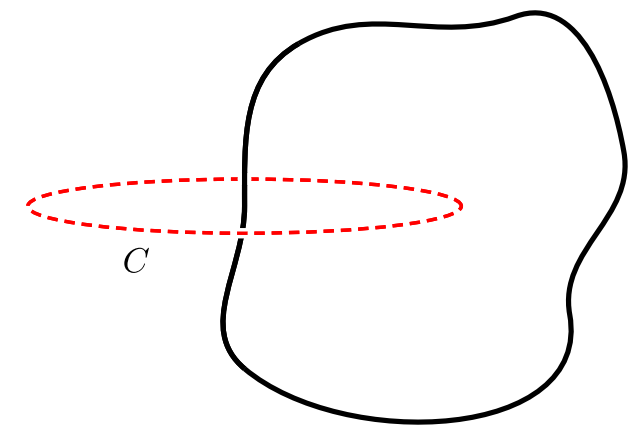

FIG. 2. A contour $C$ (red dashed curve) which links a vortex worldline (solid black curve). Of interest is the gauge field holonomy $\Omega \equiv e^{i \oint_{C} A}$ for contours $C$ far from the vortex core.

A quick sketch of the argument is as follows. Since the vacuum is invariant under the $\left(\mathbb{Z}_{2}\right)_{\mathrm{C}}$ charge conjugation symmetry, the denominator of $O_{\Omega}$ must be real and at sufficiently weak coupling is easily seen to be positive. ${ }^{10}$ In the constrained expectation value in the numerator of $O_{\Omega}$, the $\left(\mathbb{Z}_{2}\right)_{\mathrm{C}}$ symmetry is explicitly broken by the unit-circulation condition that enters the definition of $\langle\Omega(C)\rangle_{1}$. But the unit-circulation condition does not break $\left(\mathbb{Z}_{2}\right)_{\mathrm{F}}$ permutation symmetry (2.6), which also flips the sign of the gauge field. ${ }^{11}$ Therefore the numerator of $O_{\Omega}$ must be invariant under $\left(\mathbb{Z}_{2}\right)_{\mathrm{F}}$, and hence real. We will see below that it is negative deep in the Higgs regime, but is positive deep in the $U(1)_{\mathrm{G}}$-broken confining regime. In the large- $r$ limit defining our vortex observable $O_{\Omega}$, the magnitudes of the holonomy expectations in numerator and denominator will be identical. Hence, our vortex observable $O_{\Omega}$ obeys

$O_{\Omega}= \begin{cases}-1, & U(1)_{\mathrm{G}} \text {-broken Higgs regime; } \\ +1, & U(1)_{\mathrm{G}} \text {-broken confining regime, }\end{cases}$

and therefore cannot be analytic as a function of $m_{c}^{2} / e^{4}$.

In the remainder of this section we support the above claims. We study the properties of vortices in the Higgs and confining $U(1)_{\mathrm{G}}$-broken regimes in Secs. III B and III C, respectively. Then in Sec. III D we argue that

\footnotetext{
${ }^{10}$ One may equally well appeal to reflection symmetry, as this reverses the orientation of a reflection symmetric contour like a circle, and hence maps the holonomy on a circular contour to its complex conjugate. This alternative will be relevant for our later discussion in Sec. IV of dense QCD and related models with nonzero chemical potential, where charge conjugation symmetry is explicitly broken by the chemical potential but the ground state remains invariant under reflections.

${ }^{11}$ The $\left(\mathbb{Z}_{2}\right)_{\mathrm{F}}$ symmetry cannot be spontaneously broken due to the presence of a vortex because the vortex worldvolume is onedimensional, and discrete symmetries cannot break spontaneously in one spacetime dimension. (The exception to this statement involving mixed 't Hooft anomalies [28] is irrelevant in our case.)
} 
nonanalyticities in our topological order parameter are associated with genuine thermodynamic phase transitions. Finally, in Sec. III E we extend the treatment and consider the effects of perturbations which explicitly break the $\left(\mathbb{Z}_{2}\right)_{\mathrm{F}}$ symmetry. We find that $O_{\Omega}$ remains a nonanalytic function of the charged scalar mass parameter(s) even in the presence of such perturbations. This shows that the phase transition line separating the Higgs and confining $U(1)_{\mathrm{G}^{-}}$ broken regimes is robust against sufficiently small $\left(\mathbb{Z}_{2}\right)_{\mathrm{F}^{-}}$ breaking perturbations.

\section{B. $O_{\Omega}$ in the Higgs regime}

We first consider $O_{\Omega}$ deep in the Higgs regime, $-m_{c}^{2} \gg$ $e^{4}$ and, to begin, neglect quantum fluctuations altogether. So the holonomy expectation values in the definition (3.2) of $O_{\Omega}$ just require evaluation of the holonomy in the appropriate energy-minimizing classical field configurations.

As always, the holonomy $\Omega(C)$ is the exponential of the line integral $\oint_{C} A$ (times $i$ ) which, in our Abelian theory, is just the magnetic flux passing through a surface spanning the curve $C$. For the ordinary vacuum expectation value in the denominator of $O_{\Omega}$, vacuum field configurations have everywhere vanishing magnetic field and hence $\langle\Omega(C)\rangle=1$.

For the constrained expectation value in the numerator, one needs to understand the form of the minimal vortex solution(s). Choose coordinates such that the vortex lies at the origin of space and let $\{r, \theta\}$ denote 2D polar coordinates. For a vortex configuration with winding number $k$, the phase of the neutral scalar $\phi_{0}$ must wrap $k$ times around the unit circle as one encircles the origin.

There exist classical solutions which preserve rotation invariance, and we presume that these rotationally invariant solutions capture the relevant global energy minima. Such field configurations may be written in the explicit form

$$
\begin{gathered}
\phi_{+}(r, \theta)=v_{c} f_{+}(r) e^{i \nu_{+} \theta}, \quad \phi_{0}(r, \theta)=v_{0} f_{0}(r) e^{i k \theta}, \\
\phi_{-}(r, \theta)=v_{c} f_{-}(r) e^{i \nu_{-} \theta}, \quad A_{\theta}(r)=\frac{\Phi h(r)}{2 \pi r} .
\end{gathered}
$$

Here $v_{0}$ and $v_{c}$ are the magnitudes of the vacuum expectation values of $\phi_{0}$ and $\phi_{ \pm}$, determined by minimizing the potential terms in the action. The angular wave numbers $\nu_{+}, \nu_{-}$, and $k$ must be integers to have single valued configurations, and $k$, by definition, is the winding number of the vortex configuration. For nonzero values of $k$ and $\nu_{ \pm}$the radial functions $f_{0}(r)$ and $f_{ \pm}(r)$ interpolate between 0 at the origin and 1 at infinity. Similarly, to minimize energy the gauge field must approach a pure gauge form at a large distance, implying that $h(r)$ may also be taken to interpolate between 0 and 1 as $r$ goes from the origin to infinity. The associated magnetic field is

$$
B(r)=\frac{\left(r A_{\theta}(r)\right)^{\prime}}{r}=\frac{\Phi h^{\prime}(r)}{2 \pi r} .
$$

The gauge field in ansatz (3.4) is written in a form which makes the coefficient $\Phi$ equal to the total magnetic flux,

$\Phi_{B} \equiv \int d^{2} x B=2 \pi \int_{0}^{\infty} r d r B(r)=\Phi \int_{0}^{\infty} d r h^{\prime}(r)=\Phi$.

To avoid having an energy which diverges linearly with volume (relative to the vacuum), the phases of $\phi_{0}, \phi_{+}$, and $\phi_{-}$must be correlated in a fashion which minimizes the cubic term in the action. Below we will suppose that the coefficient of the cubic term $\epsilon>0$, but essentially the same formulas would result if $\epsilon<0$. (The singular point $\epsilon=0$ must be handled separately; see the discussion at the end of Sec. II C.) Minimizing the cubic term in the action forces the product $\phi_{0} \phi_{+} \phi_{-}$to be real and positive, implying that

$$
\nu_{+}=n-k, \quad \nu_{-}=-n,
$$

for some integer $n$.

After imposing condition (3.7), there remains a logarithmic dependence on the spatial volume caused by the scalar kinetic terms which, due to the angular phase variation of the scalar fields, generate energy densities falling as $1 / r^{2}$. Explicitly, this long-distance energy density is

$$
\begin{aligned}
\mathcal{E}(r)= & \frac{v_{c}^{2}}{r^{2}}\left[\left(n-k-\frac{\Phi}{2 \pi}\right)^{2}+\left(-n+\frac{\Phi}{2 \pi}\right)^{2}\right] \\
& +\frac{v_{0}^{2} k^{2}}{r^{2}}+\mathcal{O}\left(r^{-4}\right) .
\end{aligned}
$$

Minimizing this IR energy density, for given values of $k$ and $n$, determines the magnetic flux $\Phi$, leading to

$$
\Phi_{B}=\Phi=(2 n-k) \pi,
$$

and an IR energy density $\mathcal{E}(r)=\left(\frac{1}{2} v_{c}^{2}+v_{0}^{2}\right) k^{2} / r^{2}+\mathcal{O}\left(r^{-4}\right)$.

The explicit form of the radial functions is determined by minimizing the remaining IR finite contributions to the energy. These consist of the magnetic field energy and short distance corrections to the scalar field kinetic and potential terms, all of which are concentrated in the vortex core region. Semiexplicitly, 


$$
\begin{aligned}
E= & 2 \pi \int r d r\left[\frac{h^{\prime}(r)^{2}}{8 e^{2} r^{2}}(2 n-k)^{2}+\frac{v_{c}^{2} f_{+}(r)^{2}}{4 r^{2}}[(2 n-k)(1-h(r))-k]^{2}\right. \\
& +\frac{v_{0}^{2} k^{2} f_{0}(r)^{2}}{r^{2}}+\frac{v_{c}^{2} f_{-}(r)^{2}}{4 r^{2}}[(2 n-k)(1-h(r))+k]^{2} \\
& \left.+v_{c}^{2}\left[f_{+}^{\prime}(r)^{2}+f_{-}^{\prime}(r)^{2}\right]+v_{0}^{2} f_{0}^{\prime}(r)^{2}+(\text { potential terms })\right] .
\end{aligned}
$$

Minimizing this energy leads to straightforward but unsightly ordinary differential equations which determine the precise form of the radial profile functions; see Appendix A. Qualitatively, the gauge field radial function $h(r)$ approaches its asymptotic value of one exponentially fast on the length scale $\min \left(m_{A}^{-1}, \tilde{m}^{-1}\right)$, where $m_{A}=2 e v_{c}$ and $\tilde{m}^{2} \equiv 4 \lambda_{c} v_{c}^{2}+2 \epsilon v_{0}$. The scalar field profile functions $f_{0}(r)$ and $f_{ \pm}(r)$ approach their asymptotic large $r$ values with $1 / r^{2}$ corrections on the length scales set by the corresponding masses $m_{0}$ and $m_{c}$.

For a given nonzero winding number $k$, the above procedure generates an infinite sequence of vortex solutions distinguished by the value of $n$, or more physically by the quantized value of the magnetic flux (3.9) carried in the vortex core. The minimal energy vortex, for a given winding number, is the one which minimizes this flux. For even winding numbers, this is $n=k / 2$ and vanishing magnetic flux. In such solutions, the phases of the two charged scalar fields are identical with $\nu_{ \pm}=-k / 2$.

For odd winding number $k$ there are two degenerate solutions with $n=(k \pm 1) / 2$ and magnetic flux $\Phi= \pm \pi$. In these solutions, the charged scalar fields have differing phase windings with $\nu_{+}=-(k \mp 1) / 2$ and $\nu_{-}=-(k \pm 1) / 2$. For minimal $|k|=1$ vortices, one of the charged scalars has a constant phase with no winding, while the other charged scalar has a phase opposite that of $\phi_{0}$.

The gauge field holonomy surrounding a vortex, far from its core, is simply \pm 1 depending on whether the magnetic flux is an even or odd multiple of $\pi$ and this, in turn, merely depends on whether the vortex winding number $k$ is even or odd,

$$
\langle\Omega(C)\rangle_{k}=e^{i \Phi}=(-1)^{k} .
$$

The net result is that there are four different minimal energy vortex solutions, illustrated in Fig. 3, having $(k, \Phi)=(1, \pi),(1,-\pi),(-1, \pi)$, and $(-1,-\pi)$. As indicated in the figure, the $\left(\mathbb{Z}_{2}\right)_{\mathrm{F}}$ symmetry interchanges vortices with identical winding number and opposite values of magnetic flux, while the $\left(\mathbb{Z}_{2}\right)_{\mathrm{C}}$ symmetry interchanges vortices with opposite values of both winding number and magnetic flux. Therefore, all these vortices have identical energies. For our purposes, the key result is that the long distance holonomy is the same for all minimal vortices, namely $\langle\Omega(C)\rangle_{k= \pm 1}=-1$. Consequently, we find

$$
O_{\Omega}=-1 \text { at tree level. }
$$

We now consider the effects of quantum fluctuations on this result. Using standard effective field theory (EFT) reasoning, as one integrates out fluctuations below the UV scale $\Lambda_{\mathrm{UV}}$, the action (2.4) will receive scale-dependent corrections which (a) renormalize the coefficients of operators appearing in the action (2.4), and (b) induce additional operators of increasing dimension consistent with the symmetries of the theory. But the result (3.12) follows directly from the leading long-distance form (3.8) of the energy density whose minimum fixes the vortex magnetic flux equal to $\pm \pi$ for minimal winding vortices. Because this $1 / r^{2}$ energy density leads to a total energy which is logarithmically sensitive to the spatial volume, short distance IR-finite contributions to the energy cannot affect the flux quantization condition (3.9) in the limit of large spatial volume. Only those corrections which modify this $1 / r^{2}$ long distance energy density have the potential to change the quantization condition.

One may construct the long distance EFT as an expansion in derivatives, with the effective expansion parameter being the small ratio of fundamental length scales (such as the vortex core size or Compton wavelengths of massive excitations) to the arbitrarily large length scale of interest. Any term in the EFT action with more than two derivatives will produce a contribution to the energy density which

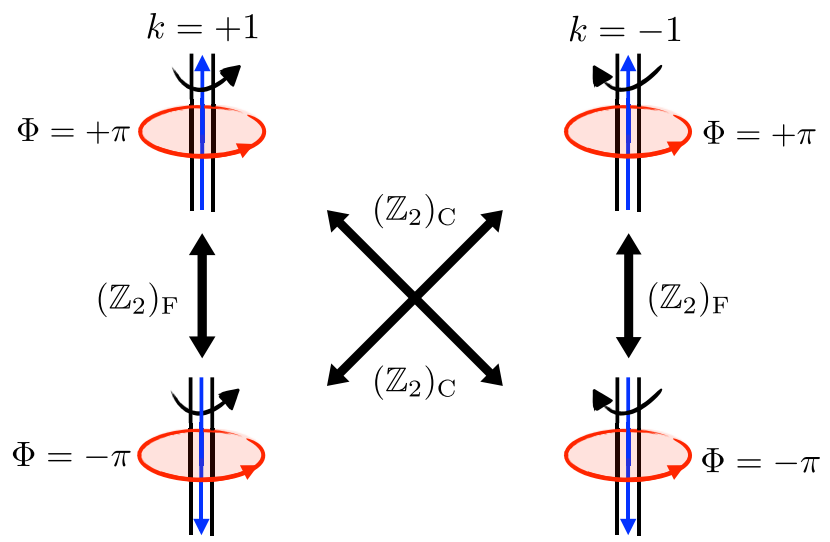

FIG. 3. There are four distinct minimal energy vortex solutions, with winding number $k= \pm 1$ and magnetic flux $\Phi= \pm \pi$. The $\left(\mathbb{Z}_{2}\right)_{\mathrm{F}}$ and $\left(\mathbb{Z}_{2}\right)_{\mathrm{C}}$ discrete symmetries relate these vortices as shown. 
falls faster than $1 / r^{2}$ when evaluated on a vortex configuration, and hence cannot contribute to the $\mathcal{O}\left(1 / r^{2}\right)$ long distance energy density (3.8). Similarly, terms with less than two derivatives also do not contribute to the $\mathcal{O}\left(1 / r^{2}\right)$ long distance energy density (3.8). Hence the only fluctuation-induced terms that might affect the long distance vortex holonomy are those with precisely two derivatives acting on the charged scalar fields. Consequently, the portion of the effective action that controls holonomy expectation values around vortices can be written in the form

$$
\begin{aligned}
S_{\text {eff }, U(1) \text { holonomy }}= & \int d^{3} x\left\{f_{1}\left(\phi_{0}, \phi_{+}, \phi_{-}\right)\left(D_{\mu} \phi_{+}\right)\left(D^{\mu} \phi_{+}\right)^{\dagger}+f_{1}\left(\phi_{0}, \phi_{-}, \phi_{+}\right)\left(D_{\mu} \phi_{-}\right)\left(D^{\mu} \phi_{-}\right)^{\dagger}\right. \\
& +f_{2}\left(\phi_{0}, \phi_{+}, \phi_{-}\right)\left(D_{\mu} \phi_{+}\right)\left(D^{\mu} \phi_{+}\right)+f_{2}\left(\phi_{0}, \phi_{-}, \phi_{+}\right)\left(D_{\mu} \phi_{-}\right)\left(D^{\mu} \phi_{-}\right) \\
& +f_{3}\left(\phi_{0}, \phi_{+}, \phi_{-}\right)\left(D_{\mu} \phi_{+}\right)\left(D^{\mu} \phi_{-}\right)^{\dagger}+f_{3}\left(\phi_{0}, \phi_{-}, \phi_{+}\right)\left(D_{\mu} \phi_{-}\right)\left(D^{\mu} \phi_{+}\right)^{\dagger} \\
& \left.+f_{4}\left(\phi_{0}, \phi_{+}, \phi_{-}\right)\left(D_{\mu} \phi_{+}\right)\left(D^{\mu} \phi_{-}\right)\right\}+ \text {H.c. },
\end{aligned}
$$

with coefficient functions $\left\{f_{i}\right\}$ depending on the fields $\phi_{0}, \phi_{ \pm}$(but not their derivatives) such that each term is $U(1)_{\mathrm{G}}$ and gauge invariant. We emphasize that the long-distance EFT (3.13) does not rely on a weak-coupling expansion. It is valid at long distances whenever the theory is in the Higgs phase. ${ }^{12}$ The $f_{1}$ terms represent wavefunction renormalizations which simply modify the overall normalizations in the energy density (3.8), and have no effect on the flux quantization condition (3.9). When evaluated on the vortex, the $f_{2}$ terms also have the same form as the long distance energy density (3.8). The $f_{3}$ and $f_{4}$ terms produce a $1 / r^{2}$ contribution to the vortex energy density proportional to

$$
\frac{v_{c}^{2}}{r^{2}}\left(n-k-\frac{\Phi}{2 \pi}\right)\left(n-\frac{\Phi}{2 \pi}\right)=\frac{v_{c}^{2}}{2 r^{2}}\left[\left(n-\frac{\Phi}{2 \pi}\right)^{2}+\left(n-k-\frac{\Phi}{2 \pi}\right)^{2}-k^{2}\right]
$$

Hence, up to holonomy-independent terms, the $f_{3}$ and $f_{4}$ terms also merely change the normalization of the tree-level energy density (3.8). Therefore, provided fluctuations are not strong enough to flip its overall sign, the holonomydependent $1 / r^{2}$ energy density has minima (with respect to $\Phi)$ at $\Phi=(2 n-k) \pi$. In particular, all minimal-circulation $(k= \pm 1)$ vortices which minimize the quantum-corrected long-distance energy density carry flux $\Phi=\pi$ modulo $2 \pi$. If fluctuations do flip the sign in front of Eq. (3.8), then the energy density becomes unbounded below as a function of $\Phi$, with no additional local minima appearing. The EFT description (3.13) therefore breaks down, signaling the departure from the Higgs phase. Therefore, within the Higgs phase, the fluctuation-induced corrections to the effective action have no effect on the flux quantization condition (3.9).

This shows that the minimal vortex expectation value $\langle\Omega(C)\rangle_{1}$ at large distance remains real and negative to all orders in perturbation theory, provided that the fluctuations are not so large that they completely destroy the Higgs phase. The size of quantum fluctuations in this model is controlled by the dimensionless parameter $e^{2} / m_{A}=$ $\mathcal{O}\left(e \lambda_{c}^{1 / 2} /\left|m_{c}\right|\right)=\mathcal{O}\left(e^{2} /\left|m_{c}\right|\right)$, where we have assumed $\lambda_{c}^{1 / 2} \sim \epsilon^{1 / 3} \sim e$ and $g_{0}, g_{c} \ll 1$ for simplicity, and hence

\footnotetext{
${ }^{12}$ More precisely, the long-distance EFT (3.13) neglects the instanton-monopole induced potential for the dual photon and, as such, is valid provided the mass $m_{A}(2.10)$ generated by the Higgs mechanism is large compared to the monopole induced photon mass $m_{\gamma}$ (2.13).
}

this conclusion about a negative value of $\langle\Omega(C)\rangle_{1}$ holds exactly whenever $m_{c}^{2} / e^{4}$ is sufficiently negative to put the theory into the Higgs phase.

As discussed earlier, quantum fluctuations do suppress the magnitude of holonomy expectation values leading to perimeter law exponential decay. By construction, this size dependence cancels in our ratio $O_{\Omega}=\langle\Omega(C)\rangle_{1} /\langle\Omega(C)\rangle$. Unbroken $\left(\mathbb{Z}_{2}\right)_{\mathrm{F}}$ symmetry [or $\left(\mathbb{Z}_{2}\right)_{\mathrm{C}}$, or reflection symmetry] in the vacuum state guarantees that the ordinary expectation value $\langle\Omega(C)\rangle$ in the denominator is real. It is easy to check that it is positive at tree level, and sufficiently small quantum fluctuations cannot make it negative. So $O_{\Omega}$ is determined by the phase of the vortex state holonomy expectation value in the numerator. The net result from this argument is that within the Higgs phase,

$$
\text { Higgs phase: } O_{\Omega}=-1 \text {, }
$$

it holds precisely. The next subsection gives useful alternative perspectives on the same conclusion.

\section{Vortex junctions, monopoles, and vortex flux quantization}

In the preceding section we analyzed the physics of vortices using effective field theory in the bulk threedimensional spacetime. This analysis showed that the minimal energy vortices carry quantized magnetic flux $\pm \pi$, and the phase of the holonomy around vortices is quantized, leading to result (3.15). We now reconsider the 
same physical questions from the perspective of an effective field theory defined on the vortex worldline. This will lead to a discussion of vortex junctions, to their interpretation as magnetic monopoles, to a connection between vortex flux quantization and Dirac charge quantization, and finally to distinct logically independent arguments for the result (3.15).

The $(0+1)$-dimensional effective field theory describing fluctuations of a vortex worldline includes two gapless modes arising from the translational moduli representing the spatial position of the vortex. The vortex effective field theory must include an additional real scalar field which may be chosen to equal the magnetic flux $\Phi$ carried by a vortex configuration. This field will serve as a coordinate along field configuration paths which interpolate between distinct vortex solutions. The field $\Phi$ appears in the 1D worldline EFT in the form

$S_{\text {vortex EFT }}=\int d t\left[c_{K}\left(\partial_{t} \Phi\right)^{2}+c_{V} V(\Phi)\right]+\cdots$.

Here $t$ is a coordinate running along the vortex worldline, $\Phi$ is dimensionless, $c_{K}$ and $c_{V}$ are low-energy constants with dimensions of inverse energy and energy, respectively, and the ellipsis represents terms with additional derivatives or couplings to other fields on the worldline.

The worldline potential $V(\Phi)$ in expression (3.16) obeys two important constraints. First, since $\left(\mathbb{Z}_{2}\right)_{\mathrm{F}}$ symmetry acts on $\Phi$ by $\Phi \rightarrow-\Phi, V(\Phi)$ is an even function. Second, Dirac charge quantization in the underlying bulk quantum field theory further constrains the possible minima of $V(\Phi)$. To see this, suppose that $V(\Phi)$ has a minimum at $\Phi=$ $\Phi_{\min } \neq 0$. Since $V(\Phi)$ is an even function, it must also have a distinct minimum at $\Phi=-\Phi_{\min }$. For generic values of the microscopic parameters, the potential $V$ is finite for all finite values of $\Phi$. This means that there exists a solution to the equation of motion for $\Phi$ in which $\Phi$ interpolates between $-\Phi_{\min }$ and $\Phi_{\min }$ as the worldline coordinate $t$ runs from $-\infty$ to $+\infty$. Suppose that this tunneling event has an action which is both UV and IR finite, so that it is meaningful to describe it within the worldline effective field theory. What is its interpretation in bulk spacetime? It has unit $U(1)_{\mathrm{G}}$ circulation at all times, but also possesses a "junction" at some finite time where the magnetic flux changes sign. For the tunneling event to have finite action, the azimuthal component of the electric field far from the vortex core must decay faster than $1 / r$. Then the flux of the field strength through a 2 -sphere surrounding the junction is simply $\Phi_{\min }-\left(-\Phi_{\min }\right)=2 \Phi_{\min }$. Comparing this to the Dirac charge quantization condition in (2.2) implies that $\Phi_{\min } \in \pi \mathbb{Z}$ when $\left(\mathbb{Z}_{2}\right)_{\mathrm{F}}$ is unbroken. ${ }^{13}$ These remarks

\footnotetext{
${ }^{13}$ If $\left(\mathbb{Z}_{2}\right)_{\mathrm{F}}$ symmetry is explicitly broken, Dirac charge quantization together with the assumption that tunneling events have finite action leads to the conclusion that any two distinct minima $\Phi_{1}, \Phi_{2}$ of $V(\Phi)$ must satisfy $\Phi_{1}-\Phi_{2} \in 2 \pi \mathbb{Z}$.
}

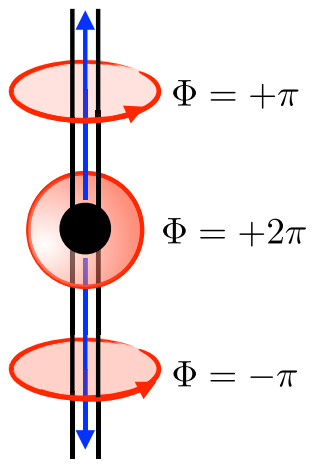

FIG. 4. A junction between the two minimal energy unitwinding vortex worldlines is a magnetic monopole with flux $2 \pi$.

imply that the worldline tunneling events can be interpreted as monopole-instantons in the $3 \mathrm{D}$ bulk, and their action must depend on the UV completion of our compact Abelian gauge theory. ${ }^{14}$

In the preceding section, we saw that in the Higgs phase minimal-energy unit-circulation vortices carry magnetic flux $\pm \pi$ at tree level. The vortex flux quantization argument in the paragraph above implies that quantum corrections cannot change this result, again leading to result (3.15). We also learn that a junction between two minimal-energy unitcirculation vortices with flux $\pi$ and $-\pi$ can be interpreted as a magnetic monopole carrying the minimal $2 \pi$ flux consistent with Dirac charge quantization, as illustrated in Fig. 4. This is the Higgs phase version of a single monopole instanton, discussed earlier, when the $\phi_{0}$ condensate has unit winding.

As noted earlier near the end of Sec. II, Higgs phase monopole-antimonopole pairs are connected by magnetic flux tubes (which can break at sufficiently large separation due to monopole-antimonopole pair creation). This is true in the absence of any vortices carrying unit $U(1)_{\mathrm{G}}$ winding. But in the presence of a unit circulation vortex, a monopole-antimonopole pair can bind to the vortex, with the monopole and antimonopole then free to separate arbitrarily along the vortex worldline. ${ }^{15}$ This is illustrated in Fig. 5. To see this, note that for fixed separation $L$ between monopole and antimonopole, the action will be lowered if the monopole and antimonopole move onto the vortex line, provided they are oriented such that adding the monopole-antimonopole flux tube to the vortex magnetic flux has the effect of merely flipping the sign of vortex

\footnotetext{
${ }^{14}$ Appendix B describes an explicit $S U(2)$ gauge theory which reduces to our $U(1)$ gauge theory at long distances, and where $S_{\mathrm{I}} \sim m_{W} / e^{2}$ with $m_{W}$ the $W$-boson mass.

${ }^{15}$ Deconfinement of magnetic monopoles on both local and semilocal vortices with and without supersymmetry has been extensively studied previously. In our model the vortices are global but the monopole deconfinement mechanism described here is essentially identical to previous discussions in, for example, Refs. [29-37].
} 


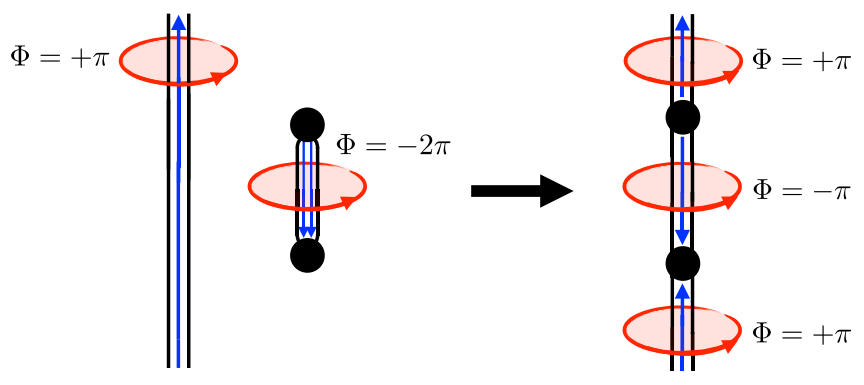

FIG. 5. Monopole-antimonopole pairs with minimal magnetic flux $2 \pi$ are confined in bulk spacetime, but such pairs are attracted to the worldline of a minimal global vortex where they become deconfined.

magnetic flux on a portion of its worldline. This eliminates the cost in action of the length $L$ flux tube initially connecting the monopole and antimonopole. As noted above, the $\left(\mathbb{Z}_{2}\right)_{\mathrm{F}}$ symmetry guarantees that the vortex action per unit length is independent of the sign of the magnetic flux. Once the monopole and antimonopole are bound to the vortex worldline, there is no longer any cost in action (neglecting exponentially falling short distance effects) to separate the monopole and antimonopole arbitrarily. In summary, the monopole-antimonopole string tension vanishes on the vortex, and magnetic monopoles are deconfined on minimal Higgs phase vortices. ${ }^{16}$

One can also regard the monopole-antimonopole pair as an instanton-anti-instanton pair in the worldline EFT (3.16). ${ }^{17}$ We now argue that this perspective leads to yet another derivation of the result (3.15). The existence of degenerate global minima with flux $\pm \Phi_{\min }$ means that the $\left(\mathbb{Z}_{2}\right)_{\mathrm{F}}$ symmetry is spontaneously broken on the worldline to all orders in perturbation theory. But nonperturbatively, the finite-action worldline instantons connecting these minima will proliferate and restore the $\left(\mathbb{Z}_{2}\right)_{\mathrm{F}}$ symmetry. As is familiar from double-well quantum mechanics, the unique minimal energy vortex state will be a symmetric linear combination of $\Phi_{\min }$ and $-\Phi_{\min }$ configurations.

From our previous arguments we know that $\Phi_{\min }=\pi$, so that both of these vortex configurations have the same -1 long distance holonomy, and none of this nonperturbative physics has any effect on the validity of the result (3.15) regarding Higgs phase vortices. But suppose that we did not already know that $\Phi_{\min }=\pi$. The existence of finiteaction tunneling events connecting the two $\Phi$ minima would imply that the minimal energy vortex state with a given winding number is unique and invariant under $\left(\mathbb{Z}_{2}\right)_{\mathrm{F}}$. Unbroken $\left(\mathbb{Z}_{2}\right)_{\mathrm{F}}$ symmetry in turn implies that the

\footnotetext{
${ }^{16}$ Provided monopoles and antimonopoles alternate along the vortex worldline. There is a direct parallel between this phenomenon and charge deconfinement in 2D Abelian gauge theories at $\theta=\pi$ (see, for example, Refs. [38-44]).

${ }^{17}$ The deconfinement of magnetic monopoles on unit-circulation vortices corresponds to the fact that the separation of an instanton-anti-instanton pair is a quasizero mode.
}

holonomy expectation value in the minimal vortex state is purely real. Therefore, on symmetry grounds alone, our observable $O_{\Omega}$ is quantized to be either +1 or -1 . Our analysis in the weakly coupled regime serves to establish that in the Higgs phase the value is -1 , and we again arrive at result (3.15).

\section{C. $O_{\Omega}$ in the $U(1)_{\mathrm{G}}$-broken confining regime}

We now turn to a consideration of holonomies around vortices in the $U(1)_{\mathrm{G}}$-broken confining phase. Once again, it is useful to consider the appropriate effective field theory deep in this regime, near the SE corner of the phase diagram of Fig. 1.

Suppose that $m_{c}^{2} \gg e^{4}$. Given the scale separation, it is useful to integrate out the charged fields. The resulting effective action retains the gauge field and neutral scalar $\phi_{0}$ and has the form

$$
\begin{aligned}
S_{\mathrm{eff}}= & \int d^{3} x\left[\frac{1}{4 e^{2}} F_{\mu \nu}^{2}+V_{\mathrm{m}}(\sigma)+\left|\partial_{\mu} \phi_{0}\right|^{2}+V\left(\left|\phi_{0}\right|\right)\right. \\
& \left.+\frac{a}{m_{c}^{2}}\left|\phi_{0}\right|^{2} F_{\mu \nu}^{2}+\cdots\right]
\end{aligned}
$$

where the ellipsis denotes higher dimension terms involving additional powers of fields and derivatives. The dimension five term shown explicitly, with coefficient $a$, is the lowest dimension operator coupling the gauge and neutral scalar fields. This term describes "Raleigh scattering" processes in which photons scatter off fluctuations in the magnitude of $\phi_{0}$. Within this EFT, the $\left(\mathbb{Z}_{2}\right)_{\mathrm{F}}$ symmetry simply flips the sign of the gauge field and hence forbids all terms involving odd powers of the gauge field strength.

When $m_{0}^{2}$ is sufficiently negative so that the $U(1)_{\mathrm{G}}$ symmetry is spontaneously broken and $\phi_{0}$ condenses, the leading effect of the $\left|\phi_{0}\right|^{2} F^{2}$ coupling is merely to shift the value of the gauge coupling by an amount depending on the condensate $v_{0} \equiv\left\langle\phi_{0}\right\rangle$,

$$
\frac{1}{e^{2}} \rightarrow \frac{1}{e^{\prime 2}} \equiv \frac{1}{e^{2}}+\frac{4 a\left|v_{0}\right|^{2}}{m_{c}^{2}} .
$$

This is a small shift of relative size $\mathcal{O}\left(e^{4} / m_{c}^{2}\right)$ within the domain of validity of this effective description. The $\left(\mathbb{Z}_{2}\right)_{\mathrm{F}}$ symmetry (or parity) guarantees that the neutral scalar condensate cannot source the gauge field strength, so the magnetic field $B \equiv \frac{1}{2} \epsilon_{i j} F^{i j}(i, j=1,2)$ must have a vanishing expectation value.

Within this $U(1)_{\mathrm{G}}$ broken phase, there are vortex configurations in which the condensate $\left\langle\phi_{0}\right\rangle$ has a phase which winds around the vortex, while its magnitude decreases in the vortex core, vanishing at the vortex center. As far as the gauge field is concerned, one sees from the effective action (3.17) that the only effect this has is to modulate the gauge coupling, effectively undoing the shift (3.18) in the vortex core. But such coupling 
renormalizations, or dielectric effects, do not change the fact that the effective action is an even function of magnetic field which is minimized at $B=0$. In other words, even in the presence of vortices, the neutral scalar field does not source a magnetic field. And consequently, both the vacuum state and minimal energy vortex states are invariant under the $\left(\mathbb{Z}_{2}\right)_{\mathrm{F}}$ symmetry.

Once again, invariance of both the vacuum and the vortex states under the $\left(\mathbb{Z}_{2}\right)_{\mathrm{F}}$ symmetry implies that holonomy expectation values in both states are real, and hence our observable $O_{\Omega}$ must be either +1 or -1 . The Abelian gauge field holonomy is, of course, nothing but the exponential of the magnetic flux, $\Omega(C)=e^{i \oint_{C} A}=$ $e^{i \int_{S} B}=e^{i \Phi_{B}}$ (with contour $C$ the boundary of disk $S$ ). The above EFT discussion shows that deep in the confining $U(1)_{\mathrm{G}}$-broken phase the influence of a vortex on the magnetic field is tiny, and hence $\langle\Omega(C)\rangle_{1}$ is positive, implying that $O_{\Omega}=+1$. And once again, by analyticity, this result must hold throughout the confining $U(1)_{\mathrm{G}^{-}}$ broken phase. In summary,

$$
U(1)_{\mathrm{G}} \text {-broken confining phase: } O_{\Omega}=+1
$$

is an exact result within this phase.

\section{Higgs-confinement phase transition}

We have seen that $O_{\Omega}$ has constant magnitude but changes sign between the Higgs and confining, $U(1)_{\mathrm{G}^{-}}$ broken regimes; it cannot be a real-analytic function of $m_{c}^{2}$. Hence, there must be at least one phase transition as a function of $m_{c}^{2}$. A single phase transition would be associated with an abrupt jump of $O_{\Omega}$ from -1 to 1 at some critical value of $m_{c}^{2}$. If instead $O_{\Omega}$ equals -1 for charged mass squared below some value $m_{c}^{2}<\left(m_{c}^{2}\right)_{A}$, equals +1 above a different value $\left(m_{c}^{2}\right)_{B}<m_{c}^{2}$, and continuously interpolates from -1 to +1 in the intervening interval $\left(m_{c}^{2}\right)_{A}<m_{c}^{2}<\left(m_{c}^{2}\right)_{B}$, this would indicate the presence of two phase transitions bounding an intermediate phase in which the $\left(\mathbb{Z}_{2}\right)_{\mathrm{F}}$ symmetry is spontaneously broken. [This follows since, as discussed above, unbroken $\left(\mathbb{Z}_{2}\right)_{\mathrm{F}}$ symmetry implies that $O_{\Omega}$ must equal \pm 1 .]

In much of parameter space, phase transitions in our model occur at strong coupling and are not amenable to analytic treatment. But the theory becomes weakly coupled when the masses $\left|m_{c}^{2}\right|$ and $\left|m_{0}\right|^{2}$ are sufficiently large. Specifically, we will assume that the dimensionful couplings $\left|\lambda_{c}\right|,\left|\lambda_{0}\right|$, and $e^{2}$ are all small relative to the masses $\left|m_{c}\right|$ and $\left|m_{0}\right|$, the cubic coupling obeys $\epsilon \ll \min \left(\left|m_{c}\right|^{3 / 2},\left|m_{0}\right|^{3 / 2}\right)$, and the sextic couplings are small, $g_{c}, g_{0} \ll 1$. If a first order transition lies within this region, then simple analytic arguments suffice to identify and locate the transition.

A first order transition involving a complex scalar $\phi$ with $U(1)$ symmetry requires multiple local minima in the effective potential viewed as a function of $|\phi|$. In four dimensions, a renormalizable scalar potential is quartic and, as a function of $|\phi|$, has at most a single local minimum. So to find a first order phase transition in a weakly coupled four-dimensional $U(1)$ invariant scalar theory one must either be abnormally sensitive to higher order nonrenormalizable terms (and thus probing cutoff-scale physics) or else be reliant on a one-loop or higher order calculation producing nonanalytic terms such as $|\phi|^{2} \log |\phi|$. This is illustrated by the classic Coleman and Weinberg analysis [45]. But in three spacetime dimensions, renormalizable scalar potentials are sextic, and $U(1)$ invariant sextic potentials can easily have multiple local minima. Consequently, a tree-level analysis can suffice to demonstrate the existence of a first order phase transition, in a renormalizable theory, without any need to consider higher order corrections.

Let us see how this works in our model. Consider the region where $m_{0}^{2}, \lambda_{c}$, and $\lambda_{0}$ are all negative. For simplicity, let us also suppose that $e^{2} \ll\left|\lambda_{c}\right|,\left|\lambda_{0}\right|$, and $\epsilon \ll e^{3} \ll$ $\min \left(\left|m_{c}\right|^{3 / 2},\left|m_{0}\right|^{3 / 2}\right)$. In Fig. 6 we show contour plots of the scalar potential as a function of $\phi_{c} \equiv \phi_{+}$and $\phi_{0}$, with $\phi_{-}=\left|\phi_{+}\right|$, as $m_{c}^{2} / e^{4}$ is varied. The figure shows that the potential has multiple local minima with relative ordering that changes as $m_{e}^{2} / e^{4}$ is varied with all other parameters held fixed. With the parameter choices given in the caption of Fig. 6, the figure shows the existence of a strong first order phase transition between $U(1)_{\mathrm{G}}$-broken confining and $U(1)_{\mathrm{G}}$-broken Higgs states in the regime where $m_{0}^{2} / e^{4}$ is large and negative and $m_{c}^{2} / e^{4}$ is large and positive. Correspondingly, the change in the derivative of the energy density with respect to the charged scalar mass squared in units of $e^{2}, e^{-2} \Delta\left(\partial \mathcal{E} / \partial m_{c}^{2}\right)$, is large across the transition. For the parameter values used in Fig. 6 one finds $e^{-2} \Delta\left(\partial \mathcal{E} / \partial m_{c}^{2}\right)=2 \Delta \phi_{c}^{2} / e^{2} \approx 127 \gg 1$. This behavior is generic. The effective masses (i.e., curvatures of the potential) at the minima are comparable to the input mass parameters, so there are no near-critical fluctuations and the phase transition is reliably established at weak coupling.

Finally, the analysis of the previous subsections shows that our vortex holonomy order parameter $O_{\Omega}$ changes sign across this phase transition, confirming that the abrupt change in this "topological" order parameter is associated with a genuine thermodynamic phase transition.

As one moves into the interior of the $\left(m_{0}^{2}, m_{c}^{2}\right)$ phase diagram, out of the weakly coupled periphery, we certainly expect this direct correlation between a jump in our vortex order parameter and a thermodynamic phase transition to persist. But one may contemplate whether this association could cease to apply at some point in the interior of the $U(1)_{\mathrm{G}}$ spontaneously broken domain. In general, a line of first order phase transitions which is not associated with any change in symmetry realization can have a critical end point (as seen in the phase diagram of water). Could our model have such a critical end point, beyond which the first order transition becomes a smooth crossover as probed by 

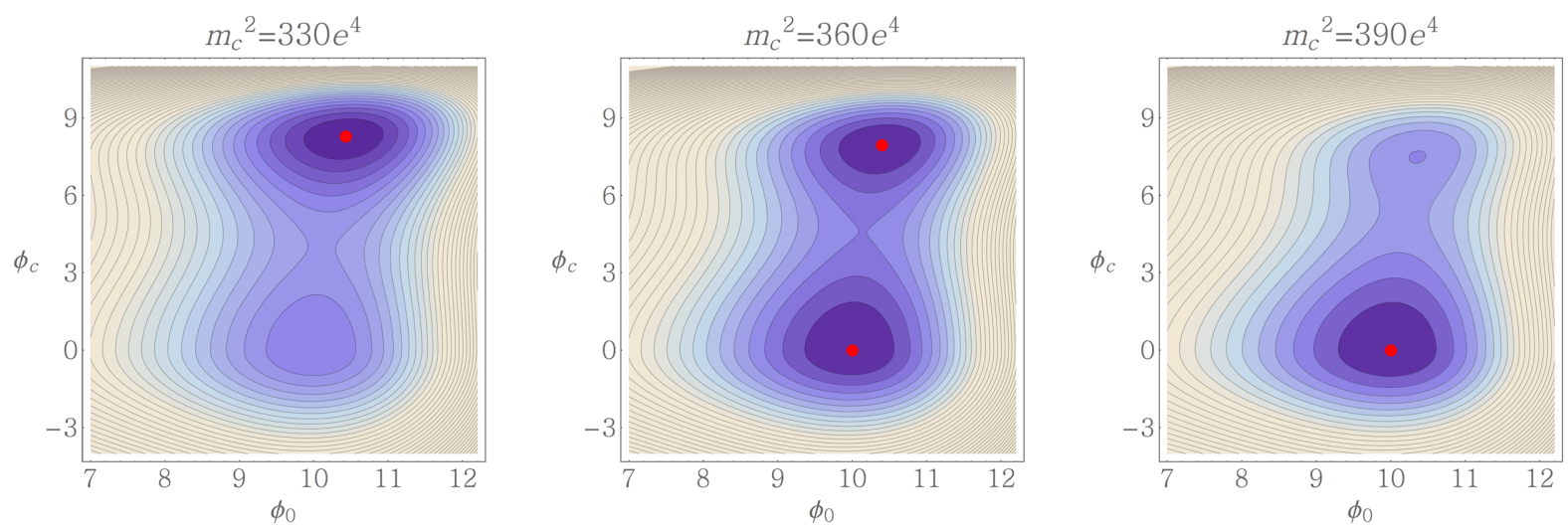

FIG. 6. Contour plots of the tree-level scalar effective potential at three different values of $m_{c}^{2}$ in the vicinity of the first order Higgsconfinement phase transition. We have used gauge and global symmetries to choose the phases of the scalar fields such that the potential can be interpreted as a function of $\phi_{c} \equiv \phi_{+}$and $\phi_{0}$, with $\phi_{-}=\left|\phi_{+}\right|$. We have set $m_{0}^{2}=-200 e^{4}, \epsilon=40 e^{3}, \lambda_{c}=\lambda_{0}=-5 e^{2}$, and $g_{c}=g_{0}=0.04$. Decreasing values of the scalar potential are colored with darker colors, and global minima are marked with red dots. Note that the global minimum is degenerate when $m_{c}^{2} \approx 360 e^{4}$, and the location of the global minimum jumps as $m_{c}^{2} \mathrm{crosses}$ this value, from a point where the charged fields are condensed to one where they are not condensed. This shows the presence of a strong first order Higgs-confinement phase transition, with the $U(1)_{\mathrm{G}}$ global symmetry spontaneously broken on both sides of the transition.

any local observable? If so, there would necessarily remain some continuation of the phase transition line across which our topological observable $O_{\Omega}$ continues to flip sign, but all local observables remain smooth. What would be necessary for such a scenario to take place?

First, note that the magnetic flux carried by vortices can change in steps of $2 \pi$ due to alternating monopole-instanton fluctuations appearing along the vortex worldline, but such processes do not affect the sign of the holonomy around a vortex. At the transition between the Higgs and confining phases the magnetic flux carried by minimalwinding vortices changes by $\pi$ (modulo $2 \pi$ ). It is very tempting to expect such a sudden change in the vortex magnetic flux to imply nonanalyticity in the IR-finite core energy of a vortex, or equivalently the vortex fugacity. Whenever the $U(1)_{\mathrm{G}}$ symmetry is spontaneously broken, the equilibrium state of the system will contain a nonzero density of vortices and antivortices due to quantum fluctuations. If the minimal vortex energy is nonanalytic, this will in turn induce nonanalyticity in the true ground state energy density. [This argument ceases to apply only when the vortex density reaches the point where vortices condense, thereby restoring the $U(1)_{\mathrm{G}}$ symmetry.] In other words, if nonanalyticities in vortex magnetic flux imply nonanalyticity in the vortex energy, our vortex holonomy observable functions as a useful order parameter, identifying thermodynamically distinct gapless phases.

There is a possible loophole in the above argument: what if the change in vortex magnetic flux is caused by a level crossing between vortices of flux $\pi$ and $0(\bmod 2 \pi) ?^{18}$ Such a level crossing could produce nonanalyticy in our vortex

\footnotetext{
${ }^{18}$ We are grateful to N. Seiberg for useful discussions on this issue.
}

holonomy observable without being associated with nonanalyticity in the ground state energy or other thermodynamic observables. However, for such a level crossing to be possible, a (metastable) unit-winding vortex with flux 0 $(\bmod 2 \pi)$ would need to exist in the Higgs phase and become degenerate with the flux $\pi(\bmod 2 \pi)$ unit-winding vortex as one varies parameters. Our analysis of the quantum effective action for the vortex holonomy shows that, within the domain of validity of the effective action (3.13), there simply are no static solutions describing unitwinding vortices with flux equal to $0 \bmod 2 \pi$ in the Higgs phase. The quantum effective action (3.13) is a valid long distance description throughout the Higgs regime relying, essentially, only on a large ratio of the distance scale of interest to microscopic scales. However, Eq. (3.13) does not take into account monopole-instanton effects, so it necessarily ceases to be applicable in a transition region between the confining and Higgs regimes where the Higgs mass scale $m_{A} \sim e^{2} v_{c}^{2}$ becomes comparable to the monopoleinduced photon mass scale $m_{\gamma}^{2} \sim\left(\mu_{\mathrm{UV}}^{3} / e^{2}\right) e^{-S_{\mathrm{I}}}$. This region in parameter space can be made arbitrarily small by increasing $S_{\mathrm{I}}$. For the level-crossing scenario to take place, one would need to envision that as we go from the Higgs regime toward the confining regime, a flux $2 \pi$ minimalwinding vortex has to appear with a higher energy than a $\pi$ flux minimal-winding vortex, and then cross it in energy, all within this arbitrarily small region. Moreover, this phenomenon would have to take place only in the strongly coupled region of parameter space, because it certainly does not happen in the weakly coupled domain, illustrated in Fig. 6, where we have shown the existence of a first order phase transition. So while we cannot absolutely rule out this level-crossing scenario, in our view it requires enough conspiracies to seem very far-fetched. 
This concludes our arguments for the presence of at least one phase transition curve separating the SE and $\mathrm{W}$ regions of Fig. 1.

\section{E. Explicit breaking of flavor permutation symmetry}

We now generalize our model to include operators which break the $\left(\mathbb{Z}_{2}\right)_{\mathrm{F}}$ symmetry explicitly. The simplest such term is just a mass perturbation giving the two charged fields $\phi_{+}$and $\phi_{-}$distinct masses $m_{+}$and $m_{-}$. Let

$$
m_{\mathrm{avg}}^{2} \equiv \frac{1}{2}\left(m_{+}^{2}+m_{-}^{2}\right), \quad \Delta \equiv \frac{1}{e^{4}}\left(m_{+}^{2}-m_{-}^{2}\right)
$$

denote the average mass squared and a measure of their difference, respectively. We will examine the dependence of physics on $m_{\mathrm{avg}}^{2} / e^{4}$ with $\Delta>0$ held fixed.

If $\Delta$ is sufficiently large, then there are two seemingly different regimes where no global symmetries are spontaneously broken: one where no scalar fields are condensed, and another where only $\phi_{-}$is condensed. The latter regime is not a distinct phase as condensation of the charged field $\phi_{-}$, by itself, does not imply a nonvanishing expectation value of any physical order parameter. In fact, these two regimes are smoothly connected to each other and are trivial in the sense that they have a mass gap and a vacuum state which is invariant under all global symmetries.

The more interesting regimes of the model are those with spontaneously broken $U(1)_{\mathrm{G}}$ symmetry. The cubic term in the action $\epsilon \phi_{0} \phi_{+} \phi_{-}+$H.c. ensures that there is no regime where $\phi_{0}$ and only one of the two charged fields are condensed. Hence we only need to consider two regimes with spontaneously broken $U(1)_{\mathrm{G}}$ symmetry: one where all scalar fields are condensed, and another where only the neutral scalar $\phi_{0}$ is condensed.

\section{Higgs regime}

Consider the Higgs regime where $-m_{\mathrm{avg}}^{2} \gg e^{4}$ and all scalars are condensed. The tree-level long-distance energy density that determines the holonomy around a $U(1)_{\mathrm{G}}$ vortex of winding number $k$ is given by an obvious generalization of Eq. (3.8),

$$
\begin{aligned}
\mathcal{E}(r)= & \frac{v_{+}^{2}}{r^{2}}\left(n-k-\frac{\Phi}{2 \pi}\right)^{2}+\frac{v_{-}^{2}}{r^{2}}\left(-n+\frac{\Phi}{2 \pi}\right)^{2}+\frac{v_{0}^{2} k^{2}}{r^{2}} \\
& +\mathcal{O}\left(r^{-4}\right) .
\end{aligned}
$$

Due to the explicit breaking of $\left(\mathbb{Z}_{2}\right)_{\mathrm{F}}$, the magnitudes of the charged scalar expectation values $v_{+}$and $v_{-}$are no longer equal; let us denote their average by $v_{\text {avg. }}$. For given values of $k$ and $n$, minimizing the above energy density yields

$$
\Phi=\left(2 n-k \frac{v_{+}^{2}}{v_{\text {avg }}^{2}}\right) \pi,
$$

and $\mathcal{E}=\left(\frac{1}{2} \frac{v_{+}^{2} v_{-}^{2}}{v_{\text {avg }}^{2}}+v_{0}^{2}\right) k^{2} / r^{2}+\mathcal{O}\left(r^{-4}\right)$. Due to the explicit breaking of $\left(\mathbb{Z}_{2}\right)_{\mathrm{F}}$, there are no longer two degenerate minimal-winding vortices at tree level. Suppose $v_{-}^{2}<v_{+}^{2}$ without loss of generality. Then the unique minimal energy unit-winding vortex (corresponding to $k=1, n=1$ ) carries magnetic flux

$$
\Phi=\frac{v_{-}^{2}}{v_{\mathrm{avg}}^{2}} \pi
$$

which is no longer quantized in units of $\pi$. This means that the holonomy encircling a vortex, $\langle\Omega(C)\rangle_{1}=e^{i \Phi}$, is no longer real.

The ordinary holonomy expectation value in the denominator of $O_{\Omega}$ necessarily remains real and positive due to the continuing presence of unbroken $\left(\mathbb{Z}_{2}\right)_{\mathrm{C}}$ symmetry. Consequently, in this tree-level analysis, our nonlocal order parameter $O_{\Omega}$ is a nontrivial phase which differs from both -1 and +1 . Small quantum corrections cannot bring the vortex magnetic flux (3.23) to 0 , so this conclusion must hold generically throughout the phase which extends inward from the weakly coupled regime. In particular,

Higgs phase without $\left(\mathbb{Z}_{2}\right)_{\mathrm{F}}$ symmetry: $O_{\Omega} \neq 1$.

Following the analysis in the next subsection, we will see that one can actually interpret the condition (3.24) as a gauge-invariant criterion defining the Higgs phase.

\section{2. $U(1)_{\mathrm{G}}$-broken confining regime}

Now consider the regime where neither charged scalar field is condensed. When $m_{\text {avg }}^{2}$ is large (compared to other scales) one may integrate out both charged fields and the effective description of the theory is given by Eq. (3.17), with $m_{c}$ now defined as the mass of the lightest charged field, $m_{c}=\min \left(m_{+}, m_{-}\right)$, plus additional higher dimension operators which are no longer forbidden by the $\left(\mathbb{Z}_{2}\right)_{\mathrm{F}}$ symmetry. Writing out the lowest dimension such term explicitly, we have

$$
\begin{aligned}
S_{\mathrm{eff}}= & \int d^{3} x\left[\frac{1}{4 e^{2}} F_{\mu \nu}^{2}+V_{\mathrm{m}}(\sigma)+\left|\partial_{\mu} \phi_{0}\right|^{2}+V\left(\left|\phi_{0}\right|\right)\right. \\
& \left.+\frac{a}{m_{c}^{2}}\left|\phi_{0}\right|^{2} F_{\mu \nu}^{2}+\frac{b}{m_{c}^{2}} S_{\mu \nu} F^{\mu \nu}+\cdots\right]
\end{aligned}
$$

where the "polarization" $S_{\mu \nu} \equiv \frac{i}{2}\left[\left(\partial_{\mu} \phi_{0}^{\dagger}\right)\left(\partial_{\nu} \phi_{0}\right)-\right.$ $\left.\left(\partial_{\nu} \phi_{0}^{\dagger}\right)\left(\partial_{\mu} \phi_{0}\right)\right]$. To examine the effect of this new dimension-five $\left(\mathbb{Z}_{2}\right)_{\mathrm{F}}$-odd term $S \cdot F$ in the presence of vortices, it will prove helpful to integrate by parts and rewrite it as direct coupling between the gauge field and a current, $\int d^{3} x A_{\mu} J_{\text {eff }}^{\mu}$, with the current built out of gradients of the neutral scalar $\phi_{0}$, 


$$
J_{\text {eff }}^{\mu}=\frac{2 b}{m_{c}^{2}} \partial_{\nu} S^{\mu \nu}
$$

This current is automatically conserved, $\partial_{\mu} J_{\text {eff }}^{\mu}=0$, as required by gauge invariance. ${ }^{19}$ Now consider the minimal vortex configuration where the neutral scalar has a spatially varying magnitude and phase, $\phi_{0}=v_{0} f_{0}(r) e^{i \theta}$. This induces a nonzero antisymmetric $S_{\mu \nu}$ with

$$
S_{r \theta}=v_{0}^{2} \frac{f_{0}(r) f_{0}^{\prime}(r)}{r} .
$$

This polarization is localized on the vortex core [with an $\mathcal{O}\left(r^{-4}\right)$ power-law tail]. The associated current $J_{\text {eff }}^{\mu}$ has an azimuthal component, $J_{\text {eff }}^{\theta}(r)=\frac{2 b v_{0}^{2}}{m_{c}^{2}} \partial_{r}\left[f_{0}(r) f_{0}^{\prime}(r) / r\right]$. As in any solenoid, this current sources a magnetic field which is also localized within the vortex core, i.e., $r \lesssim\left|m_{0}\right|^{-1}$, up to an $\mathcal{O}\left(r^{-4}\right)$ tail.

What does all of this mean for holonomies around vortices? There are several distinct physical length scales in the $U(1)_{\mathrm{G}}$-broken confining phase. Recall that the nonperturbative monopole-instanton induced contribution to the action depends on the classical action $S_{\text {I }}$ of a monopole-instanton and the scale $\mu_{\mathrm{UV}}$ which is set by the inverse length scale of the monopole core, $S_{\text {monopole }}=$ $\int d^{3} x V_{\mathrm{m}}(\sigma)=-\int d^{3} x \mu_{\mathrm{UV}}^{3} e^{-S_{\mathrm{I}}} \cos (\sigma)$. This term is responsible for linear confinement with a string tension $T \sim e^{2} m_{\gamma} \sim \sqrt{e^{2} \mu_{\mathrm{UV}}^{3}} e^{-S_{\mathrm{I}} / 2}$. Suppose that $T^{1 / 2} \ll m_{c}$, as is the case in the weakly coupled portion of the phase. The possibility of charged scalar pair production implies that sufficiently long strings can break. The string-breaking length,

$$
L_{\mathrm{br}} \equiv 2 m_{c} / T
$$

characterizes the length scale beyond which string-breaking effects cannot be neglected. Hence, linear confinement and area law behavior for Wilson loops only hold for intermediate distance scales between $T^{-1 / 2}$ and $L_{\mathrm{br}}$.

For our purposes, the quantity of primary interest is the holonomy for a circular contour around a vortex when the contour radius $r$ exceeds the largest intrinsic scale of the theory, $r \gg L_{\mathrm{br}}$. However, let us work up to this case by considering holonomies calculated on circles of progressively increasing size.

\footnotetext{
${ }^{19}$ Alternatively, one might be tempted to eliminate this term, which induces mixing between $S_{\mu \nu}$ and $F_{\mu \nu}$, by making a suitable redefinition of the gauge field. But for our purposes such a field redefinition is unhelpful as it complicates the evaluation of holonomies, effectively introducing a current-current interaction between the $U(1)_{\mathrm{G}}$ current and the current associated with a heavy electrically charged probe particle used to measure the holonomy.
}

Consider a circular contour $C$ with a unit-winding $\phi_{0}$ vortex at its center. To begin, suppose that the radius $r$ of the contour $C$ is large compared to the coherence length $\xi \sim 1 /\left|m_{0}\right|$ but small compared to $T^{-1 / 2}$, the inverse dual photon mass. Then confinement and monopole effects can be ignored, and a calculation of the magnetic flux using Eqs. (3.25)-(3.27) gives

$$
\langle\Omega(C)\rangle_{1}=e^{-2 \pi r \mu^{\prime}} e^{i \Phi}, \quad \xi \ll r \ll T^{-1 / 2},
$$

where $\mu^{\prime}$ is a scheme dependent renormalization scale and the flux is given by

$$
\Phi=2 \pi b\left(\frac{e v_{0}}{m_{c}}\right)^{2} .
$$

So, for contours encircling vortices in this "inner" distance regime (but still far outside the vortex core), we find that

$$
\frac{\langle\Omega(C)\rangle_{1}}{\langle\Omega(C)\rangle}=e^{i \Phi}, \quad \xi \ll r \ll T^{-1 / 2}
$$

Next, suppose that the contour radius satisfies $T^{-1 / 2} \ll r \ll L_{\mathrm{br}}$. The dual photon mass term is important in this regime. To compute the behavior of the Wilson loop, we recall the usual prescription of Abelian duality (see, e.g., Refs. $[25,46])$ : an electric Wilson loop along a contour $C$ maps to a configuration of the dual photon with a $2 \pi$ monodromy on curves that link $C$. A very large Wilson loop in the $x-y$ plane can be described by a configuration of $\sigma$ which, well inside the loop, is purely $t$ dependent, with $\sigma$ vanishing as $t \rightarrow \pm \infty$ while having a $2 \pi$ discontinuity at $t=0$. In the Abelian dual description, the effective action (3.25) then takes the form

$$
\begin{aligned}
S_{\mathrm{eff}}= & \int d^{3} x\left[\left|\partial \phi_{0}\right|^{2}+V\left(\left|\phi_{0}\right|\right)\right. \\
& +\frac{e^{2}}{8 \pi^{2}}(\partial \sigma)^{2}\left(1-\frac{4 a e^{2}}{m_{c}^{2}}\left|\phi_{0}\right|^{2}\right)-\mu_{\mathrm{UV}}^{3} e^{-S_{\mathrm{I}}} \cos (\sigma) \\
& \left.+\frac{i b e^{2}}{2 \pi m_{c}^{2}} \epsilon^{\mu \nu \rho} S_{\mu \nu} \partial_{\rho} \sigma+\cdots\right] .
\end{aligned}
$$

On the vortex configuration, the final $b$ term becomes

$$
\frac{i b e^{2}}{2 \pi m_{c}^{2}} \epsilon^{\mu \nu \alpha} S_{\mu \nu} \partial_{\alpha} \sigma=\frac{i b}{\pi}\left(\frac{e v_{0}}{m_{c}}\right)^{2} \frac{f_{0}(r) f_{0}^{\prime}(r)}{r} \partial_{t} \sigma .
$$

Since the vortex configuration is time independent, the integral of this term only receives a contribution from the $2 \pi$ discontinuity in $\sigma$ at $t=0$. Evaluating the effective action (3.32) on this solution gives a result for the holonomy expectation value of

$$
\langle\Omega(C)\rangle_{1}=e^{-2 \pi r \mu^{\prime}} e^{-T \pi r^{2}} e^{i \Phi}, \quad T^{-1 / 2} \ll r \ll L_{\mathrm{br}},
$$


showing an area-law decrease in magnitude together with the same phase (3.30) appearing in smaller holonomy loops. Of course, without a vortex the $b$ term vanishes and the holonomy expectation shows a pure area-law decrease with no phase,

$$
\langle\Omega(C)\rangle=e^{-2 \pi r \mu^{\prime}} e^{-T \pi r^{2}}, \quad T^{-1 / 2} \ll r \ll L_{\mathrm{br}} .
$$

Consequently, for this "intermediate" range of circle sizes we again find

$$
\frac{\langle\Omega(C)\rangle_{1}}{\langle\Omega(C)\rangle}=e^{i \Phi}, \quad T^{-1 / 2} \ll r \ll L_{\mathrm{br}} .
$$

Now we are finally ready to consider the most interesting regime of holonomy contours, those with $r \gg L_{\mathrm{br}}$. First, consider the unconstrained vacuum expectation value. Due to the presence of heavy dynamical charged excitations, Wilson loop expectation values contain a sum of area-law and perimeter-law contributions, but the perimeter-law contribution dominates in the long-distance regime,

$$
\begin{aligned}
\langle\Omega(C)\rangle & =e^{-2 \pi r \mu^{\prime}}\left(e^{-T \pi r^{2}}+e^{-2 \pi r m_{c}}\right) \sim e^{-2 \pi r\left(m_{c}+\mu^{\prime}\right)}, \\
L_{\mathrm{br}} & \ll r .
\end{aligned}
$$

(Here, irrelevant prefactors are neglected.) Physically, this Wilson loop expectation describes a process where a unit test charge and anticharge are inserted at some point, separated, and then recombined after following semicircular worldlines (in Euclidean space) forming two halves of the contour $C$. The second perimeter-law term arises from contributions in which dynamical charges of mass $m_{c}$ are pair created and dress the test charge and anticharge to create two bound gauge-neutral "mesons." These mesons have a physical size of order $\ell_{\text {meson }} \sim$ $\min \left(T^{-1 / 2},\left(e^{2} m_{c}\right)^{-1 / 2}\right)$, and experience no long range interactions. ${ }^{20}$ Once the loop size exceeds $L_{\mathrm{br}}$, pair creation of dynamical charges of mass $m_{c}$ and the associated meson formation becomes the dominant process.

Finally, suppose that this very large contour $C$ encircles a minimal vortex. Then the area-law contribution to the holonomy expectation acquires the phase $\Phi$, in exactly the same manner described above. In contrast, the perimeterlaw contribution arises from fluctuations of the charged fields within distances of order of $\ell_{\text {meson }}$ from any point on the contour $C$. The amplitude for such screening fluctuations, and consequent meson formation, must be completely insensitive to the presence of a vortex very far away at the center of the loop. Consequently, in the presence of a vortex the two different contributions to the holonomy expectation value have different phases,

$$
\langle\Omega(C)\rangle_{1}=e^{-2 \pi r \mu^{\prime}}\left(e^{-T \pi r^{2}} e^{i \Phi}+e^{-2 \pi r m_{c}}\right) .
$$

Once again, in the long distance regime, $r \gg L_{\mathrm{br}}$ the stringbreaking or perimeter-law term dominates.

Combining the vortex holonomy expectation (3.38) with the vacuum expectation (3.37), we find that their ratio, in the long distance regime, equals 1 up to exponentially small corrections,

$$
\frac{\langle\Omega(C)\rangle_{1}}{\langle\Omega(C)\rangle}=1+\mathcal{O}\left(e^{-T \pi r^{2}\left(1-L_{\mathrm{br}} / r\right)}\left(e^{i \Phi}-1\right)\right)
$$

Hence, the large $r$ limit defining our vortex observable $O_{\Omega}$ exists and yields the simple result:

$$
U(1)_{\mathrm{G}} \text {-broken confining phase without }\left(\mathbb{Z}_{2}\right)_{\mathrm{F}} \text { symmetry: } O_{\Omega}=+1 \text {. }
$$

Being strictly constant (i.e., with no dependence whatsoever on microscopic parameters), this result must hold exactly throughout the phase connected to the weakly coupled confining $U(1)_{\mathrm{G}}$-broken regime. Any deviation from $O_{\Omega}=+1$ must signal a phase transition.

\section{Summary}

Let us take stock of what we have learned about the relation between the Higgs and confining $U(1)_{\mathrm{G}}$-broken regimes in the absence of the $\left(\mathbb{Z}_{2}\right)_{\mathrm{F}}$ symmetry. So long as the $U(1)_{\mathrm{G}}$ global symmetry is spontaneously broken, there is no way to distinguish the Higgs and confining regimes

\footnotetext{
${ }^{20}$ When $m_{c} \gg T^{1 / 2} / e^{2}$, the dressed test charges are analogous to charmed $B$ mesons in QCD and can be described as $2+1 \mathrm{D}$ Coulomb bound states (see, e.g., Ref. [47]).
}

within the Landau paradigm using local order parameters. But our vortex holonomy order parameter does distinguish them. Consider the theory with large positive $m_{\text {avg }}^{2}$, in its regime where $U(1)_{\mathrm{G}}$ is spontaneously broken due to the dynamics of the neutral scalar sector, and imagine progressively decreasing $m_{\mathrm{avg}}^{2} / e^{4}$. Initially, for large positive $m_{\text {avg }}^{2} / e^{4}$, the gauge field holonomy calculated on arbitrarily large circles around $U(1)_{\mathrm{G}}$ vortices is trivial, dominated by perimeter-law contributions, and our order parameter $O_{\Omega}=+1$. But once $m_{\mathrm{avg}}^{2} / e^{4}$ decreases sufficiently, the charged scalars condense. Then the holonomy around vortices acquires a nontrivial phase, with $O_{\Omega}$ first deviating from 1 at some critical value of $m_{\mathrm{avg}}^{2} / e^{4}$. The same reasoning as in Sec. IIID implies that this nonanalytic behavior in $O_{\Omega}$ should also signal a genuine phase transition. 


\section{QCD AND THE HYPOTHESIS OF QUARK-HADRON CONTINUITY}

A central topic in strong interaction physics is understanding the phase structure of QCD as a function of baryon number density, or equivalently as a function of the chemical potential $\mu_{B}$ associated with the $U(1)_{B}$ baryon number symmetry. (For reviews see, for example, Refs. $[26,48]$.) At low (nuclear) densities, or small $\mu_{B}$, it is natural to describe the physics in terms of nucleons, while at large $\mu_{B}$ a description in terms of quark matter is appropriate thanks to asymptotic freedom. Are "confined" nuclear matter and "deconfined" quark matter sharply distinct phases of matter, necessarily separated by at least one phase transition, or might they be smoothly connected, similar to the gas and liquid phases of water?

Following Schäfer and Wilczek [24], we focus on the behavior of QCD with three flavors of quarks having a common mass $m_{q}$, so that there is a vectorlike $S U(3)$ flavor symmetry. We ignore the weak, electromagnetic, and gravitational interactions. Some readers may wonder why it is especially interesting to consider the limit of QCD with $S U$ (3) flavor symmetry. Physically there are, of course, six quark flavors in the Standard Model. But the three heaviest quark flavors (charm, bottom, and top) are so heavy that it is an excellent approximation to ignore them entirely when considering the possible continuity between nuclear matter and quark matter. The three lightest quark flavors (up, down, and strange) have distinct masses in nature, so there is no exact global $S U(3)$ symmetry acting on the light quark fields. However, in practice the strength of $S U(3)$ flavor symmetry breaking is not terribly large, since none of the three lightest quarks are heavy compared to the strong scale $\Lambda_{\mathrm{QCD}}$. So one motivation to study the $S U(3)$ flavor symmetric limit of QCD is that the physics is simplest in this limit, and at the same time it is a useful starting point for much phenomenology.

There is also a more theoretical justification for focusing on the $S U(3)$ flavor symmetric limit. Suppose that the up and down quarks are approximately degenerate in mass, but $S U$ (3) flavor symmetry is broken because the strange quark is heavier, as is the case in nature. In dense QCD, the effective strength of $S U(3)$-flavor breaking effects due to unequal quark masses depends on the mass differences relative to $\mu_{B}$. At sufficiently large $\mu_{B}$, or high density, $S U$ (3) flavor breaking effects are negligible and one is always in the so-called "color-flavor-locked" (CFL) regime, described below. However, when the strange quark mass is made large enough compared to the light quark mass scale, one can show reliably that at intermediate values of $\mu_{B}$ the theory lies in a different regime called 2SC. The 2SC regime is known to be separated by phase transitions from both nuclear matter and high density CFL regimes, because the realizations of global symmetries in the 2SC phase differ from those in both confined nuclear matter and the CFL phase $[26,49]$. The open issue is to understand what happens to the phase structure of QCD near the $S U(3)$ flavor limit.

Let us briefly review what is known about the behavior of the $S U(3)$ flavor symmetric QCD as a function of $\mu_{B}$. There is a critical value of $\mu_{B}$, which we denote by $\mu_{B}^{\text {sat }}$, at which the baryon number density $n_{B}$ jumps from zero to a finite value known as the nuclear saturation density, $n_{B}^{\text {sat }}$. ${ }^{21}$ For $\mu_{B}$ above but close to $\mu_{B}^{\text {sat }}$, the ground state of QCD may be thought of as modestly compressed nuclear matter, by which we mean that a description in terms of interacting nucleon quasiparticles is useful. It is believed that $U(1)_{B}$ is spontaneously broken for any $\mu_{B}>\mu_{B}^{\text {sat }}$ due to condensation of dibaryons, so $S U(3)$-symmetric nuclear matter is a superfluid (see, e.g., Refs. [50,51]). In real nuclear matter neutron pairs condense, while in $S U(3)$ symmetric QCD it is flavor singlet $H$ dibaryons that condense. Nuclear matter should be regarded as a "confined phase" of QCD, with quark confinement defined in the same heuristic fashion as at zero density. (The infamous difficulties of making the notion of confinement precise in theories such as QCD are reviewed in, e.g., Ref. [52].)

In contrast, when $\mu_{B} \gg \mu_{B}^{\text {sat }}$ it becomes natural to describe the system in terms of interacting quarks rather than interacting nucleons. Cold high density quark matter is known to feature "color superconductivity." Attractive gluon mediated interactions between quarks near the Fermi surface lead to quark pairing and condensation, analogous to phonon-induced Cooper pairing of electrons in conventional superconductors. The condensing diquarks in $S U(3)$ flavor-symmetric three-color QCD have the quantum numbers of color-antifundamental scalar fields with charge $2 / 3$ under $U(1)_{B}$. The condensation of these diquark fields spontaneously breaks $U(1)_{B}$ to $\mathbb{Z}_{2}$. At the same time, the color $S U(3)$ gauge group is completely Higgsed, while the flavor $S U(3)$ symmetry is unbroken. The unbroken symmetry transformations consist of common global $S U(3)$ rotations in color and flavor space, and as a result the high density regime of three-flavor QCD is called the CFL phase. The term "color superconductivity" for this phase is something of a misnomer as there are no physically observable macroscopic persistent currents or related phenomena analogous to those present in real superconductors. It is far better to think of this phase as a baryon superfluid in which the $S U(3)$ gauge field is fully Higgsed.

Consequently, as $\mu_{B}$ is increased from $\mu_{B}^{\text {sat }}$ to values that are very large compared to $\max \left(\Lambda_{\mathrm{QCD}}, m_{q}\right)$, the ground state of flavor symmetric QCD evolves from a confining regime with spontaneously broken baryon number symmetry to a Higgs regime which also has spontaneously broken $U(1)_{B}$. The realization of all conventional global symmetries is identical between the low and high density

\footnotetext{
${ }^{21}$ For physical values of quark masses, $n_{B}^{\text {sat }} \sim 0.17 \mathrm{fm}^{-3}$ and $\mu_{B}^{\mathrm{sat}} \sim 920 \mathrm{MeV}$.
} 
regimes. One may also confirm that 't Hooft anomalies match and the pattern of low energy excitations in the different regimes may be smoothly connected $[24,53,54]$.

So a natural question is whether there is a phase transition between the nuclear matter and quark matter regimes of flavor-symmetric QCD [24]. If one can argue that such a phase transition is required, then "confined" nuclear matter and "Higgsed" or "deconfined" quark matter become sharply distinct phases of QCD, and one would obtain some insight into the meaning of the loosely defined term "confinement" in QCD.

This question was the subject of the well-known conjecture by Schäfer and Wilczek [24]. Based on the matching symmetry realizations and other points noted above, they argued that no phase transition is required between the Higgsed (quark matter) and confined (nuclear matter) regimes of $S U(3)$ flavor symmetric QCD, a conjecture known as "quark-hadron continuity." It should be noted that this conjecture is more general than its name suggests. The arguments in favor of this conjecture do not rely on the existence of fermionic fundamental representation matter fields, and apply just as well to gauge theories with fundamental scalar fields and analogous symmetry structures. The Schäfer-Wilczek conjecture can be summarized as the statement that if one considers a gauge theory with gauge group $G$, fundamental representation matter, a $U(1)$ global symmetry, and parameters that allow one to interpolate between a "confining" regime where the $U(1)$ global symmetry is spontaneously broken, and a regime where the gauge group $G$ is completely Higgsed and the $U(1)$ global symmetry is also spontaneously broken, then these regimes are smoothly connected (i.e., portions of a single phase) at zero temperature. ${ }^{22}$ Apart from its intrinsic theoretical interest, the status of quark-hadron continuity is also of experimental interest, at least to the extent that the flavor symmetric limit of QCD is a decent approximation to QCD with physical quark masses. If phase transitions between nuclear matter and quark matter do occur, then the interiors of neutron stars may reach densities where the equation of state and transport properties are strongly affected by such transitions, leading to signatures that might be detectable via multimessenger observations of neutron stars [55-74].

\section{A. Status of the Schäfer-Wilczek conjecture}

In the two decades since Shäfer and Wilczek hypothesized quark-hadron continuity in flavor symmetric QCD, based on compatible symmetry realizations and other necessary but not sufficient correspondences, their

\footnotetext{
${ }^{22}$ This may sound similar to the Fradkin-Shenker-BanksRabinovici theorem $[3,4]$ but, as discussed in the Introduction, the Fradkin-Shenker-Banks-Rabinovici theorem does not apply in situations where the Higgs field is charged under global symmetries, while the Schäfer-Wilczek conjecture concerns precisely such situations.
}

conjecture has reached the status of a highly plausible folk theorem. The expectation of quark-hadron continuity has been used as the starting point for a large number of further conjectures and developments (see, e.g., Refs. [67,75-89]).

Recently, however, three of the present authors argued that a change in particle-vortex statistics between the Higgs regime (quark matter) and the confined regime (nuclear matter) should be interpreted as compelling evidence for invalidity of the Schäfer-Wilczek conjecture $[23] .{ }^{23} \mathrm{We}$ showed that color holonomies around minimal circulation $U(1)_{B}$ vortices have nontrivial phases of $\pm 2 \pi / 3$ in high density quark matter, noted that these holonomies should have vanishing phases in the nuclear matter regime, and used this sharp change in the physics of topological excitations to argue that the nuclear matter and quark matter regimes of dense QCD will be separated by a phase transition.

Subsequent work by other authors [80,94] offered some objections to the arguments in our Ref. [23]. Let us address these objections, starting with Ref. [80] by Hirono and Tanizaki. Changes in particle-vortex statistics are a commonly used diagnostic for phase transitions in gapped phases of matter (see, e.g., Refs. [95,96]). In gapped phases, changes in particle-vortex statistics are connected to changes in intrinsic topological order, which in turn can be related to changes in the realization of higher-form global symmetries [19]. The authors of Ref. [80] tacitly assumed that these statements also hold in gapless systems, and misinterpreted our work [23] as proposing that the zero temperature high density phase of QCD is topologically ordered. The authors of Ref. [80] then argued that this is not the case by discussing the realization of a putative lowenergy "emergent" higher-form symmetry in a gauge-fixed version of $N_{c}=3$ Yang-Mills theory coupled to fundamental Higgs scalar fields. Besides relying on a nonmanifestly gauge invariant approximate description to suggest some higher form symmetry, this discussion missed the central points of Ref. [23] for two reasons. First, Ref. [23] already explicitly emphasized that the CFL phase of QCD is not topologically ordered according to the standard definition of that term, so arguing that the CFL phase does not have topological order in no way contradicts the analysis of Ref. [23]. Second, while Ref. [80] agreed with us that in the flavor-symmetric limit, CFL quark matter features nontrivial color holonomies around $U(1)_{B}$ vortices, it did not address the key question of how this could be consistent with the expected behavior of color holonomies in the nuclear matter regime. Without addressing this crucial question, one cannot conclude that quarkhadron continuity remains a viable scenario in QCD.

Reference [94] by Alford, Baym, Fukushima, Hatsuda, and Tachibana accepted the main result of Ref. [23],

\footnotetext{
${ }^{23}$ For other examinations of vortices in dense quark matter, see also Refs. [33,34,90-93].
} 
namely that in the flavor-symmetric limit color holonomies around vortices take sharply different values in the nuclear matter and quark matter phases. But Ref. [94] argued that the hadronic and color-superconducting regimes may nevertheless be smoothly connected. Alford et al. considered (straight) minimal-circulation vortices in a setting where the density varies along the direction of a vortex, and argued that a single superfluid vortex in the colorsuperconducting regime can connect to a single superfluid vortex in the hadronic regime. ${ }^{24}$ This was interpreted as evidence against a "boojum" 25 of the sort discussed in Refs. [90,92] in which three vortices in the quark matter phase must join in order to pass into the hadronic phase. We agree that there is no reason for a boojum at the interface between quark matter and nuclear matter to necessarily involve multiple vortices joining together. Instead, given the behavior of the color holonomy, it is entirely consistent for the interface to be a genuine boundary between distinct thermodynamic phases, with minimal-energy boojums involving just one minimal circulation vortex on either side, with the behavior of the color gauge fields changing sharply at the interface.

In our view, the key limitations of our work in Ref. [23] were that we could not explicitly compute expectation values of color holonomies in the superfluid nuclear matter regime and demonstrate that they have trivial phases, nor could we give a proof that a change in the behavior of gauge field holonomies around vortices must be associated with a bulk thermodynamic phase transition. (However, we did give physical arguments for this, which we believe are convincing.)

In the preceding sections of the present paper, we have analyzed a 3D model which was deliberately constructed to be analogous to dense QCD, and to which Schäfer and Wilczek's continuity conjecture applies and predicts that no phase transition separates the $U(1)_{\mathrm{G}}$-broken Higgs and confining regimes. This allowed us to examine both of these earlier limitations in the context of this instructive model, and find that continuity does not hold. The Higgs and confining $U(1)_{\mathrm{G}}$-broken regimes of the $3 \mathrm{D}$ theory are distinct phases of matter characterized by a novel order parameter.

\section{B. Higgs versus confinement in $4 \mathrm{D}$ gauge theory}

In earlier sections we focused on our 3D Abelian model because this provided the simplest setting in which to

\footnotetext{
${ }^{24}$ The argument for this statement in Ref. [94] is very simple: one can consider a gedanken situation involving a rotating bucket of density stratified quark/nuclear matter when the quantized superfluid circulation equals unity on every cross section of the bucket. There must then be a single minimal circulation vortex threading both phases and crossing the interface between them.

${ }^{25} \mathrm{~A}$ boojum is a junction or special defect at points where vortices pass through the interface between distinct superfluid phases.
}

examine the issue of Higgs-confinement continuity within superfluid [or spontaneously broken $U(1)$ ] phases, with good theoretical control in both regimes. It is, of course, of interest to understand how the relevant physics might change when one turns to $4 \mathrm{D}$ gauge theories which are more QCD-like.

To that end, we now consider an $S U(3)$ gauge theory coupled to three antifundamental representation scalar fields, as well as an additional gauge-neutral complex scalar field $\phi_{0}$. We will build a model with $S U(3)$ flavor symmetry and write the charged scalar fields as a $3 \times 3$ matrix $\Phi$ which transforms in the bifundamental representation of $S U(3)_{\text {flavor }} \times S U(3)_{\text {gauge }}$,

$$
\Phi \rightarrow F \Phi C^{\dagger}, \quad F \in S U(3)_{\text {flavor }}, \quad C \in S U(3)_{\text {gauge }} .
$$

We also assume the theory has a $U(1)$ global symmetry, which acts as

$$
U(1)_{\mathrm{G}}: \Phi \rightarrow e^{2 i \alpha / 3} \Phi, \quad \phi_{0} \rightarrow e^{2 i \alpha} \phi_{0},
$$

and assume that there exist (or could exist) heavy "baryon" test particles with unit charge under the $U(1)_{\mathrm{G}}$ global symmetry. Since $U(1)_{\mathrm{G}}$ phase rotations which lie within $\mathbb{Z}_{3}$ coincide with the action of $S U(3)$ gauge transformations, the faithfully acting $U(1)$ global symmetry is $U(1)_{\mathrm{G}} / \mathbb{Z}_{3}$.

The action defining this model is given by

$$
\begin{aligned}
S= & \int d^{4} x\left[\frac{1}{2 g^{2}} \operatorname{tr} F_{\mu \nu}^{2}+\operatorname{tr}\left(D_{\mu} \Phi\right)^{\dagger} D^{\mu} \Phi+\left|\partial_{\mu} \phi_{0}\right|^{2}\right. \\
& +m_{\Phi}^{2} \operatorname{tr} \Phi^{\dagger} \Phi+m_{0}^{2}\left|\phi_{0}\right|^{2}+\lambda_{0}\left|\phi_{0}\right|^{4}+\lambda_{\Phi} \operatorname{tr}\left(\Phi^{\dagger} \Phi\right)^{2} \\
& \left.+\epsilon\left(\phi_{0}^{\dagger} \operatorname{det} \Phi+\text { H.c. }\right)+\cdots\right] .
\end{aligned}
$$

As usual, $D_{\mu} \Phi=\partial_{\mu} \Phi+i \Phi A_{\mu}$ is the covariant derivative in the antifundamental representation, and the ellipsis denotes possible further scalar self-interactions which are invariant under the chosen symmetries. The field strength $F_{\mu \nu} \equiv F_{\mu \nu}^{a} t^{a}$, with Hermitian $S U(3)$ generators satisfying $\operatorname{tr} t^{a} t^{b}=\frac{1}{2} \delta^{a b}$.

This $4 \mathrm{D}$ model is very similar to the scalar part of the effective field theory that describes high-density three-color QCD in the CFL quark matter regime [26], with $U(1)_{\mathrm{G}} / \mathbb{Z}_{3}$ playing the role of $U(1)_{B}$ in QCD. The matrix-valued scalar $\Phi$ represents three color-antifundamental diquark fields, so that $\operatorname{det} \Phi$ has the quantum numbers of flavor-singlet dibaryons, which are condensed in both the CFL phase and the $S U(3)$-symmetric nuclear matter phases. Due to the $\epsilon$ coupling between the gauge-neutral scalar $\phi_{0}$ and $\operatorname{det} \Phi$, one can think of $\phi_{0}^{\dagger}$ as a (dynamical) source for flavorsinglet dibaryons. Explicitly introducing the neutral scalar $\phi_{0}$ allows the model (4.3) to describe both the Higgs regime 
and a regime where dibaryons are light, but the gauge and charged scalar fields can be integrated out.

Of course, the effective action for dense QCD in the CFL regime is rotation-invariant but not Lorentz invariant, and also includes heavy fermionic excitations, in contrast to the purely bosonic Lorentz-invariant theory defined by Eq. (4.3). These differences are not relevant to our discussion, and we expect the phase structure of the model (4.3) to mimic the phase structure of QCD with approximate $S U(3)$ flavor symmetry.

Consider the Higgs regime of the model (4.3) where (in gauge-fixed language) $\Phi$ has an expectation value of color-flavor locked form, $\langle\Phi\rangle=v_{\Phi} \mathbf{1}_{3}$, and there is a residual unbroken $S U(3)_{\text {global }}$ symmetry acting as $\Phi \rightarrow U \Phi U^{\dagger}$, with $U \in S U(3)$. The $U(1)_{\mathrm{G}}$ global symmetry is spontaneously broken implying, as always, the existence of vortex topological excitations. To describe a straight "superfluid" vortex, using cylindrical coordinates with $r=0$ at the center of the vortex, one may fix a gauge in which the vortex configuration has $\Phi$ diagonal and $A_{\mu}$ taking values in the Cartan subalgebra,

$$
\begin{aligned}
& \phi_{0}(r, \theta)=v_{0} f_{0}(r) e^{i k \theta}, \\
& \Phi(r, \theta)=v_{\Phi} \operatorname{diag}\left(f_{1}(r) e^{i(n+k) \theta}, f_{2}(r) e^{i(m-n) \theta}, f_{3}(r) e^{-i m \theta}\right),
\end{aligned}
$$

$$
A_{\theta}(r)=\frac{a h_{8}(r)}{2 \pi r} t_{8}+\frac{b h_{3}(r)}{2 \pi r} t_{3} .
$$

Here $k, m, n \in \mathbb{Z}$, with $k$ the vortex winding number, $t_{8} \equiv$ $\frac{1}{2 \sqrt{3}} \operatorname{diag}(1,1,-2)$ and $t_{3} \equiv \frac{1}{2} \operatorname{diag}(1,-1,0)$ are the usual diagonal $S U(3)$ generator matrices, and the radial profile functions $\left\{f_{i}\right\}$ and $\left\{h_{i}\right\}$ approach 1 as $r \rightarrow \infty$. Minimizing the long-distance energy density of the vortex configuration determines the gauge field asymptotics. One finds

$a=-\frac{2 \pi}{\sqrt{3}}(k+3 m), \quad b=-2 \pi(k+2 n-m)$.

The minimal energy vortex with unit circulation $(k=1)$ corresponds to $n=m=0$ (with physically equivalent forms related by Weyl reflections), in which case

$$
a=-\frac{2 \pi}{\sqrt{3}}, b=-2 \pi,
$$

and

$$
\begin{aligned}
\Phi(r, \theta) & =v_{\Phi} \operatorname{diag}\left(f(r) e^{i \theta}, g(r), g(r)\right), \\
A_{\theta}(r) & =\frac{h(r)}{3 r} \operatorname{diag}(-2,1,1) .
\end{aligned}
$$

Here, we have set $f_{1}(r)=f(r), f_{2}(r)=f_{3}(r)=g(r)$, and $h_{3}(r)=h_{8}(r)=h(r)$. The minimal-energy vortex configuration (4.7) preserves an $S U(2) \times U(1)$ symmetry (cf. Ref. [97]). Hence, these minimal energy unit-winding vortices have zero modes associated with the moduli space

$$
\frac{S U(3)}{S U(2) \times U(1)}=\mathbb{C P}^{2} .
$$

Consequently, the world sheet effective field theory for a vortex contains a $\mathbb{C P}^{2}$ nonlinear sigma model $[34,36,98]$. But the $\mathbb{C P}^{2}$ model in two spacetime dimensions (with vanishing topological angle $\theta$ ) has a mass gap and a unique ground state angle (see e.g., Refs. [99,100]). So, despite the appearance of the classical configuration (4.4), the $S U(3)_{\text {global }}$ symmetry is unbroken both in the vacuum and in the presence of vortices.

Now consider the behavior of our vortex holonomy order parameter in this theory. The gauge field holonomy is now a path-ordered exponential around some contour $C$, $\Omega(C) \equiv \mathcal{P}\left(e^{i \int_{C} A}\right)$, and defines an $S U(3)$ group element. The natural non-Abelian version of our vortex order parameter involves gauge invariant traces of holonomies,

$$
O_{\Omega} \equiv \lim _{r \rightarrow \infty} \frac{\langle\operatorname{tr} \Omega(C)\rangle_{1}}{\langle\operatorname{tr} \Omega(C)\rangle}
$$

where in the numerator the circular contour $C$ encircles a minimal vortex in the same direction as the circulation of the $U(1)_{\mathrm{G}}$ current. ${ }^{26}$ Both expectations in the ratio (4.9) have perimeter-law dependence on the size of the contour $C$ arising from quantum fluctuations on scales small compared to $r$, but this geometric factor cancels by construction in the ratio. Unbroken charge conjugation symmetry implies that the denominator is real, and it must be positive throughout any phase connected to a weakly coupled regime. So as in our earlier Abelian model, the behavior of $O_{\Omega}$ is determined by the phase of the vortex expectation value in the numerator.

A trivial calculation (identical to that in Ref. [23]) shows that at tree level, far from the vortex,

$$
\frac{1}{3}\langle\operatorname{tr} \Omega(C)\rangle_{1}^{\text {tree }}=e^{2 \pi i / 3} .
$$

demonstrating that $O_{\Omega}=e^{2 \pi i / 3}$ at tree level. An effective field theory argument, analogous to that given in Sec. III B (see also Appendix B), shows that this result is unchanged when quantum fluctuations are taken into account, as long as they are not so large as to restore the spontaneously

\footnotetext{
${ }^{26}$ Once again, the numerator is defined by a constrained functional integral with a prescribed vortex world sheet, with the size of that world sheet and the minimal separation between the vortex world-sheet and the holonomy contour $C$ scaling together as the contour radius $r$ increases.
} 
broken $U(1)_{\mathrm{G}}$ symmetry. To see this, consider the form of the effective action generated by integrating out fluctuations on scales small compared to $r$. Only terms in the effective action with two derivatives acting on the charged scalar field $\Phi$ can contribute to the $\mathcal{O}\left(1 / r^{2}\right)$ holonomydependent part of the energy density, and hence affect the gauge field asymptotics (4.6) which determines the expectation value of holonomies far from the vortex core. Traces of operators containing a single covariant derivative, such as $\operatorname{Tr} \Phi^{\dagger} D_{\mu} \Phi$, are independent of the gauge field far from the vortex core. Consequently, the portion of the effective action which controls the holonomy expectation value far from a vortex may be written in the form

$$
\begin{aligned}
& S_{\text {eff, } S U(3) \text { holonomy }} \\
& =\int d^{4} x\left\{\operatorname{Tr}\left[f_{1}\left(\phi_{0}, \Phi\right)\left(D_{\mu} \Phi\right)^{\dagger} f_{2}\left(\phi_{0}, \Phi\right)\left(D^{\mu} \Phi\right)\right]\right. \\
& \left.\quad+\epsilon^{A B C} \epsilon_{I J K} f_{3}\left(\phi_{0}, \Phi\right)_{A}^{I}\left(D_{\mu} \Phi\right)_{B}^{J}\left(D^{\mu} \Phi\right)_{C}^{K}\right\}+ \text { H.c., }
\end{aligned}
$$

where $A, B, C$ are color indices and $I, J, K$ are flavor indices. The three coefficient functions $\left\{f_{i}\right\}$ depend on the fields $\phi_{0}$ and $\Phi$, but not on their derivatives, only in combinations which are invariant under $U(1)_{\mathrm{G}}$. The function $f_{1}$ is a color adjoint and flavor singlet (such as $\Phi^{\dagger} \Phi$ ), $f_{2}$ is color singlet and flavor adjoint (such as $\Phi \Phi^{\dagger}$ ), and $f_{3}$ is antifundamental in color and fundamental in flavor (such as $\left.\phi_{0}^{\dagger} \Phi\right)$. Plugging in the configuration (4.4), one can easily verify that both terms in (4.11) have extrema, with respect to the asymptotic gauge field coefficients $a$ and $b$, at the same location (4.6) regardless of the form of the functions $\left\{f_{i}\right\}$. Therefore small quantum corrections do not perturb the gauge field asymptotics far from a vortex, and hence cannot shift the phase of the vortex holonomy expectation $\langle\operatorname{tr} \Omega(C)\rangle_{1}$ away from $2 \pi / 3$. Hence, we learn that

$$
U(1)_{\mathrm{G}} \text {-broken Higgs phase: } O_{\Omega}=e^{2 \pi i / 3}
$$

holds exactly throughout the phase connected to the weakly coupled Higgs regime.

Alternatively, when $m_{\Phi}^{2} \gtrsim \Lambda^{2}$, with $\Lambda$ the strong dynamics scale of the theory, we can recycle the arguments of Sec. III E to understand the behavior of $O_{\Omega}$. In this regime, due to the presence of heavy dynamical charged excitations, the expectation values of large fundamental representation Wilson loops are (exponentially) dominated by a perimeter-law contribution. Physically, a Wilson loop describes a process where a fundamental representation test particle and antiparticle are inserted at some point, separated and then recombined as they traverse the contour $C$. The perimeter law behavior arises from configurations in which dynamical fundamental representation excitations of mass $m_{\Phi}$ are pair-created and dress the test charge and anticharge to create two bound gauge-neutral "mesons." These mesons have a physical size of order $\ell_{\text {meson }} \sim$ $\min \left(\Lambda^{-1},\left(\alpha_{s} m_{\Phi}\right)^{-1}\right)$, and experience no long range interactions. Once the Wilson loop size exceeds the string breaking scale $\sim 2 m_{\Phi} / \Lambda^{2}$, pair creation of dynamical charges of mass $m_{\Phi}$ and the associated meson formation becomes the dominant process contributing to fundamental Wilson loop expectation values.

The perimeter law contribution to large fundamental representation Wilson loop expectation values arises from fluctuations of the gauge-charged fields within distances of order of $\ell_{\text {meson }}$ from any point on the contour $C$. The amplitude for such screening fluctuations, and consequent meson formation, must be completely insensitive to the presence of a vortex very far away at the center of the loop. This means that the holonomy expectations in the numerator and denominator of the vortex observable (4.9) will be identical (up to exponentially small corrections vanishing as $r \rightarrow \infty$ ), leading to the conclusion that

$$
U(1)_{\mathrm{G}} \text {-broken confining phase: } O_{\Omega}=1 \text {. }
$$

Once again, the differing results (4.12) and (4.13), each strictly constant within their respective domains, implies that $O_{\Omega}$ cannot be a real-analytic function of $m_{\Phi}^{2}$. Adapting the arguments in Sec. III D regarding the impact of abrupt changes in the properties of vortex loops on the ground state energy, we see that $O_{\Omega}$ functions as an order parameter that distinguishes the $U(1)_{\mathrm{G}}$-broken Higgs and $U(1)_{\mathrm{G}^{-}}$ broken confining phases of this four-dimensional $S U(3)$ gauge theory with $S U(3)$ flavor symmetry. ${ }^{27}$

Finally, if the $S U(3)$ flavor symmetry of this theory is explicitly broken by a small perturbation, a simple generalization of the analysis leading to the gauge field asymptotics (4.7) implies that the phase of $O_{\Omega}$ will now deviate

\footnotetext{
${ }^{27}$ Further evidence that changes in our nonlocal order parameter signal genuine phase transitions in non-Abelian gauge theories may be gained by considering other calculable examples. One such case is described in Appendix B. A different example which is closer to the model discussed in this section consists of a version of the theory (4.3) in three spacetime dimensions, with gauge group $S U(2)$ and two flavors of $S U(2)$ antifundamental scalar fields, with a global flavor symmetry containing an $S U(2)$ factor. Generalizing the analysis in Sec. III D to this non-Abelian model, we have checked that there is a set of parameters (essentially identical to the ones in Sec. III D) for which the phase transition between the $U(1)_{\mathrm{G}}$-broken confining and Higgs regimes is strongly first order as a function of the mass of the antifundamental scalars. The fact that the transition is strongly first order allows the existence of the phase transition to be reliably established despite the fact that the gauge sector is strongly coupled within the $U(1)_{\mathrm{G}}$-broken confining phase. It is easy to check in this example that our vortex observable $O_{\Omega}$ jumps from +1 to -1 across the transition, and serves as an order parameter distinguishing distinct phases, even when the transition is no longer strongly first order. Finally, it is easy to check that these statements generalize to $N=N_{f}>2$ gauge theories.
} 
slightly from $2 \pi / 3$. But in the $U(1)_{\mathrm{G}}$-broken confined phase, $O_{\Omega}$ remains exactly 1 due to the confinement and string breaking effects discussed above. This implies that the $U(1)_{\mathrm{G}}$-broken Higgs and $U(1)_{\mathrm{G}}$-broken confining regimes of our 4D $S U(3)$ scalar theory (4.3) must remain separated by a quantum phase transition even when the $S U$ (3) flavor symmetry is explicitly broken. Most importantly, essentially the same argument applies to dense QCD.

Before leaving this section, we note that one may consider our original 3D model (2.4), or the 4D nonAbelian generalization (4.3), with the addition of a nonzero chemical potential for the $U(1)_{\mathrm{G}}$ symmetry. Such a chemical potential explicitly breaks charge conjugation symmetry, just as the baryon chemical potential in dense QCD. In our earlier discussion we used unbroken charge conjugation symmetry to conclude that the ground state expectation value of the holonomy must be real. But, as noted in footnote 10, for a reflection-symmetric holonomy contour (such as a circle), reflection symmetry is an equally good substitute. Consequently, all of our arguments demonstrating that the phase of the holonomy encircling a vortex at large distance serves as an order parameter distinguishing "confining" and "Higgs" superfluid phases go through without modification in the presence of a nonzero chemical potential.

In summary, we have shown that consideration of our new order parameter implies that there is a phase transition between nuclear matter and quark matter in dense QCD near the $S U(3)$ flavor limit. This means that the confining nuclear matter regime of QCD [at least with approximate $S U$ (3) flavor symmetry] has a sharp definition as a phase of QCD where the expectation values of color holonomies around superfluid vortices are positive, while quark matter - a Higgs regime - can be defined as the phase of QCD where these holonomy expectation values become complex. Given the notorious difficulties in giving a sharp definition for confining and Higgs regimes in gauge theories with fundamental representation matter (see Ref. [52] for a review), this is a satisfying result in the theory of strong interactions. Our results are also encouraging for observational searches for evidence of quark matter cores in neutron stars (see, e.g., Refs. [57-59,6172]), because our results imply that hadronic matter and quark matter must be separated by a phase transition as a function of density.

\section{CONCLUSIONS}

We have explored the phase structure of gauge theories with fundamental representation matter fields and a $U(1)$ global symmetry. Motivated by the physics of dense QCD, we considered both Higgs and confining portions of the phase diagram in which the $U(1)$ global symmetry is spontaneously broken, and hence the theory is gapless due to the presence of a Nambu-Goldstone boson. These two regimes cannot be distinguished by conventional local order parameters probing global symmetry realizations, nor do they naturally fit into more modern classification schemes based on topological order and related concepts. Nevertheless, using a novel vortex order parameter introduced in Sec. III, we found that $U(1)$-broken confining and Higgs regimes are sharply distinct phases of matter separated by at least one phase transition in parameter space, as illustrated in Fig. 1. In Secs. II and III (and Appendix B) we examined instructive parity-invariant Abelian (and non-Abelian) gauge theories in three spacetime dimensions illustrating this physics. Then in Sec. IV we considered related theories with a $U(1)$ global symmetry in four spacetime dimensions and explained how our considerations serve to rule out the Schäfer-Wilczek conjecture of quark-hadron continuity in cold dense QCD.

Why are these results interesting? First, we have added to the toolkit of techniques for diagnosing phase transitions in gauge theories and shown that it predicts previously unexpected phase transitions in theories with fundamental representation matter fields. Second, our analysis implies a phase transition between quark matter and nuclear matter in dense QCD near the $S U(3)$ flavor limit, with possible implications for observable properties of neutron stars. Third, our analysis provides a sharp distinction between a confined nuclear matter regime of QCD and dense quark matter. In other words, it provides sharp answers to some basic questions about strong dynamics:

(i) "What is the confined phase of QCD?" Our work shows that this question has a sharp answer when the $U(1)_{B}$ baryon number symmetry is spontaneously broken. The confined phase of QCD with spontaneously broken $U(1)_{B}$ symmetry can be defined as the phase of QCD where the expectation values of color holonomies around minimal-circulation superfluid vortices are positive.

(ii) "What is cold quark matter?" Our analysis shows that cold quark matter can be defined as the phase of QCD where the expectation values of color holonomies around minimal-circulation superfluid vortices have nonvanishing phases.

Our results raise a number of other interesting questions that we hope can be addressed in future work. These include:

(i) What is the nature of the point in Fig. 1 where the three different phase transition curves intersect?

(ii) What can be said in general about the order of the phase transition(s) separating $U(1)$-broken confining and Higgs phases in the theories we have considered? As discussed in Sec. III D, for some ranges of parameters there is a single first order phase transition. Is this always the case, or is there a range of parameters where the transition becomes second order? How does the answer depend on the spacetime dimension? These issues are of more than just theoretical interest, because the properties of the nuclear to quark matter phase transition(s) in dense 
QCD can have observational impacts for the physics of neutron stars.

(iii) Relatedly, when the transition is first order, what is the physics on an interface separating coexisting phases? This is also directly connected to potential neutron star phenomenology.

(iv) What happens to the phase structure of the class of theories we have considered, in both three and four spacetime dimensions, at nonzero temperature?

(v) How should the modern classification of the phases of matter be generalized when considering transitions between gapless regimes? Is there a natural embedding of the constructions in this paper into some more general framework? In Appendix $\mathrm{C}$ we gauge the $U(1)_{\mathrm{G}}$ symmetry of our 3D Abelian model and show that the resulting gapped theory [which flows to topological quantum field theories (TQFTs) at long distances] has a phase transition analogous to the Higgs-confinement phase transition studied in the body in the paper. But we also argue that, by itself, this cannot be used to infer the existence of a phase transition in the original model with a global $U(1)_{\mathrm{G}}$ symmetry.

(vi) Can our construction be generalized to gauge theories where the $U(1)_{\mathrm{G}}$ global symmetry is explicitly broken to a discrete subgroup $\mathbb{Z}_{k}$ ? Such theories would contain domain walls, and the behavior of gauge field holonomies around domain wall junctions could be used to identify phase transitions.

(vii) Are there condensed matter systems which realize the physics of $U(1)$-broken Higgs-confinement phase transitions?

\section{ACKNOWLEDGMENTS}

We are especially grateful to Fiona Burnell for extensive discussions and collaboration at the initial stages of this project. We are also grateful to M. Alford, F. Benini, S. Benvenuti, K. S. Damle, L. Fidkowski, D. Harlow, Z. Komargodski, S. Minwalla, E. Poppitz, N. Seiberg, Y. Tanizaki, and M. Ünsal for helpful discussions and suggestions during the long gestation of this paper. A. C. acknowledges support from the University of Minnesota. T. J. is supported by a UMN CSE Fellowship. S. S. acknowledges the support of Iowa State University startup funds. L.Y. acknowledges support from the U.S. Department of Energy Grant No. DE-SC-0011637.

\section{APPENDIX A: HIGGS PHASE VORTEX PROFILE FUNCTIONS}

Recall that the vortex configuration in our 3D model has the form given by Eqs. (3.4) and (3.7), repeated here:

$$
\phi_{0}=v_{0} f_{0}(r) e^{i k \theta}, \quad \phi_{+}=v_{c} f_{+}(r) e^{i(n-k) \theta}, \quad \phi_{-}=v_{c} f_{-}(r) e^{-i n \theta}, \quad A_{\theta}=\frac{\Phi h(r)}{2 \pi r},
$$

with the radial profile functions $h, f_{ \pm}$, and $f_{0}$ all approaching 1 as $r \rightarrow \infty$. The equation of motion for the gauge field profile $h(r)$ is

$$
\frac{\Phi}{2 \pi}\left(\frac{d^{2} h}{d r^{2}}-\frac{1}{r} \frac{d h}{d r}\right)=-2 e^{2} v_{c}^{2}\left[\left(n-k-\frac{\Phi h}{2 \pi}\right) f_{+}^{2}+\left(n-\frac{\Phi h}{2 \pi}\right) f_{-}^{2}\right]
$$

while the scalar field profile functions obey

$$
\begin{aligned}
{\left[\frac{d^{2} f_{+}}{d r^{2}}+\frac{1}{r} \frac{d f_{+}}{d r}-\frac{f_{+}}{r^{2}}\left(n-k-\frac{\Phi h}{2 \pi}\right)^{2}\right] } & =-\left|m_{c}^{2}\right| f_{+}+2 \lambda_{c} v_{c}^{2} f_{+}^{3}-\epsilon v_{0} f_{-} f_{0}, \\
{\left[\frac{d^{2} f_{-}}{d r^{2}}+\frac{1}{r} \frac{d f_{-}}{d r}-\frac{f_{-}}{r^{2}}\left(n-\frac{\Phi h}{2 \pi}\right)^{2}\right] } & =-\left|m_{c}^{2}\right| f_{-}+2 \lambda_{c} v_{c}^{2} f_{-}^{3}-\epsilon v_{0} f_{+} f_{0}, \\
{\left[\frac{d^{2} f_{0}}{d r^{2}}+\frac{1}{r} \frac{d f_{0}}{d r}-\frac{f_{0}}{r^{2}}(k)^{2}\right] } & =-\left|m_{0}^{2}\right| f_{0}+2 \lambda_{0} v_{0}^{2} f_{0}^{3}-\epsilon \frac{v_{c}^{2}}{v_{0}} f_{+} f_{-} .
\end{aligned}
$$

As discussed in Sec. III B [cf. Eq. (3.9)] the minimal energy solution has $\Phi=(2 n-k) \pi$. Inserting this value and examining the resulting large $r$ asymptotic behavior of the profile functions, one finds that $h(r)$ equals 1 up to exponentially falling corrections. This will be demonstrated below. Neglecting such exponentially small terms, the scalar profile functions satisfy

$$
\left[\frac{d^{2} f_{ \pm}}{d r^{2}}+\frac{1}{r} \frac{d f_{ \pm}}{d r}-\frac{k^{2}}{4} \frac{f_{ \pm}}{r^{2}}\right]=-\left|m_{c}^{2}\right| f_{ \pm}+2 \lambda_{c} v_{c}^{2} f_{ \pm}^{3}-\epsilon v_{0} f_{\mp} f_{0}
$$




$$
\left[\frac{d^{2} f_{0}}{d r^{2}}+\frac{1}{r} \frac{d f_{0}}{d r}-k^{2} \frac{f_{0}}{r^{2}}\right]=-\left|m_{0}^{2}\right| f_{0}+2 \lambda_{0} v_{0}^{2} f_{0}^{3}-\epsilon \frac{v_{c}^{2}}{v_{0}} f_{+} f_{-}
$$

Demanding that the scalar profile functions $\left\{f_{i}\right\}$ approach 1 as $r \rightarrow \infty$ and requiring that the resulting right-hand sides of Eq. (A4) vanish determines the condensate magnitudes $v_{0}$ and $v_{c}$. One finds $v_{c}^{2}=\left(\left|m_{c}^{2}\right|+\epsilon v_{0}\right) /\left(2 \lambda_{c}\right)$ with $v_{0}$ the positive solution of the cubic equation

$$
4 \lambda_{0} \lambda_{c} v_{0}^{3}-\left(2 \lambda_{c}\left|m_{0}^{2}\right|+\epsilon^{2}\right) v_{0}-\epsilon\left|m_{c}^{2}\right|=0 .
$$

One may then verify that the resulting scalar profile functions have the asymptotic forms

$$
f_{ \pm}(r)=1-\frac{\ell_{c}^{2}}{r^{2}}+\mathcal{O}\left(r^{-4}\right), \quad f_{0}(r)=1-\frac{\ell_{0}^{2}}{r^{2}}+\mathcal{O}\left(r^{-4}\right)
$$

with

$$
\ell_{c}^{2}=\frac{k^{2}}{4 v_{c}^{2}} \frac{6 \lambda_{0} v_{0}^{2}-\left|m_{0}^{2}\right|+4 \epsilon v_{0}}{2 \lambda_{c}\left(6 \lambda_{0} v_{0}^{2}-\left|m_{0}^{2}\right|\right)-\epsilon^{2}}, \quad \ell_{0}^{2}=\frac{k^{2}}{4 v_{0}} \frac{\epsilon+8 \lambda_{c} v_{0}}{2 \lambda_{c}\left(6 \lambda_{0} v_{0}^{2}-\left|m_{0}^{2}\right|\right)-\epsilon^{2}}
$$

The large $r$ power-law tails in scalar field profile functions are characteristic features of global vortices. But since we are in a Higgs phase, the gauge field should have exponential falloff. To verify this, we parallel the treatment of Ref. [34] and rewrite the coupled equations in terms of sums and differences of the charged field profiles. Let

$$
\begin{aligned}
& h=1+H, \quad f_{+}=1+F+\frac{G}{2}, \\
& f_{0}=1+F_{0}, \quad f_{-}=1+F-\frac{G}{2},
\end{aligned}
$$

and then linearize the field equations in the deviations $F_{0}$, $F, G$, and $H$. The leading behavior of $F$ and $F_{0}$ can be read off from Eq. (A6). The linearized equations for $G$ and $H$ do not involve $F$ or $F_{0}$ and read

$$
\begin{aligned}
{\left[\frac{d^{2}}{d r^{2}}+\frac{1}{r} \frac{d}{d r}-\tilde{m}^{2}-\frac{k^{2}}{4 r^{2}}\right] G } & =\frac{k(2 n-k)}{r^{2}} H, \\
(2 n-k)\left[\frac{d^{2}}{d r^{2}}-\frac{1}{r} \frac{d}{d r}-m_{A}^{2}\right] H & =k m_{A}^{2} G,
\end{aligned}
$$

where $m_{A}^{2} \equiv 4 e^{2} v_{c}^{2}$ and $\tilde{m}^{2} \equiv 4 \lambda_{c} v_{c}^{2}+2 \epsilon v_{0}$. Taking, for simplicity, $k=n=1$, one may check that the two independent homogeneous solutions have the asymptotic forms

$$
\begin{aligned}
G_{\mathrm{I}}(r) & \sim m_{A}^{2}\left(m_{A} r\right)^{-3 / 2} e^{-m_{A} r}, \\
H_{\mathrm{I}}(r) & \sim\left(\tilde{m}^{2}-m_{A}^{2}\right)\left(m_{A} r\right)^{1 / 2} e^{-m_{A} r}, \\
G_{\mathrm{II}}(r) & \sim\left(\tilde{m}^{2}-m_{A}^{2}\right)(\tilde{m} r)^{-1 / 2} e^{-\tilde{m} r}, \\
H_{\mathrm{II}}(r) & \sim m_{A}^{2}(\tilde{m} r)^{-1 / 2} e^{-\tilde{m} r} .
\end{aligned}
$$

The most general solution is a linear combination of solutions I and II. Depending on whether $m_{A}$ or $\tilde{m}$ is smaller, either solution I or solution II dominates at large distance. In either case, the gauge field profile function approaches its asymptotic value far from the vortex exponentially fast.

\section{APPENDIX B: EMBEDDING IN A NON-ABELIAN GAUGE THEORY}

Consider a parity-invariant $S U(2)$ gauge theory containing one real adjoint representation scalar field $\zeta$, one fundamental representation scalar $\Phi$, and one $S U(2)$-singlet complex scalar field $\phi_{0}$. We take the action of the theory to be

$$
\begin{aligned}
S= & \int d^{3} x\left[\frac{1}{2 g^{2}} \operatorname{tr} \mathcal{F}_{\mu \nu}^{2}+\left|D_{\mu} \Phi\right|^{2}+\operatorname{tr}\left(D_{\mu} \zeta\right)^{2}+\left|\partial_{\mu} \phi_{0}\right|^{2}+m_{\Phi}^{2}|\Phi|^{2}+m_{0}^{2}\left|\phi_{0}\right|^{2}\right. \\
& \left.+\lambda_{\Phi}|\Phi|^{4}+\lambda_{0}\left|\phi_{0}\right|^{4}+\lambda_{\zeta} \operatorname{tr}\left(\zeta^{2}-\frac{1}{4} v_{\zeta}^{2}\right)^{2}+\varepsilon\left(\phi_{0} \Phi^{T} i \sigma_{2} \zeta \Phi+\text { H.c. }\right)+\cdots\right]
\end{aligned}
$$


where the covariant derivatives $D_{\mu} \Phi \equiv \partial_{\mu} \Phi-i \mathcal{A}_{\mu} \Phi$ and $D_{\mu} \zeta \equiv \partial_{\mu} \zeta-i\left[\mathcal{A}_{\mu}, \zeta\right]$, the gauge field $\mathcal{A}_{\mu} \equiv \mathcal{A}_{\mu}^{a} t^{a}$, and the $S U(2)$ generators obey $\operatorname{tr} t^{a} t^{b}=\frac{1}{2} \delta^{a b}$. The couplings $\varepsilon, \lambda_{\Phi}$, $\lambda_{0}$, and $\lambda_{\zeta}$ are assumed real and positive, and the ellipsis stands for further scalar potential terms consistent with the symmetries imposed below.

In addition to parity (and gauge and Euclidean invariance), we assume the theory has a $U(1)_{\mathrm{G}}$ global symmetry acting as

$$
U(1)_{\mathrm{G}}: \Phi \rightarrow e^{-i \alpha} \Phi, \quad \phi_{0} \rightarrow e^{2 i \alpha} \phi_{0} .
$$

The $\mathbb{Z}_{2}$ subgroup generated by the $\alpha=\pi$ sign flip is part of the $S U(2)$ gauge symmetry, so the faithfully acting global symmetry is $U(1)_{\mathrm{G}} / \mathbb{Z}_{2}$. We also assume the existence of a discrete global symmetry we will call $\left(\mathbb{Z}_{2}\right)_{\mathrm{F}}$, acting as

$$
\left(\mathbb{Z}_{2}\right)_{\mathrm{F}}:\left(\begin{array}{c}
\zeta \\
\Phi \\
\mathcal{A}_{\mu}
\end{array}\right) \rightarrow\left(\begin{array}{c}
-U_{4} \zeta U_{4}^{\dagger} \\
-i U_{4} \Phi \\
U_{4} \mathcal{A}_{\mu} U_{4}^{\dagger}
\end{array}\right),
$$

where $U_{4} \equiv\left(\begin{array}{ll}0 & i \\ i & 0\end{array}\right) \in S U(2)$. This transformation leaves the action (B1) invariant and acts as a $\mathbb{Z}_{2}$ symmetry on gauge invariant observables.

We consider this model when $v_{\zeta}^{2} \gg g^{2}$, leading to Higgsing of the $S U(2)$ gauge group down to a $U(1)$ Cartan subgroup. Choosing, for convenience, a gauge where $\zeta=v_{\zeta} \sigma_{3} / 2$, one sees that the "color" components $\mathcal{A}^{1}$ and $\mathcal{A}^{2}$ become massive while $\mathcal{A}_{\mu}^{3}$ remains massless. Writing $\Phi \equiv\left(\begin{array}{c}\phi_{+} \\ \phi_{-}\end{array}\right)$, the component fields $\phi_{ \pm}$transform with charge $\pm 1 / 2$ under the unbroken $U(1)$ gauge group. To write the resulting low energy theory, below the scale $m_{W} \equiv g v_{\zeta}$, in the most convenient form let $e \equiv g / 2$ and $A_{\mu} \equiv \frac{1}{2} \mathcal{A}_{\mu}^{3}$. This makes $A_{\mu}$ an Abelian gauge field with coupling $e$ interacting with fields $\phi_{ \pm}$having charges \pm 1 . The final term in the action (B1) with coefficient $\varepsilon$ becomes

$$
\begin{aligned}
& \varepsilon\left(\phi_{0} \Phi^{T} i \sigma_{2} \zeta \Phi+\text { H.c. }\right) \\
& =\varepsilon \phi_{0}\left(\phi_{+}, \phi_{-}\right)\left(\begin{array}{cc}
0 & -v_{\zeta} / 2 \\
-v_{\zeta} / 2 & 0
\end{array}\right)\left(\begin{array}{l}
\phi_{+} \\
\phi_{-}
\end{array}\right)+\text {H.c. } \\
& =-\varepsilon v_{\zeta}\left(\phi_{0} \phi_{+} \phi_{-}+\text {H.c. }\right),
\end{aligned}
$$

after setting $\zeta$ to its expectation value. If one now identifies $\epsilon \equiv \varepsilon v_{\zeta}$ and $m_{c}^{2} \equiv m_{\Phi}^{2}$, then the resulting low-energy description of this non-Abelian model, now involving the fields $\phi_{ \pm}, \phi_{0}$, and $A_{\mu}$, precisely coincides with our original Abelian model (2.4).

Now consider the behavior of the non-Abelian model (B1) as the mass parameters $m_{\Phi}^{2}$ and $m_{0}^{2}$ are varied. To begin, suppose that $m_{\Phi}^{2} \gg g^{2} v_{\zeta}^{2}$, so that the fundamental scalar field $\Phi$ is not condensed. If $m_{0}^{2}$ is sufficiently negative, the neutral scalar $\phi_{0}$ will condense and the $U(1)_{\mathrm{G}}$ symmetry will be spontaneously broken; otherwise $U(1)_{\mathrm{G}}$ will be unbroken. In either case, the $S U(2)$ adjoint Higgs mechanism leads to the existence of stable finiteaction monopole-instantons [101,102] whose stability is guaranteed by $\pi_{2}(S U(2) / U(1))=\mathbb{Z}$. The Abelian magnetic flux (defined on scales large compared to $m_{W}^{-1}$ ) through a spacetime surface $M_{2}$ has an $S U(2)$ gaugeinvariant definition

$$
\Phi_{B}\left(M_{2}\right) \equiv \frac{1}{\left|v_{\zeta}\right|} \int_{M_{2}} \operatorname{tr}(\zeta \mathcal{F}),
$$

where $\mathcal{F}$ is the two-form field strength. We have normalized the flux $\Phi_{B}$ (not to be confused with the field $\Phi$ ) so that when written in terms of the 2-form field strength $F$ of the Abelian gauge field $A_{\mu}$, the flux has the conventional form $\Phi_{B}=\int_{M_{2}} F$. Note that $\Phi_{B}$ is odd under the $\left(\mathbb{Z}_{2}\right)_{\mathrm{F}}$ global symmetry. The minimal magnetic monopole instantons in $S U(2)$ gauge theory have $\Phi_{B}\left(S^{2}\right)= \pm 2 \pi$, where $S^{2}$ is a spacetime two-sphere surrounding the center of the monopole instanton. If the center of the monopole is at $r=0$, then as $r \rightarrow \infty$, at a large distance from the monopole the $S U(2)$ gauge field and adjoint scalar approaches the asymptotic forms

$$
\left(\mathcal{A}^{\mu}\right)^{a} \rightarrow \frac{\epsilon^{a \mu \nu} \hat{r}_{\nu}}{r}, \zeta^{a} \rightarrow v_{\zeta} \hat{r}^{a}
$$

in "hedgehog" gauge, with $\hat{r}_{\mu}$ a radial unit vector. The action of a monopole-instanton has the form

$$
S_{\mathrm{I}}=\frac{4 \pi v_{\zeta}}{g} f\left(\frac{\lambda_{\zeta}}{g^{2}}\right)
$$

where the dimensionless and monotonically increasing function $f$ varies between $f(0)=1$ and $f(\infty)=1.787$ [103-105]. The associated monopole operator, characterizing the effect of a monopole on long distance physics, has the form $e^{i \sigma} e^{-S_{\mathrm{I}}}$, with $\sigma$ the magnetic dual of the Abelian field strength $F$. As discussed in Sec. II, these monopoleinstantons generate a potential of the form $e^{-S_{\mathrm{I}}} \cos (\sigma)$ for the dual photon of $A_{\mu}$, leading to a mass gap for the low energy Abelian gauge field and confinement of heavy test charges.

Alternatively, if $-m_{\Phi}^{2} \gg g^{4}$, then the fundamental representation scalar $\Phi$ will condense, leading to complete Higgsing of the $S U(2)$ gauge symmetry, along with spontaneous breaking of the global $U(1)_{\mathrm{G}}$ symmetry, regardless of the value of $m_{0}^{2}$. Monopole instantons are now confined by magnetic flux tubes and have a negligible effect on long distance physics. All components of the $S U(2)$ gauge field acquire mass via the Higgs mechanism.

The question remains: are the confining (via Polyakov mechanism) and fully Higgsed regimes, both with spontaneous $U(1)_{\mathrm{G}}$ breaking, smoothly connected? All of the 
analysis of Sec. III generalizes in a straightforward fashion to this non-Abelian model and shows that the answer is no. To see this, one may consider the natural generalization of our previous vortex holonomy observable (3.2) which replaces the Abelian gauge field holonomy with the trace of the non-Abelian holonomy, ${ }^{28}$

$$
O_{\Omega}^{S U(2)} \equiv \lim _{r \rightarrow \infty} \frac{\langle\operatorname{tr} \Omega(C)\rangle_{1}}{\langle\operatorname{tr} \Omega(C)\rangle}
$$

Just as in our original Abelian model, the holonomy expectation values in numerator and denominator will have perimeter law decay of their magnitudes, but this size dependence cancels in the ratio by construction. The denominator is guaranteed to be positive in weakly coupled regimes because charge conjugation (or reflection) symmetry requires it to be real, it is positive at tree level, and hence small quantum corrections cannot turn it negative. So the ratio of expectations is determined by the phase of the vortex expectation value in the numerator.

One can easily uplift the entirety of the analysis in Sec. III to this non-Abelian setting. In the Higgs phase, a unit-winding vortex configuration has the form

$$
\begin{aligned}
\Phi(r, \theta) & =v_{\Phi}\left(\begin{array}{c}
f_{+}(r) e^{i(n-1) \theta} \\
f_{-}(r) e^{-i n \theta}
\end{array}\right), \quad \phi_{0}(r, \theta)=v_{0} f_{0}(r) e^{i \theta}, \\
\mathcal{A}_{\theta}(r) & =\frac{a h(r)}{2 \pi r} \frac{\sigma_{3}}{2} .
\end{aligned}
$$

The resulting long-distance energy density, generalizing Eq. (3.8), is

$$
\begin{aligned}
\mathcal{E}(r)= & \frac{v_{\Phi}^{2}}{r^{2}}\left[\left(n-1-\frac{a}{4 \pi}\right)^{2}+\left(-n+\frac{a}{4 \pi}\right)^{2}\right] \\
& +\frac{v_{0}^{2} k^{2}}{r^{2}}+\mathcal{O}\left(r^{-4}\right) .
\end{aligned}
$$

The minimum lies at $a=2 \pi(2 n-1)$, leading to the treelevel result,

$$
\frac{1}{2}\langle\operatorname{tr} \Omega(C)\rangle_{1}=-1
$$

and a phase of $\langle\operatorname{tr} \Omega(C)\rangle_{1}$, at long distance, equal to $\pi$.

\footnotetext{
${ }^{28}$ Alternatively, one might consider writing the Abelian holonomy as the exponential of the magnetic flux through a surface spanning the holonomy contour, and then inserting the definition (B5) of the Abelian flux in terms of the underlying non-Abelian field strength. However, this generalization is undesirable as it converts the original line operator into a surface operator, for which one can no longer argue that the magnitude of the expectation value, in the large $r$ limit, must be independent of the presence of a vortex piercing the surface. With this generalization, the ratio of vortex and ordinary expectation values of the surface operator need not be a pure phase.
}

To see that the phase of $\langle\operatorname{tr} \Omega(C)\rangle_{1}$ must remain at $\pi$ even when quantum fluctuations are taken into account, one can adapt the effective field theory argument at the end of Sec. III B. Integrating out fluctuations generates corrections to the tree-level effective action. The only terms in the effective action that can affect the expectation value of holonomies along contours far from the vortex core are those with exactly two derivatives acting on $\Phi$, because only such operators can affect the $\mathcal{O}\left(1 / r^{2}\right)$ holonomydependent part of the energy density. Given the symmetries of our $S U(2)$ model, all such terms may be written in the form

$$
\begin{aligned}
S_{\text {eff }, S U(2) \text { holonomy }}= & \int d^{3} x\left\{D_{\mu} \Phi^{\dagger} f_{1}\left(\phi_{0}, \zeta, \Phi\right) D^{\mu} \Phi\right. \\
& \left.+\left(D_{\mu} \Phi\right)^{T} i \sigma_{2} f_{2}\left(\phi_{0}, \zeta, \Phi\right) D^{\mu} \Phi\right\}+ \text { H.c. }
\end{aligned}
$$

The functions $f_{1}$ and $f_{2}$, depending on the indicated fields but not their derivatives, transform in the adjoint (or singlet) representation of the $S U(2)$ group. The function $f_{1}$ is invariant under the $U(1)_{\mathrm{G}}$ symmetry and is conjugated by $U_{4}$ under the $\left(\mathbb{Z}_{2}\right)_{\mathrm{F}}$ symmetry (B3) (as $\Phi^{\dagger} \Phi$ or $\Phi \Phi^{\dagger}$ ). The function $f_{2}$ has charge +2 under $U(1)_{\mathrm{G}}$, and transforms as $f_{2} \rightarrow-U_{4} f_{2} U_{4}^{\dagger}$ under $\left(\mathbb{Z}_{2}\right)_{\mathrm{F}}$ [as $\phi_{0} \Phi \Phi^{\dagger} \zeta$ or $\left.i \sigma_{2}\left(\Phi^{\dagger}\right)^{T} \Phi^{\dagger}\right]$.

Just as in Sec. III B, one may verify that both terms, in the presence of a unit-winding vortex, have a minimum at the value $a=2 \pi(2 n-1)$ for the asymptotic coefficient of the gauge field. Therefore, small quantum corrections cannot shift the phase of the holonomy and we learn that

$$
\text { Higgs phase : } O_{\Omega}^{S U(2)}=-1 \text {. }
$$

On the other hand, in the $U(1)_{\mathrm{G}}$-broken confining regime when $m_{\Phi}^{2} \gg g^{4}$, one may reapply the arguments of Sec. III C to show that

$$
U(1)_{\mathrm{G}} \text {-broken confining phase : } O_{\Omega}^{S U(2)}=1 \text {. }
$$

So, as claimed, $O_{\Omega}^{S U(2)}$ serves as an order parameter that distinguishes the $U(1)_{\mathrm{G}}$-broken confining and Higgs phases in this $S U(2)$ gauge theory.

\section{APPENDIX C: $\boldsymbol{U}(1) \times \boldsymbol{U}(1)$ GAUGE THEORY AND TOPOLOGICAL ORDER}

Gauging the global $U(1)_{\mathrm{G}}$ symmetry of our model (2.4), by adding a second dynamical gauge field minimally coupled to the conserved current associated with the $U(1)_{\mathrm{G}}$ symmetry, converts the model into a $U(1) \times$ $U(1)$ gauge theory. This process has the effect of converting the massless Nambu-Goldstone boson associated with spontaneous breaking of the global $U(1)_{\mathrm{G}}$ symmetry into the longitudinal component of a massive gauge field, 
thereby producing a mass gap in the $U(1) \times U(1)$ gauge theory. Superfluid systems (gapless due to global symmetry breaking) and superconducting systems (gapped due to the Meissner effect) are related in precisely this manner.

The $U(1) \times U(1)$ gauge theory produced by gauging the $U(1)_{\mathrm{G}}$ symmetry of our model (2.4) no longer has any continuous global symmetries and is expected to have a nonvanishing mass gap at generic points within its parameter space. This makes it easier to analyze than the gapless models considered in the body of the paper. ${ }^{29}$ The longdistance physics of the $U(1) \times U(1)$ gauge theory can be described by TQFTs at generic points in parameter space. The phase diagram of the $U(1) \times U(1)$ gauge theory produced by gauging the $U(1)_{\mathrm{G}}$ symmetry of our model (2.4) turns out to be very similar to the phase structure of our original model (2.4).

Let $X$ and $Y$ denote the gauge fields associated with each of the $U(1)$ factors of the gauge group, which we henceforth denote as $U(1)_{X} \times U(1)_{Y}$. Let $F_{X}$ and $F_{Y}$ denote the corresponding field strengths, and $e_{X}$ and $e_{Y}$ the gauge couplings of the two different gauge fields. The charge assignments of the scalar fields are

\begin{tabular}{l|ccc} 
& $\phi_{+}$ & $\phi_{-}$ & $\phi_{0}$ \\
\hline$U(1)_{X}$ & +1 & -1 & 0 \\
$U(1)_{Y}$ & -1 & -1 & +2.
\end{tabular}

We assume the standard magnetic flux quantization condition holds for both $F_{X}$ and $F_{Y}$,

$\int_{S^{2}} F_{X}=2 \pi k_{X}, \quad \int_{S^{2}} F_{Y}=2 \pi k_{Y}, \quad k_{X}, k_{Y} \in \mathbb{Z}$,

and assume that finite action monopole instantons preclude the existence of any magnetic $U(1)$ global symmetries. We also assume that the fundamental representation Wilson loop operators $\Omega_{X}=e^{i \int_{C} X}$ and $\Omega_{Y}=e^{i \int_{C} Y}$ are genuine line operators in the sense of Ref. [18].

The action is a simple extension of the original model (2.4),

$$
\begin{aligned}
S= & \int d^{3} x\left[\frac{1}{4 e_{X}^{2}} F_{X}^{2}+\frac{1}{4 e_{Y}^{2}} F_{Y}^{2}+\left|D \phi_{+}\right|^{2}+\left|D \phi_{-}\right|^{2}\right. \\
& +\left|D \phi_{0}\right|^{2}-\epsilon\left(\phi_{+} \phi_{-} \phi_{0}+\text { H.c. }\right)+m_{+}^{2}\left|\phi_{+}\right|^{2}+m_{-}^{2}\left|\phi_{-}\right|^{2} \\
& +m_{0}^{2}\left|\phi_{0}\right|^{2}+\lambda_{+}\left|\phi_{+}\right|^{4}+\lambda_{-}\left|\phi_{-}\right|^{4}+\lambda_{0}\left|\phi_{0}\right|^{4}+\cdots \\
& \left.+V_{\mathrm{m}}\left(\sigma_{X}\right)+V_{\mathrm{m}}\left(\sigma_{Y}\right)\right] .
\end{aligned}
$$

\footnotetext{
${ }^{29}$ We thank Z. Komargodski for urging us to pursue the calculations described in this Appendix.
}

The $V_{\mathrm{m}}\left(\sigma_{X}\right)$ and $V_{\mathrm{m}}\left(\sigma_{Y}\right)$ terms describe the effects of monopole instantons for the $X$ and $Y$ gauge fields, respectively. The cubic $\epsilon$ term ensures that there is no global $U(1)$ symmetry despite the presence of three charged scalar fields and only two gauge bosons.

Our model enjoys Euclidean (or Lorentz) invariance, including reflection and time-reversal symmetry. We do not assume any discrete flavor symmetry permuting the different scalar fields, nor any symmetry interchanging the two $U(1)$ subgroups. ${ }^{30}$ Given the charge assignments $(\mathrm{C} 1)$, the $\mathbb{Z}_{2}$ transformation $(-1,-1) \in U(1)_{X} \times U(1)_{Y}$ acts trivially on all three scalar fields $\phi_{+}, \phi_{-}$, and $\phi_{0}$. Consequently, the theory has a 1-form symmetry, which we denote by $\left(\mathbb{Z}_{2}\right)_{X Y}^{(1)}$, which acts on topologically nontrivial Wilson loops as

$$
\left(\mathbb{Z}_{2}\right)_{X Y}^{(1)}: \Omega_{X} \rightarrow-\Omega_{X}, \quad \Omega_{Y} \rightarrow-\Omega_{Y} .
$$

Now consider the resulting phase diagram. When all three scalars have large positive masses, they can be integrated out resulting in a pure $U(1)_{X} \times U(1)_{Y}$ gauge theory, which has a mass gap and a unique vacuum thanks to the Polyakov mechanism. If only one of the fields $\phi_{ \pm}$is condensed, then there is again a mass gap and a unique vacuum thanks to a combination of the Higgs and Polyakov mechanisms. Other portions of the phase diagram can be mapped out by considering: (i) the regime where $\phi_{0}$ is condensed but the $\phi_{ \pm}$fields are not condensed, and (ii) the regime where all three scalar fields, $\phi_{ \pm}$and $\phi_{0}$, are condensed. So long as the cubic coupling $\epsilon \neq 0$, there is no separate regime where the fields $\phi_{+}$and $\phi_{-}$are condensed, but $\phi_{0}$ is not. Nor is there a regime where, e.g., $\phi_{0}$ and $\phi_{+}$are condensed but $\phi_{-}$is not.

\section{1. $\phi_{0}$ condensed phase}

Suppose that the $\phi_{ \pm}$fields have large positive masses, so that they may be integrated out. The $U(1)_{X}$ gauge field will be gapped, as usual, thanks to the Polyakov mechanism. Condensation of $\phi_{0}$ causes Higgsing of the $U(1)_{Y}$ gauge field, showing that this regime is (generically) gapped. Let us call the regime where only the $\phi_{0}$ field is condensed the $\mathrm{Y}$ regime. Despite the fact that it is gapped, the Y regime has not become completely trivial in the deep infrared because the emergent gauge group at long distances is $\mathbb{Z}_{2}$, and the resultant physics is described by a nontrivial TQFT. $^{31}$

\footnotetext{
${ }^{30}$ A nearly identical model to $(\mathrm{C} 3)$ with a $\mathbb{Z}_{2}$ flavor permutation symmetry was studied in Ref. [106].

${ }^{31}$ See, e.g., Refs. [96,107]. Reference [19] explains that the TQFT associated with a $\mathbb{Z}_{2}$ gauge theory can be viewed as an effective field theory describing a spontaneously broken $\mathbb{Z}_{2}$ 1 -form symmetry. Here, this 1 -form $\mathbb{Z}_{2}$ symmetry acts by flipping the sign of $\Omega_{Y}$.
} 
Before discussing the TQFT description, let us consider the physics of the system through a more direct approach. After integrating out $\phi_{+}$and $\phi_{-}$, the resulting effective action is

$$
\begin{aligned}
S_{\text {eff }}= & \int d^{3} x\left[\frac{1}{4 e_{X}^{2}} F_{X}^{2}+\frac{1}{4 e_{Y}^{2}} F_{Y}^{2}+\left|D \phi_{0}\right|^{2}+V\left(\left|\phi_{0}\right|\right)\right. \\
& +\frac{c_{X}}{m^{2}}\left|\phi_{0}\right|^{2} F_{X}^{2}+\frac{c_{Y}}{m^{2}}\left|\phi_{0}\right|^{2} F_{Y}^{2}+\frac{b_{X}}{m^{2}} \mathcal{S}_{\mu \nu} F_{X}^{\mu \nu} \\
& \left.+\frac{b_{Y}}{m^{2}} \mathcal{S}_{\mu \nu} F_{Y}^{\mu \nu}+\cdots\right]
\end{aligned}
$$

where $m=\min \left(m_{+}, m_{-}\right), \quad D_{\mu} \equiv \partial_{\mu}-2 i Y_{\mu}$, and $\mathcal{S}_{\mu \nu} \equiv$ $\frac{i}{2}\left[\left(D_{\mu} \phi_{0}\right)\left(D_{\nu} \phi_{0}^{\dagger}\right)-\left(D_{\nu} \phi_{0}\right)\left(D_{\mu} \phi_{0}^{\dagger}\right)\right]$. A minimal vortex configuration has the usual form, written as

$$
\phi_{0}(r, \theta)=v_{0} f_{0}(r) e^{i \theta}, \quad Y_{\theta}(r)=\Phi_{Y} h(r) /(2 \pi r),
$$

where $v_{0}$ is the $\phi_{0}$ vacuum expectation value and the radial functions $f_{0}$ and $h$ interpolate between 0 and 1 as $r$ goes from 0 to $\infty$. The asymptotic gauge field coefficient $\Phi_{Y}$ is determined by minimizing the long-distance energy density,

$$
\mathcal{E}(r)=\frac{v_{0}^{2}}{r^{2}}\left(1-\frac{\Phi_{Y}}{\pi}\right)^{2}+\mathcal{O}\left(r^{-4}\right),
$$

which must vanish to prevent a logarithmic IR divergence in the vortex energy, implying that $\Phi_{Y}=\pi$. One can prove that small quantum corrections cannot shift $\Phi_{Y}$ away from this value by using the effective field theory analysis in (3.2). Hence, if $C$ is a large circular contour centered on the vortex, then the holonomy $\Omega_{Y}(C)$ has a phase of $\pi$. More physically, this means that a test particle with a unit charge under $U(1)_{Y}$ picks up a phase of $\pi$ when it moves around a unit-circulation vortex.

Next let us consider the behavior of $X$ holonomies. Consider a test particle with a unit charge under $U(1)_{X}$ and a zero charge under $U(1)_{Y}$. What is the phase acquired by such a test particle when it encircles the $\phi_{0}$ vortex? The answer is not immediately obvious when the coupling $b_{X}$ is nonzero. (A nonzero value for $b_{X}$ may appear in the absence of any flavor permutation symmetry which also flips the sign of $F_{X}$.) In the presence of a winding- $k$ vortex, the antisymmetric tensor $\mathcal{S}_{\mu \nu}$ is nonvanishing with

$$
\mathcal{S}_{r \theta}=\frac{f(r) f^{\prime}(r)}{r}\left[k-\frac{\Phi_{Y}}{\pi} h(r)\right] .
$$

When $k=1$, we know that $\Phi_{Y}=\pi$. When $b_{X} \neq 0$, the source (C8) corresponds to an azimuthal $J_{X}$ current encircling the vortex which, in turn, generates a magnetic field $B_{X}=\frac{1}{2} \epsilon_{i j} F_{X}^{i j}$ localized on the vortex core. Just as in Sec. IIIE, due to confinement, the extent to which this matters depends on the size of the loop with which one probes the system. Consider a spatial disk $D$ with a $\phi_{0}$ vortex at its center and the $\Omega_{X}$ holonomy calculated along the boundary of $D$. When the radius of $D$ is small compared to the string breaking scale, the magnetic flux through $D$ is nonzero, and $O_{\Omega_{X}}=e^{i \Phi}$ with $\Phi \propto b_{X}$. But for holonomies on large contours (with radius $r \gg L_{\mathrm{br}}$ ), string breaking effects remove the sensitivity to $b_{X}$, and we find $O_{\Omega_{X}}=1$. So the expectation values of $X$ holonomies that encircle $\phi_{0}$ vortices on contours $C=\partial D$ are positive in the limit of a large contour radius.

The information about holonomies around vortices is encoded into the TQFT description of the infrared limit of the system. The action for the topological field theory describing the $\mathrm{Y}$ regime, which we denote as $\mathrm{TQFT}_{Y}$, can be written using the $K$-matrix formalism [95,108-113]. In the regime we are considering, $\phi_{0}$ vortex excitations cost finite energy and can be viewed as one type of probe excitation, while test particles with a unit charge under $U(1)_{Y}$ are another. Let $J_{Y}$ denote the conserved $U(1)_{Y}$ current, and $J_{V}$ the topologically conserved vortex number current. These currents couple to two different one-form gauge fields, $a^{i}$, with $i=1$, 2 , each obeying $\int_{M_{2}} d a^{i} \in 2 \pi \mathbb{Z}$ for any closed 2-surface $M_{2}$. Physically, we can identify $a_{\mu}^{1}=Y_{\mu}$, while $a_{\mu}^{2}$ arises in the derivation of the TQFT description as a Lagrange multiplier that enforces the condition that almost everywhere $\epsilon^{\mu \nu \rho} \partial_{\nu} \partial_{\rho} \phi_{0}=0$ while allowing $\oint d x_{\mu} \partial^{\mu} \phi_{0} \in 2 \pi \mathbb{Z}$. Then a description of this $\mathbb{Z}_{2}$ TQFT is provided by the action

$S_{\mathrm{TQFT}_{Y}}=\int d^{3} x\left[\frac{i}{4 \pi}\left(K_{Y}\right)_{i j} \epsilon^{\mu \nu \rho} a_{\mu}^{i} \partial_{\nu} a_{\rho}^{j}+a_{\mu}^{1} J_{Y}^{\mu}+a_{\mu}^{2} J_{V}^{\mu}\right]$,

where the $K$ matrix and its inverse (times $2 \pi$ ) are given by

$$
K_{Y}=\left(\begin{array}{ll}
0 & 2 \\
2 & 0
\end{array}\right), \quad 2 \pi K_{Y}^{-1}=\left(\begin{array}{cc}
0 & \pi \\
\pi & 0
\end{array}\right)
$$

The matrix element $\left(2 \pi K_{Y}^{-1}\right)_{i j}$ gives the phase that an excitation of type $i$ picks up under braiding around one of type $j$. This TQFT has $\left|\operatorname{det} K_{Y}\right|^{g}=4^{g}$ ground states on compact spatial manifolds of genus $g$. Note that the $X$ gauge field does not appear in the TQFT description at all. In this way the TQFT (C9) is implicitly consistent with the above result that all $X$ holonomies have trivial phases in the long-distance limit.

\section{2. $\phi_{0}, \phi_{+}, \phi_{-}$condensed phase}

Now consider the regime where all three scalar fields $\phi_{0}$, $\phi_{+}$, and $\phi_{-}$are condensed, and the $X$ and $Y$ gauge fields are both Higgsed. We will call this the $X Y$ regime. The infrared physics of the $X Y$ regime can be described by a topological field theory which we denote as $\mathrm{TQFT}_{X Y}$. 
Before discussing the TQFT description, it is again useful to explore the physics of vortices directly. Consider the weakly coupled corner of the parameter space of the $X Y$ regime, and suppose that there is a vortex where $\phi_{+}$winds by $2 \pi n_{+}$and $\phi_{-}$winds by $2 \pi n_{-}$on contours encircling the vortex core. Such a field configuration has the form:

$\phi_{+}(r, \theta)=v_{+} f_{+}(r) e^{i n_{+} \theta}, \quad X_{\theta}(r)=\Phi_{X} g(r) /(2 \pi r)$,

$\phi_{-}(r, \theta)=v_{-} f_{-}(r) e^{i n_{-} \theta}, \quad Y_{\theta}(r)=\Phi_{Y} h(r) /(2 \pi r)$,

$\phi_{0}(r, \theta)=v_{0} f_{0}(r) e^{-i\left(n_{+}+n_{-}\right) \theta}$.

The radial functions $f, g$, and $h$ approach 1 as $r \rightarrow \infty$. The long-distance energy density of this vortex configuration is

$$
\begin{aligned}
\mathcal{E}(r)= & \frac{1}{r^{2}}\left[v_{+}^{2}\left(n_{+}-\frac{\Phi_{X}-\Phi_{Y}}{2 \pi}\right)^{2}+v_{-}^{2}\left(n_{-}+\frac{\Phi_{X}+\Phi_{Y}}{2 \pi}\right)^{2}\right. \\
& \left.+v_{0}^{2}\left(n_{+}+n_{-}+\frac{\Phi_{Y}}{\pi}\right)^{2}\right]+\mathcal{O}\left(r^{-4}\right), \quad(\mathrm{C} 12)
\end{aligned}
$$

and, for given values of $n_{+}$and $n_{-}, \mathcal{E}(r)$ is minimized when the $X$ and $Y$ magnetic fluxes $\Phi_{X}, \Phi_{Y}$ are

$$
\Phi_{X}=\left(n_{+}-n_{-}\right) \pi, \quad \Phi_{Y}=-\left(n_{+}+n_{-}\right) \pi .
$$

Since the winding numbers $n_{ \pm}$are integers, these two fluxes are identical modulo $2 \pi$. The vortices with minimal winding and minimal magnetic flux correspond to

\begin{tabular}{l|cccc} 
& $n_{+}$ & $n_{-}$ & $\Phi_{X}$ & $\Phi_{Y}$ \\
\hline$V_{+}$ & 1 & 0 & $\pi$ & $-\pi$ \\
$V_{-}$ & 0 & 1 & $-\pi$ & $-\pi$.
\end{tabular}

The result (C14) shows that test particles with unit charge under $U(1)_{Y}$ pick up a phase of $-\pi$ when encircling either $V_{+}$ or $V_{-}$, while test particles with unit charge under $U(1)_{X}$ pick up a phase of $\pm \pi$ when encircling a $V_{ \pm}$vortex.

The discussion in the body of this paper implies that the $X Y$ and $Y$ regimes cannot be smoothly connected. Both phases contain vortices with flux $\Phi_{Y}=\pi \bmod 2 \pi$, but in the $X Y$ regime any vortices that have $\Phi_{Y}=\pi \bmod 2 \pi$ also have $\Phi_{X}=\pi \bmod 2 \pi$. By comparison, vortices in the $Y$ regime only carry the $Y$ flux. The $X$ flux carried by vortices changes nonanalytically as we go from one regime to the other. We can repeat the logic in Sec. III D to argue that such changes are associated with nonanalyticities in thermodynamic observables, showing that the $Y$ and $X Y$ regimes are distinct phases of matter. The phase transition between the $Y$ and $X Y$ regimes is the parallel of the confinement to Higgs phase transition discussed in the main part of this paper.

We now consider the TQFT description of the longdistance physics of the XY regime. Given the charge assignments $(\mathrm{C} 1)$, the unbroken part of the gauge group in the $X Y$ regime is generated by the $\mathbb{Z}_{2}$ transformation $(-1,-1) \in U(1)_{X} \times U(1)_{Y}$, and the 1-form symmetry $\left(\mathbb{Z}_{2}\right)_{X Y}^{(1)}$, acting as shown in Eq. (C4), is spontaneously broken. We can derive the appropriate TQFT describing this regime directly from the original model (C3) in the weakly coupled corner of parameter space of the $X Y$ regime. By virtue of being topological, the resulting effective action will furnish a valid description of the physics even away from the weak coupling limit. We follow a procedure similar to that in Sec. 3.3 of Ref. [96]. In the weak coupling and long distance limits, we can freeze the moduli of the scalar fields to their vacuum expectation values because all physical fluctuation modes around the expectation values are gapped and can be integrated out. Let us denote the phases of the three scalar fields by $\varphi_{0}, \varphi_{+}$, and $\varphi_{-}$. Since we are interested in the low energy form of the effective action, we note that minimizing the cubic $\epsilon$ term in model (C3) implies that $\varphi_{0}+\varphi_{+}+\varphi_{-}=0$. With all this taken into account, the relevant part of the effective action becomes just

$$
\begin{aligned}
\mathcal{L}_{\text {Stückelberg }}= & v_{+}^{2}\left(\partial_{\mu} \varphi_{+}-X_{\mu}+Y_{\mu}\right)^{2} \\
& +v_{-}^{2}\left(\partial_{\mu} \varphi_{-}+X_{\mu}+Y_{\mu}\right)^{2} \\
& +v_{0}^{2}\left(\partial_{\mu} \varphi_{+}+\partial_{\mu} \varphi_{-}+2 Y_{\mu}\right)^{2},
\end{aligned}
$$

where $v_{0}, v_{+}$, and $v_{-}$are the magnitudes of the expectation values of $\phi_{0}, \phi_{+}$, and $\phi_{-}$, respectively. We have dropped the Maxwell terms because we are interested in length scales which are large compared to $1 / e_{X}^{2}$ and $1 / e_{Y}^{2}$. The long distance TQFT is obtained by dualizing $\varphi_{+}$and $\varphi_{-}$ and taking the low energy limit $v_{0}, v_{+}, v_{-} \rightarrow \infty$. To this end, we introduce one-form Lagrange multiplier fields $a^{+}$ and $a^{-}$satisfying $\int_{M_{2}} d a^{ \pm} \in 2 \pi \mathbb{Z}$ for any closed 2-manifold $M_{2}$. Then the Lagrangian,

$\mathcal{L}_{\text {dual }}=\mathcal{L}_{\text {Stückelberg }}+\frac{i}{2 \pi} \epsilon^{\mu \nu \alpha} a_{\mu}^{+} \partial_{\nu} \partial_{\alpha} \varphi_{+}+\frac{i}{2 \pi} \epsilon^{\mu \nu \alpha} a_{\mu}^{-} \partial_{\nu} \partial_{\alpha} \varphi_{-}$,

enforces $\epsilon^{\mu \nu \rho} \partial_{\nu} \partial_{\rho} \varphi_{ \pm}=0$ almost everywhere while allowing $\oint d x_{\mu} \partial^{\mu} \varphi_{ \pm} \in 2 \pi \mathbb{Z}$. Using the resulting equations of motion one finds

$\partial^{\mu} \varphi_{ \pm}= \pm X^{\mu}-Y^{\mu}-\frac{i}{4 \pi} \frac{\left(v_{\mp}^{2}+v_{0}^{2}\right) \epsilon^{\mu \nu \rho} \partial_{\nu} a_{\rho}^{ \pm}-v_{0}^{2} \epsilon^{\mu \nu \rho} \partial_{\nu} a_{\rho}^{\mp}}{v_{0}^{2} v_{+}^{2}+v_{0}^{2} v_{-}^{2}+v_{+}^{2} v_{-}^{2}}$

and 


$$
\begin{aligned}
\mathcal{L}_{\text {dual }}= & \frac{i}{2 \pi} \epsilon^{\mu \nu \rho}\left[\left(X_{\mu}-Y_{\mu}\right) \partial_{\nu} a_{\rho}^{+}\right. \\
& \left.-\left(X_{\mu}+Y_{\mu}\right) \partial_{\nu} a_{\rho}^{-}\right]+\mathcal{O}\left(v_{i}^{-2}\right)
\end{aligned}
$$

This Lagrangian (when $v_{i} \rightarrow \infty$ ) describes a topological field theory, TQFT $T_{X Y}$. To write the result in a more useful form, denote the set of gauge fields by $\left\{a_{\mu}^{i}\right\}=$ $\left\{X_{\mu}, Y_{\mu}, a_{\mu}^{+}, a_{\mu}^{-}\right\}$. We also introduce a set of currents $\left\{J_{\mu}^{i}\right\}$, which are, respectively, the $U(1)_{X}$ and $U(1)_{Y}$ Noether currents and the topological vortex number currents associated with $\phi_{+}$and $\phi_{-}$vortices. Then the action for $\mathrm{TQFT}_{X Y}$ can be written as

$$
S_{\mathrm{TQFT}_{X Y}}=\int d^{3} x\left[\frac{i}{4 \pi}\left(K_{X Y}\right)_{i j} \epsilon^{\mu \nu \rho} a_{\mu}^{i} \partial_{\nu} a_{\rho}^{j}+a_{\mu}^{i}\left(J^{\mu}\right)_{i}\right],
$$

with $K$ matrix (and its inverse)

$$
\begin{aligned}
K_{X Y} & =\left(\begin{array}{cccc}
0 & 0 & 1 & -1 \\
0 & 0 & -1 & -1 \\
1 & -1 & 0 & 0 \\
-1 & -1 & 0 & 0
\end{array}\right), \\
2 \pi K_{X Y}^{-1} & =\left(\begin{array}{cccc}
0 & 0 & \pi & -\pi \\
0 & 0 & -\pi & -\pi \\
\pi & -\pi & 0 & 0 \\
-\pi & -\pi & 0 & 0
\end{array}\right) .
\end{aligned}
$$

This TQFT has $\left|\operatorname{det} K_{X Y}\right|^{g}=4^{g}$ ground states on spatial manifolds of genus $g$ in accordance with expectations from the spontaneously broken $\left(\mathbb{Z}_{2}\right)_{X Y}^{(1)}$ symmetry.

It is possible to find an interesting relation between $\mathrm{TQFT}_{Y}$ and TQFT $X Y$. Suppose we add a new spectator field $\chi$ which has charge +1 under a new gauge field $Z_{\mu}$, obeying standard flux quantization conditions, and assume that the microscopic theory admits finite action $Z$ monopole events. We further suppose that $\chi$ is condensed in the $Y$ regime and has a large positive mass squared in the $X Y$ regime. Then the $K$ matrix of $\mathrm{TQFT}_{X Y}$ remains unchanged, but the $K$ matrix of the $Y$ regime is enlarged and becomes

$$
\tilde{K}_{Y}=\left(\begin{array}{llll}
0 & 2 & 0 & 0 \\
2 & 0 & 0 & 0 \\
0 & 0 & 0 & 1 \\
0 & 0 & 1 & 0
\end{array}\right) .
$$

The action of the $Y$ regime is now $\int d^{3} x\left[\frac{i}{4 \pi} \epsilon^{\mu \nu \rho} a_{\mu}^{i}\left(\tilde{K}_{Y}\right)_{i j} \times\right.$ $\left.\partial_{\nu} a_{\rho}^{j}+a_{\mu}^{i} J_{i}^{\mu}\right]$, with the set of gauge fields $\left\{a_{\mu}^{i}\right\}=$ $\left\{Y_{\mu}, a_{\mu}^{0}, Z_{\mu}, a_{\mu}^{\chi}\right\}$. Here $a_{\mu}^{0}$ and $a_{\mu}^{\chi}$ are auxiliary gauge fields that couple to the $\phi_{0}$ and $\chi$ vortex currents, respectively.
Then one can verify the congruence relation $G^{T} K_{X Y} G=$ $\tilde{K}_{Y}$ where $G \in G L(4, \mathbb{Z})$ is the matrix

$$
G=\left(\begin{array}{cccc}
-1 & 0 & 0 & 0 \\
1 & 0 & -1 & 0 \\
0 & -1 & 0 & 0 \\
0 & 1 & 0 & 1
\end{array}\right)
$$

This shows that the extended set of gauge fields in the $Y$ regime are related to those in the $X Y$ regime by the change of basis $a_{Y}=G a_{X Y}$, or explicitly

$$
\left(\begin{array}{c}
Y \\
a^{0} \\
Z \\
a^{\chi}
\end{array}\right) \rightarrow\left(\begin{array}{c}
-X \\
-a^{+} \\
-X-Y \\
a^{+}+a^{-}
\end{array}\right) .
$$

We emphasize that the existence of the relation (C23) does not contradict our assertion above that the $Y$ and $X Y$ regimes cannot be smoothly connected and must be separated by a phase boundary. ${ }^{32}$

\section{Phase transitions and ungauging limits}

Having just seen the $U(1) \times U(1)$ gauge theory has distinct gapped phases which are necessarily separated by phase transitions, in a completely parallel fashion with what we inferred by direct calculations in the original $U(1)$ gauge theory with gapless phases, it is natural to ask whether our direct study in the original $U(1)$ gauge theory was really necessary. In other words, can one presume that distinct phases present after one weakly gauges a continuous global symmetry survive the limit of sending the coupling of the artificially introduced gauge field back to zero? Alternatively, if two regimes can be smoothly connected in a given theory with a dynamical gauge field, does this necessarily remain true in the ungauging limit? We argue that the answer to both of these questions is no.

For systems with discrete symmetry groups, it has been established that phase transitions detected by changes in particle-vortex statistics in gauged models imply phase transitions in the parallel ungauged models (see, e.g., Refs. [114-116]). It may be tempting to assume that the same should be true with continuous symmetries, and in some simple examples this parallel between phases in gauged and ungauged models does hold. For instance, the phase structure of a theory of a single parity-invariant complex scalar field in three spacetime dimensions with a

\footnotetext{
${ }^{32}$ For an analogous example, recall that the high and low temperature regimes of the 2D Ising model on a square lattice are related by Kramers-Wannier duality. Nevertheless, the high and low temperature regimes are distinct phases separated by a phase transition at the self-dual point.
} 
$U(1)$ global symmetry does not change when the $U(1)$ symmetry is gauged (provided there are no monopoles), thanks to particle-vortex duality $[117,118]$. Naively one might take this example as part of a general pattern and guess that phase transitions in the $U(1)_{X} \times U(1)_{Y}$ gauge theory necessarily imply phase transitions in $U(1)_{X}$ gauge theory obtained via the "ungauging" limit $e_{Y} \rightarrow 0$.

But it is not correct to presume, in general, that the phase structure of a theory with a continuous global symmetry must be identical to that of the gauged version of the theory. It is quite possible that as the gauge coupling is sent to zero, a phase boundary appears between two regimes which were smoothly connected in the gauged model. Similarly, nonanalyticities present in thermodynamic functions of the gauged model may disappear when the gauge coupling is sent to zero. ${ }^{33}$

It is easy to find examples illustrating the above scenarios. First, consider again a compact $U(1)$ gauge theory with a single complex scalar with charge +1 in three spacetime dimensions. Unlike the discussion above, suppose we specify a UV completion of the theory that does admit finite-action monopole-instanton events with minimal magnetic flux. Then the Higgs and confining regimes of the gauge theory are smoothly connected. However, if we ungauge the $U(1)$ symmetry, we are left with the $X Y$ model in 3D, which has $U(1)$ global symmetry. The $U(1)$ symmetry broken and unbroken regimes of the $X Y$ theory are the limits of the Higgs and confining regimes of the gauge theory. But these regimes are separated by a phase boundary in the 3D $X Y$ model.

It is also possible for phase boundaries of a gauge theory to disappear in the ungauging limit. To see an example of this consider four free massless Dirac fermions in 4D spacetime. Such a system has a global symmetry that includes $S U(4)_{L} \times S U(4)_{R}$. If we gauge the vectorlike $S U(2)_{V}$ subgroup of this symmetry, introducing a gauge

\footnotetext{
${ }^{33}$ This subtlety does not arise in the case of gauged discrete symmetries studied in Refs. [114-116], because gauging discrete symmetries introduces neither local degrees of freedom nor continuous coupling constants.
}

coupling $g$, we obtain two-color two-flavor massless QCD. It has a $S U(2)_{L} \times S U(2)_{R}$ global symmetry, and an $S U(2)_{A}$-breaking phase transition as a function of temperature. The critical temperature has a nonperturbative dependence on $g$ due to dimensional transmutation. The phase transition exists for any nonzero value of the $S U(2)$ gauge coupling $g$, but disappears at $g=0$ where the theory becomes free. Another example is given by $N_{c} N_{f}$ free massless Dirac fermions in 4D spacetime when one gauges an $S U\left(N_{c}\right)$ subgroup of the vectorlike global symmetry, yielding massless QCD with $N_{f}$ massless quark flavors. In the large $N_{c}$ limit with $N_{f} / N_{c}$ and $g^{2} N_{c}$ fixed, the model is known to go through at least two quantum phase transitions as a function of $N_{f} / N_{c}$ when $g^{2} N_{c}$ is fixed as a nonzero (see, e.g., Refs. $[119,120]$ ). But there are no such phase transitions at $g=0$.

Similar concerns apply to the continuous Abelian gauge theories we focused on in this paper. In the $U(1)_{X} \times U(1)_{Y}$ gauge theory (C3), one can infer the existence of phase transitions from the behavior of gauge field holonomies whose values become quantized on large distance scales. Holonomy quantization only holds on distance scales large compared to all relevant length scales. If one is interested in the behavior of the system with generic values of its physical parameters, then this is not a problem. But the limit of vanishing gauge coupling, $e_{Y} \rightarrow 0$, is a highly nongeneric limit. In this limit, in Higgs phases of the theory, the physical mass of a gauge boson goes to zero. In other words, the gauge boson Compton wavelength diverges, and consequently the length scale on which holonomy quantization holds also diverges. More formally, there is nonuniformity between the limit $e_{Y} \rightarrow 0$ and the large distance limit implicit in defining the vortex holonomy. And this means that one cannot simply infer the phase structure of the theory exactly at $e_{Y}=0$ from an analysis with $e_{Y}>0$. In particular, the only way to demonstrate that nonanalyticities inferred by analysis of the gauged, gapped theory survive to become corresponding observable nonanalyticities in the original gapless theory is to roll up one's sleeves and examine the gapless theory of interest. This, of course, was the main goal of this paper.
[1] G. 't Hooft, Which topological features of a gauge theory can be responsible for permanent confinement?, NATO Sci. Ser. B 59, 117 (1980).

[2] K. Osterwalder and E. Seiler, Gauge field theories on the lattice, Ann. Phys. (N.Y.) 110, 440 (1978).

[3] E. H. Fradkin and S. H. Shenker, Phase diagrams of lattice gauge theories with Higgs fields, Phys. Rev. D 19, 3682 (1979).
[4] T. Banks and E. Rabinovici, Finite temperature behavior of the lattice Abelian Higgs model, Nucl. Phys. B160, 349 (1979).

[5] K. Fredenhagen and M. Marcu, A Confinement Criterion for QCD with Dynamical Quarks, Phys. Rev. Lett. 56, 223 (1986).

[6] J. Greensite and K. Matsuyama, Confinement criterion for gauge theories with matter fields, Phys. Rev. D 96, 094510 (2017). 
[7] J. Greensite and K. Matsuyama, What symmetry is actually broken in the Higgs phase of a gauge-Higgs theory?, Phys. Rev. D 98, 074504 (2018).

[8] J. Greensite and K. Matsuyama, Higgs phase as a spin glass and the transition between varieties of confinement, Phys. Rev. D 101, 054508 (2020).

[9] F. Wegner, Duality in generalized Ising models and phase transitions without local order parameters, J. Math. Phys. (N.Y.) 12, 2259 (1971).

[10] X. G. Wen, Vacuum degeneracy of chiral spin states in compactified space, Phys. Rev. B 40, 7387 (1989).

[11] X. G. Wen, Topological order in rigid states, Int. J. Mod. Phys. B 04, 239 (1990).

[12] X.-G. Wen, Topological order: From long-range entangled quantum matter to an unification of light and electrons, ISRN Cond. Matt. Phys. 2013, 198710 (2013).

[13] S. Sachdev and K. Park, Ground states of quantum antiferromagnets in two dimensions, Ann. Phys. (N.Y.) 298, 58 (2002).

[14] A. Kitaev, Anyons in an exactly solved model and beyond, Ann. Phys. (Amsterdam) 321, 2 (2006).

[15] S. Sachdev, Topological order, emergent gauge fields, and Fermi surface reconstruction, Rep. Prog. Phys. 82, 014001 (2019).

[16] S. Gukov and A. Kapustin, Topological quantum field theory, nonlocal operators, and gapped phases of gauge theories, arXiv:1307.4793.

[17] A. Kapustin and R. Thorngren, Higher symmetry and gapped phases of gauge theories, arXiv:1309.4721.

[18] A. Kapustin and N. Seiberg, Coupling a QFT to a TQFT and duality, J. High Energy Phys. 04 (2014) 001.

[19] D. Gaiotto, A. Kapustin, N. Seiberg, and B. Willett, Generalized global symmetries, J. High Energy Phys. 02 (2015) 172.

[20] M. A. Metlitski and R. Thorngren, Intrinsic and emergent anomalies at deconfined critical points, Phys. Rev. B 98, 085140 (2018).

[21] E. Lake, Higher-form symmetries and spontaneous symmetry breaking, arXiv:1802.07747.

[22] X.-G. Wen, Emergent anomalous higher symmetries from topological order and from dynamical electromagnetic field in condensed matter systems, Phys. Rev. B 99, 205139 (2019).

[23] A. Cherman, S. Sen, and L. G. Yaffe, Anyonic particlevortex statistics and the nature of dense quark matter, Phys. Rev. D 100, 034015 (2019).

[24] T. Schäfer and F. Wilczek, Continuity of Quark and Hadron Matter, Phys. Rev. Lett. 82, 3956 (1999).

[25] A. M. Polyakov, Quark confinement and topology of gauge groups, Nucl. Phys. B120, 429 (1977).

[26] M. G. Alford, A. Schmitt, K. Rajagopal, and T. Schäfer, Color superconductivity in dense quark matter, Rev. Mod. Phys. 80, 1455 (2008).

[27] C. Vafa and E. Witten, Restrictions on symmetry breaking in vector-like gauge theories, Nucl. Phys. B234, 173 (1984).

[28] D. Gaiotto, A. Kapustin, Z. Komargodski, and N. Seiberg, Theta, time reversal, and temperature, J. High Energy Phys. 05 (2017) 091.
[29] M. Hindmarsh and T. W. B. Kibble, Beads on Strings, Phys. Rev. Lett. 55, 2398 (1985).

[30] D. Tong, Monopoles in the higgs phase, Phys. Rev. D 69 , 065003 (2004).

[31] M. Shifman and A. Yung, NonAbelian string junctions as confined monopoles, Phys. Rev. D 70, 045004 (2004).

[32] A. Hanany and D. Tong, Vortex strings and fourdimensional gauge dynamics, J. High Energy Phys. 04 (2004) 066.

[33] M. Eto, M. Nitta, and N. Yamamoto, Instabilities of NonAbelian Vortices in Dense QCD, Phys. Rev. Lett. 104, 161601 (2010).

[34] M. Eto and M. Nitta, Color magnetic flux tubes in dense QCD, Phys. Rev. D 80, 125007 (2009).

[35] M. Cipriani, D. Dorigoni, S. B. Gudnason, K. Konishi, and A. Michelini, Non-Abelian monopole-vortex complex, Phys. Rev. D 84, 045024 (2011).

[36] A. Gorsky, M. Shifman, and A. Yung, Confined magnetic monopoles in dense QCD, Phys. Rev. D 83, 085027 (2011).

[37] C. Chatterjee and M. Nitta, Aharonov-Bohm defects, Phys. Rev. D 101, 085002 (2020).

[38] S. R. Coleman, R. Jackiw, and L. Susskind, Charge shielding and quark confinement in the massive Schwinger model, Ann. Phys. (N.Y.) 93, 267 (1975).

[39] S. R. Coleman, More about the massive Schwinger model, Ann. Phys. (N.Y.) 101, 239 (1976).

[40] E. Witten, $\theta$ Vacua in two-dimensional quantum chromodynamics, Nuovo Cimento A 51, 325 (1979).

[41] M. M. Anber and E. Poppitz, Anomaly matching, (axial) Schwinger models, and high-T super Yang-Mills domain walls, J. High Energy Phys. 09 (2018) 076.

[42] M. M. Anber and E. Poppitz, Domain walls in high-T SU(N) super Yang-Mills theory and QCD(adj), J. High Energy Phys. 05 (2019) 151.

[43] A. Armoni and S. Sugimoto, Vacuum structure of charge k two-dimensional QED and dynamics of an anti D-string near an O1-plane, J. High Energy Phys. 03 (2019) 175.

[44] T. Misumi, Y. Tanizaki, and M. Ünsal, Fractional $\theta$ angle, 't Hooft anomaly, and quantum instantons in charge- $q$ multi-flavor Schwinger model, J. High Energy Phys. 07 (2019) 018.

[45] S. R. Coleman and E. J. Weinberg, Radiative corrections as the origin of spontaneous symmetry breaking, Phys. Rev. D 7, 1888 (1973).

[46] M. Unsal and L. G. Yaffe, Center-stabilized Yang-Mills theory: Confinement and large $N$ volume independence, Phys. Rev. D 78, 065035 (2008).

[47] K. Aitken, A. Cherman, E. Poppitz, and L. G. Yaffe, QCD on a small circle, Phys. Rev. D 96, 096022 (2017).

[48] G. Baym, T. Hatsuda, T. Kojo, P. D. Powell, Y. Song, and T. Takatsuka, From hadrons to quarks in neutron stars: A review, Rep. Prog. Phys. 81, 056902 (2018).

[49] D. Rischke, D. Son, and M. A. Stephanov, Asymptotic Deconfinement in High Density QCD, Phys. Rev. Lett. 87, 062001 (2001).

[50] D. Dean and M. Hjorth-Jensen, Pairing in nuclear systems: From neutron stars to finite nuclei, Rev. Mod. Phys. 75, 607 (2003). 
[51] S. Gandolfi, A. Gezerlis, and J. Carlson, Neutron matter from low to high density, Annu. Rev. Nucl. Part. Sci. 65, 303 (2015).

[52] J. Greensite, Confinement from center vortices: A review of old and new results, EPJ Web Conf. 137, 01009 (2017).

[53] K. Rajagopal and F. Wilczek, The condensed matter physics of QCD, in At the Frontier of Particle Physics. Handbook of $Q C D$, edited by M. Shifman and B. Ioffe (World Scientific, Singapore, 2000), Vols. 1-3, pp. 2061-2151.

[54] Z. Wan and J. Wang, Higher anomalies, higher symmetries, and Cobordisms III: QCD matter phases anew, Nucl. Phys. B957, 115016 (2020).

[55] L.-M. Lin, K. Cheng, M.-C. Chu, and W.-M. Suen, Gravitational waves from phase-transition induced collapse of neutron stars, Astrophys. J. 639, 382 (2006).

[56] I. Sagert, T. Fischer, M. Hempel, G. Pagliara, J. SchaffnerBielich, A. Mezzacappa, F.-K. Thielemann, and M. Liebendorfer, Signals of the QCD Phase Transition in Core-Collapse Supernovae, Phys. Rev. Lett. 102, 081101 (2009).

[57] J. M. Lattimer and M. Prakash, The equation of state of hot, dense matter and neutron stars, Phys. Rep. 621, 127 (2016).

[58] M. G. Alford and S. Han, Characteristics of hybrid compact stars with a sharp hadron-quark interface, Eur. Phys. J. A 52, 62 (2016).

[59] S. Han and A. W. Steiner, Tidal deformability with sharp phase transitions in (binary) neutron stars, Phys. Rev. D 99, 083014 (2019).

[60] E. R. Most, L. J. Papenfort, V. Dexheimer, M. Hanauske, S. Schramm, H. Stöcker, and L. Rezzolla, Signatures of Quark-Hadron Phase Transitions in General-Relativistic Neutron-Star Mergers, Phys. Rev. Lett. 122, 061101 (2019).

[61] L. McLerran and S. Reddy, Quarkyonic Matter and Neutron Stars, Phys. Rev. Lett. 122, 122701 (2019).

[62] A. Bauswein, N.-U.F. Bastian, D. B. Blaschke, K. Chatziioannou, J. A. Clark, T. Fischer, and M. Oertel, Identifying a First-Order Phase Transition in Neutron Star Mergers Through Gravitational Waves, Phys. Rev. Lett. 122, 061102 (2019).

[63] J.-E. Christian, A. Zacchi, and J. Schaffner-Bielich, Signals in the tidal deformability for phase transitions in compact stars with constraints from GW170817, Phys. Rev. D 99, 023009 (2019).

[64] C.-J. Xia, T. Maruyama, N. Yasutake, and T. Tatsumi, Constraining quark-hadron interface tension in the multimessenger era, Phys. Rev. D 99, 103017 (2019).

[65] S. Gandolfi, J. Lippuner, A. W. Steiner, I. Tews, X. Du, and M. Al-Mamun, From the microscopic to the macroscopic world: from nucleons to neutron stars, J. Phys. G $\mathbf{4 6}$, 103001 (2019).

[66] H.-Y. Chen, P. M. Chesler, and A. Loeb, Searching for exotic cores with binary neutron star inspirals, Astrophys. J. Lett. 893, L4 (2020).

[67] M. G. Alford, S. Han, and K. Schwenzer, Signatures for quark matter from multi-messenger observations, J. Phys. G 46, 114001 (2019).
[68] S. Han, M. A. A. Mamun, S. Lalit, C. Constantinou, and M. Prakash, Treating quarks within neutron stars, Phys. Rev. D 100, 103022 (2019).

[69] J.-E. Christian and J. Schaffner-Bielich, Twin stars and the stiffness of the nuclear equation of state: Ruling out strong phase transitions below $1.7 n_{0}$ with the new NICER radius measurements, Astrophys. J. Lett. 894, L8 (2020).

[70] K. Chatziioannou and S. Han, Studying strong phase transitions in neutron stars with gravitational waves, Phys. Rev. D 101, 044019 (2020).

[71] E. Annala, T. Gorda, A. Kurkela, J. Nättilä, and A. Vuorinen, Evidence for quark-matter cores in massive neutron stars, Nat. Phys. 16, 907 (2020).

[72] P. M. Chesler, N. Jokela, A. Loeb, and A. Vuorinen, Finitetemperature equations of state for neutron star mergers, Phys. Rev. D 100, 066027 (2019).

[73] T. Fischer, M.-R. Wu, B. Wehmeyer, N.-U. F. Bastian, G. Martínez-Pinedo, and F.-K. Thielemann, Core-collapse supernova explosions driven by the hadron-quark phase transition as a rare $r$-process site, Astrophys. J. 894, 9 (2020).

[74] S. Zha, E. P. O'Connor, M.-c. Chu, L.-M. Lin, and S. M. Couch, Gravitational-Wave Signature of a First-Order Quantum Chromodynamics Phase Transition in CoreCollapse Supernovae, Phys. Rev. Lett. 125, 051102 (2020).

[75] T. Hatsuda, M. Tachibana, N. Yamamoto, and G. Baym, New Critical Point Induced by the Axial Anomaly in Dense QCD, Phys. Rev. Lett. 97, 122001 (2006).

[76] L. McLerran and R. D. Pisarski, Phases of cold, dense quarks at large N(c), Nucl. Phys. A796, 83 (2007).

[77] N. Yamamoto, M. Tachibana, T. Hatsuda, and G. Baym, Phase structure, collective modes, and the axial anomaly in dense QCD, Phys. Rev. D 76, 074001 (2007).

[78] G. Baym, S. Furusawa, T. Hatsuda, T. Kojo, and H. Togashi, New neutron star equation of state with quarkhadron crossover, Astrophys. J. 885, 42 (2019).

[79] K. Nishimura and N. Yamamoto, Topological term, QCD anomaly, and the eta' chiral soliton lattice in rotating baryonic matter, J. High Energy Phys. 07 (2020) 196.

[80] Y. Hirono and Y. Tanizaki, Quark-Hadron Continuity beyond the Ginzburg-Landau Paradigm, Phys. Rev. Lett. 122, 212001 (2019).

[81] K. B. Fadafan, F. Kazemian, and A. Schmitt, Towards a holographic quark-hadron continuity, J. High Energy Phys. 03 (2019) 183.

[82] A. Schmitt, S. Stetina, and M. Tachibana, GinzburgLandau phase diagram for dense matter with axial anomaly, strange quark mass, and meson condensation, Phys. Rev. D 83, 045008 (2011).

[83] M. Buballa, NJL model analysis of quark matter at large density, habilitation thesis, 2005.

[84] K. Fukushima and C. Sasaki, The phase diagram of nuclear and quark matter at high baryon density, Prog. Part. Nucl. Phys. 72, 99 (2013).

[85] K. Fukushima and T. Kojo, The quarkyonic star, Astrophys. J. 817, 180 (2016).

[86] T. Schäfer and F. Wilczek, Quark description of hadronic phases, Phys. Rev. D 60, 074014 (1999). 
[87] T. Schäfer, Quark hadron continuity in QCD with one flavor, Phys. Rev. D 62, 094007 (2000).

[88] K. Masuda, T. Hatsuda, and T. Takatsuka, Hadron-quark crossover and massive hybrid stars, Prog. Theor. Exp. Phys. 2013, 073 D01 (2013).

[89] N. Kovensky and A. Schmitt, Holographic quarkyonic matter, J. High Energy Phys. 09 (2020) 112.

[90] M. Cipriani, W. Vinci, and M. Nitta, Colorful boojums at the interface of a color superconductor, Phys. Rev. D 86, 121704 (2012).

[91] C. Chatterjee and M. Nitta, Aharonov-Bohm phase in high density quark matter, Phys. Rev. D 93, 065050 (2016).

[92] C. Chatterjee, M. Nitta, and S. Yasui, Quark-hadron continuity under rotation: Vortex continuity or boojum?, Phys. Rev. D 99, 034001 (2019).

[93] Y. Hirono and Y. Tanizaki, Effective gauge theories of superfluidity with topological order, J. High Energy Phys. 07 (2019) 062.

[94] M. G. Alford, G. Baym, K. Fukushima, T. Hatsuda, and M. Tachibana, Continuity of vortices from the hadronic to the color-flavor locked phase in dense matter, Phys. Rev. D 99, 036004 (2019).

[95] X.-G. Wen, Topological orders and edge excitations in fractional quantum hall states, Adv. Phys. 44, 405 (1995).

[96] T. H. Hansson, V. Oganesyan, and S. L. Sondhi, Superconductors are topologically ordered, Ann. Phys. (Amsterdam) 313, 497 (2004).

[97] R. Auzzi, S. Bolognesi, J. Evslin, K. Konishi, and A. Yung, NonAbelian superconductors: Vortices and confinement in $N=2$ SQCD, Nucl. Phys. B673, 187 (2003).

[98] M. Eto, M. Nitta, and N. Yamamoto, Confined monopoles induced by quantum effects in dense QCD, Phys. Rev. D 83, 085005 (2011).

[99] M. Campostrini, P. Rossi, and E. Vicari, Monte Carlo simulation of $\mathrm{CP} * *(\mathrm{~N}-1)$ models, Phys. Rev. D 46, 2647 (1992).

[100] C. Bonati, A. Pelissetto, and E. Vicari, Two-dimensional multicomponent Abelian-Higgs lattice models, Phys. Rev. D 101, 034511 (2020).

[101] G. 't Hooft, Magnetic monopoles in unified gauge theories, Nucl. Phys. B79, 276 (1974).

[102] A. M. Polyakov, Pis'ma Zh. Eksp. Teor. Fiz. 20, 430 (1974) [Particle spectrum in the quantum field theory, JETP Lett. 20, 194 (1974)].

[103] E. B. Bogomolny, Stability of classical solutions, Yad. Fiz. 24, 861 (1976) [Sov. J. Nucl. Phys. 24, 449 (1976)].

[104] M. K. Prasad and C. M. Sommerfield, An Exact Classical Solution for the 't Hooft Monopole and the Julia-Zee Dyon, Phys. Rev. Lett. 35, 760 (1975).
[105] T. W. Kirkman and C. K. Zachos, Asymptotic analysis of the monopole structure, Phys. Rev. D 24, 999 (1981).

[106] S. M. Carroll and M. Trodden, Dirichlet topological defects, Phys. Rev. D 57, 5189 (1998).

[107] J. M. Maldacena, G. W. Moore, and N. Seiberg, D-brane charges in five-brane backgrounds, J. High Energy Phys. 10 (2001) 005.

[108] N. Read, Excitation Structure of the Hierarchy Scheme in the Fractional Quantum Hall Effect, Phys. Rev. Lett. 65, 1502 (1990).

[109] B. Blok and X. G. Wen, Effective theories of the fractional quantum hall effect: Hierarchy construction, Phys. Rev. B 42, 8145 (1990).

[110] X. G. Wen and A. Zee, Classification of abelian quantum hall states and matrix formulation of topological fluids, Phys. Rev. B 46, 2290 (1992).

[111] Y.-M. Lu and A. Vishwanath, Theory and classification of interacting 'integer' topological phases in two dimensions: A Chern-Simons approach, Phys. Rev. B 86, 125119 (2012); Erratum, Phys. Rev. B 89, 199903 (2014).

[112] Y.-M. Lu and A. Vishwanath, Classification and properties of symmetry-enriched topological phases: Chern-Simons approach with applications to $\mathrm{Z}_{2}$ spin liquids, Phys. Rev. B 93, 155121 (2016).

[113] D. Delmastro and J. Gomis, Symmetries of Abelian ChernSimons theories and arithmetic, arXiv:1904.12884.

[114] M. Levin and Z.-C. Gu, Braiding statistics approach to symmetry-protected topological phases, Phys. Rev. B 86, 115109 (2012).

[115] C. Wang and M. Levin, Braiding Statistics of Loop Excitations in Three Dimensions, Phys. Rev. Lett. 113, 080403 (2014).

[116] C. Wang and M. Levin, Topological invariants for gauge theories and symmetry-protected topological phases, Phys. Rev. B 91, 165119 (2015).

[117] M. E. Peskin, Mandelstam 't Hooft duality in Abelian lattice models, Ann. Phys. (N.Y.) 113, 122 (1978).

[118] C. Dasgupta and B. I. Halperin, Phase Transition in a Lattice Model of Superconductivity, Phys. Rev. Lett. 47, 1556 (1981).

[119] W. E. Caswell, Asymptotic Behavior of Nonabelian Gauge Theories to Two Loop Order, Phys. Rev. Lett. 33, 244 (1974).

[120] T. Banks and A. Zaks, On the phase structure of vector-like gauge theories with massless fermions, Nucl. Phys. B196, 189 (1982). 